\title{
Brachymelecta Linsley, 1939, previously the rarest North American bee genus, was described from an aberrant specimen and is the senior synonym for Xeromelecta Linsley, 1939
}

\author{
Thomas M. ONUFERKO $^{{ }^{\circledR}{ }^{1, *}, \text { Laurence PACKER }^{\circledR}{ }^{2}}$ \& Julio A. GENARO ${ }^{\oplus_{3}}$ \\ ${ }^{1}$ Beaty Centre for Species Discovery, Canadian Museum of Nature, Ottawa, ON K1P 6P4, Canada \\ ${ }^{2}$ Department of Biology, York University, 4700 Keele St., Toronto, ON M3J 1P3, Canada \\ ${ }^{3}$ Florida State Collection of Arthropods, 1911 SW 34 ${ }^{\text {th }}$ St., Gainesville, FL 32608-1268, USA \\ *Corresponding author: thomas.onuferko@gmail.com \\ ${ }^{2}$ Email: xeromelissa@gmail.com \\ 33Email:polimita@hotmail.com \\ ${ }^{1}$ urn:lsid:zoobank.org:author:6E4CC25A-AD82-42D3-9846-C659EDEAF541
${ }^{2}$ urn:lsid:zoobank.org:author:06E03CC1-20DE-4968-8637-772B09832079
${ }^{3}$ urn:lsid:zoobank.org:author:A8ED1AB5-A208-4CD3-BE2C-0EAD8B19AA2B
}

\begin{abstract}
The bee genus Brachymelecta Linsley, 1939 has until now been represented by a single specimen, which has puzzled melittologists since its original description as Melecta? mucida Cresson, 1879. Through detailed morphological comparison and images, we show that the holotype is no more than an unusual specimen of a widespread species, most recently known as Xeromelecta californica (Cresson, 1878). We demonstrate that the diagnostic generic features for Brachymelecta fall within the range of morphological variation observed within X. californica. Although the names Brachymelecta and Xeromelecta Linsley, 1939 were published simultaneously, the former was proposed as a genus whereas the latter was proposed as a subgenus of Melecta Latreille, 1802. Thus, the following synonymies are proposed: Xeromelecta syn. nov. under Brachymelecta (since precedence must be given to the name that originally had the higher taxonomic rank) and M.? mucida syn. nov. under B. californica. Additionally, we present updated taxon concepts for and an identification key to the six known species now in Brachymelecta-B. alayoi (Michener, 1988), B. californica, B. haitensis (Michener, 1948), B. interrupta (Cresson, 1872), B. larreae (Cockerell, 1900), and B. tibialis (Fabricius, 1793) - along with redescriptions and a diagnosis for the genus. The male of $B$. alayoi and females of $B$. haitensis and B. tibialis are described for the first time. Furthermore, a phylogeny based on combined molecular and morphological data is proposed for Brachymelecta, and the evolution of the genus is explored in a historical biogeographic context.
\end{abstract}

Keywords. Brachymelecta mucida, DNA barcode, identification key, integrative biosystematics, Melectini. 


\section{Introduction}

For the past 70 years, the cleptoparasitic tribe Melectini (Hymenoptera: Apidae) has been represented in North America by four genera: Brachymelecta Linsley, 1939, Melecta Latreille, 1802, Xeromelecta Linsley, 1939, and Zacosmia Ashmead, 1898 - of which three (Melecta excepted) are known exclusively from the New World (Hurd \& Linsley 1951). The tribe was until very recently placed in the subfamily Apinae, which was rendered paraphyletic in the first molecular phylogeny of Apidae (Cardinal et al. 2010) and subsequently moved to the subfamily Nomadinae by Bossert et al. (2019). Brachymelecta and Zacosmia have each been represented by a single species, B. mucida (Cresson, 1879) and Z. maculata (Cresson, 1879), respectively. Whereas the latter is widespread and generally wellrepresented in collections, the former is known from just a single specimen, collected on an unknown date by "Morrison" (according to the original description) and with "Nev." the only information given on the collection label, understood to mean Nevada, the locality given in the original description. H.K. Morrison is known to have collected insects in Nevada in 1878 (Mann 1885), the year before B. mucida was described (as Melecta? mucida).

Features exhibited by the M.? mucida holotype that are unusual among North American Melectini include the presence of only two submarginal cells on each of the two fore wings and pale tomentum that obscures almost the entire dorsal surface of the metasoma (Michener 2007). In the most recent taxonomic assessment of B. mucida, Engel \& Michener (2012) concluded Brachymelecta to be a valid genus, separate from Melecta, emphasizing the presence of two (as opposed to three) submarginal cells on each fore wing. In all other melectine genera except Sinomelecta Baker, 1997, the fore wings typically each have three submarginal cells. Additionally, at least the discs of the metasomal terga are to some extent covered in brown or black hairs. Sinomelecta consists of a single species, S. oreina Baker, 1997 (known from only two specimens from the Hengduan Mountains in China), which is very dissimilar to $B$. mucida despite having the same number of submarginal cells. Notable differences include 11 flagellomeres in male B. mucida as opposed to 10, the shorter length of the first flagellomere relative to the second in B. mucida (about 1.6 times the length of the second as opposed to twice the length in S. oreina), the absence of well-defined apical/subapical bands of suberect pale hairs on the metasomal terga in B. mucida (present and distinct on most metasomal terga in $S$. oreina), the presence of small lateral spines on the apodemes of the male seventh sternum in B. mucida, and the slightly emarginate (as opposed to deeply notched) apices of the male seventh and eighth sterna in B. mucida (Engel \& Michener 2012).

A recent visit by the primary author of the present study to the Academy of Natural Sciences of Drexel University Entomology Collections in Philadelphia, PA, USA resulted in the discovery of a series of Xeromelecta californica (Cresson, 1878) in the main collection exhibiting variation in the length of the second submarginal crossvein on the fore wing, not only among specimens but in some cases between the left and right sides of the same specimen. Hence, the series includes specimens in which both fore wings have three submarginal cells, both fore wings have two submarginal cells, and one fore wing has three whereas the other has two submarginal cells. Moreover, this variation was observed among specimens that bear the same collection label stating only "Nev." (Fig. 1A), identical to that associated with the holotype of M.? mucida (Fig. 1B), which is housed in the same institution.

Linsley (1939) proposed the names Brachymelecta and Xeromelecta, each for a single species, although whereas Brachymelecta was presented as a new genus, Xeromelecta, into which only M. larreae (Cockerell, 1900) was placed, was presented as a subgenus of Melecta. Subsequently, Hurd \& Linsley (1951) raised Xeromelecta from subgenus to genus and, for unclear reasons, gave the name precedence over Melectomorpha Linsley, 1939, which was also originally described as a subgenus of Melecta (and published simultaneously with Xeromelecta). Melectomorpha originally included four species that all came to be regarded as Xeromelecta (subgenus Melectomorpha): M. californica, M. interrupta Cresson, 
1872, M. pasadenensis (Cockerell, 1910) (subsequently synonymized under X. californica), and M. rociadensis Cockerell, 1904 (subsequently synonymized under X. interrupta). Xeromelecta larreae became the only representative of Xeromelecta (Xeromelecta), whereas the only melectines known from the Caribbean, which are also the only exclusively Neotropical species of Melectini, were placed in the subgenus Nesomelecta (Michener, 1948). Thus, the genus Xeromelecta came to be represented by the following species (as of Ascher \& Pickering 2020): X. (M.) californica, X. (M.) interrupta, and X. (X.) larreae from North America and X. (N.) alayoi Michener, 1988, X. (N.) haitensis (Michener, 1948), and X. (N.) tibialis (Fabricius, 1793) from the Greater Antilles.

Before the names Brachymelecta and Xeromelecta were proposed, Fox (1893), in reference to the holotype of M.? mucida, remarked that "In the synoptical table here given of our species of Melecta, I have included $M$. (?) mucida, which, although possessing but two submarginal cells, is referable to Melecta. It is probably abnormal, as sometimes Melecta californica has but two submarginal cells in one wing, while in the other wing there is the usual number".

In light of this information, we set out the following objectives: a) to determine whether $B$. mucida is a valid species, and thereby whether Brachymelecta is a valid genus, b) to resolve the taxonomic understanding of all species that were at one point placed in Xeromelecta, $\mathrm{c}$ ) to provide up-to-date taxon concepts (sensu Meier 2017, see Packer et al. 2018) for them, and d) to reconstruct their evolutionary history through phylogenetic and biogeographic analysis.

\section{Material and methods}

Species limits were established using an integrative biosystematics approach, in which both morphological and molecular evidence were considered (e.g., as in Gibbs 2009, 2010, 2011; Rocha-Filho \& Packer 2015; Monckton 2016; Onuferko 2017, 2018, 2019; Ferrari 2017, 2019). Specifically, external morphology was compared among representatives of the studied species/forms of Melectini (under a Leica M80 stereo microscope). Sequences of a region of the cytochrome c oxidase subunit I (COI) mitochondrial gene (DNA barcode, Hebert et al. 2003a, 2003b) were also obtained for most species. Most DNA barcoding was carried out at the Centre for Biodiversity Genomics (CBG) at the University of Guelph in Guelph, Ontario, Canada. For barcoding, a single leg was removed (usually the midleg) from the right side of a particular (pinned) voucher specimen, the legs were stored in wells containing $\geq 95 \%$
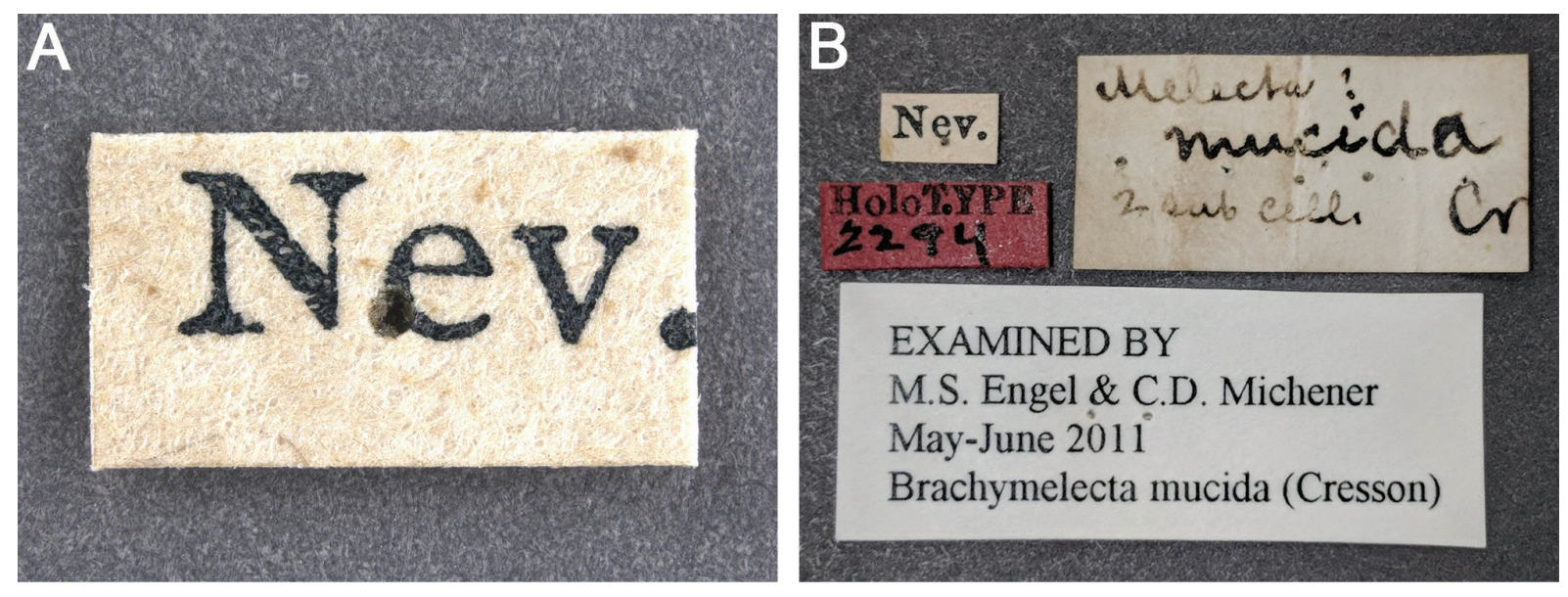

Fig. 1. Specimen labels. A. Label associated with a male specimen (Fig. 8A) in which both fore wings have two submarginal cells, found among the collection of 'Xeromelecta californica' specimens at the ANSP. B. Labels associated with the Melecta? mucida holotype, $\widehat{\partial}$ (ANSP 2294) (herein synonymized under Brachymelecta californica (Cresson, 1878)). 
ethanol, and plates were sent to the CBG for DNA extraction, PCR, sequencing, and sequence assembly and cleanup. High-quality sequences were, in an automated process, assigned barcode index numbers (BINs) representing molecular operational taxonomic units (MOTUs), which usually correspond with real species (Ratnasingham \& Hebert 2007, 2013). DNA barcoding was also carried out in part by the primary author at the Canadian Museum of Nature (CMN) Laboratory of Molecular Biodiversity, which involved a different procedure described as follows. A single pinned, dried specimen was soaked in $\geq 95 \%$ ethanol in a freezer (between $-10^{\circ} \mathrm{C}$ and $-20^{\circ} \mathrm{C}$ ) for several days, after which the pin was removed, the head, prosternum, propleura, and fore legs were detached (and later reattached with Elmer's Washable Clear School Glue for vouchering purposes), and the softened muscle tissues were extracted with flamesterilized fine-tipped forceps. DNA was then extracted from the tissues following a procedure for animal DNA extraction adapted from Ivanova et al. (2006). To amplify the DNA barcode region, the primer pairs LepF1 / LepR1 (Hebert et al. 2004) (to amplify the full sequence) and MLepF1 (Hajibabaei et al. $2005)$ / LepR1 (to amplify a shorter fragment) were used. The reaction mixture (15 $\mu \mathrm{L}$ in total) consisted of 7.05-9.05 $\mu \mathrm{L}$ (depending on the amount of DNA template used) DNA-grade water, $3 \mu \mathrm{L} 5 \times \mathrm{Q}^{\circledR}$ Reaction Buffer (New England Biolabs (NEB), Ipswich, Massachusetts, USA), $0.3 \mu \mathrm{L}$ dNTPs (10 mM), $0.75 \mu \mathrm{L}$ forward primer $(10 \mu \mathrm{M}), 0.75 \mu \mathrm{L}$ reverse primer $(10 \mu \mathrm{M}), 0.15 \mu \mathrm{L}$ Q5 ${ }^{\circledR}$ High Fidelity DNA polymerase (0.3 U/rxn) (NEB), and 1-3 $\mu \mathrm{L}$ DNA template. Amplification of the DNA barcode region used the following PCR conditions programmed into an Eppendorf ${ }^{\circledR}$ Mastercycler ${ }^{\circledR}$ Pro Thermal Cycler: an initial denaturation at $94^{\circ} \mathrm{C}$ for 1 minute, five cycles of denaturation at $94^{\circ} \mathrm{C}$ for 40 seconds, annealing at $45^{\circ} \mathrm{C}$ for 40 seconds, and extension at $72^{\circ} \mathrm{C}$ for 1 minute followed by 35 cycles of denaturation at $94^{\circ} \mathrm{C}$ for 40 seconds, annealing at $51^{\circ} \mathrm{C}$ for 40 seconds, and extension at $72^{\circ} \mathrm{C}$ for 1 minute, and a final extension at $72^{\circ} \mathrm{C}$ for 5 minutes. Sequencing reactions for Sanger sequencing of amplicons in both directions (using the same primers used in PCR), purification of sequencing products, and automated $\mathrm{ABI}$ sequencer runs were carried out at the CMN using the protocol described in Onuferko et al. (2019). The resulting traces were imported into Geneious ver. 11.1.5 (Kearse et al. 2012) for assembly into a consensus sequence, which was checked therein for quality, contamination, and to ensure that in the correct reading frame no stop codons were present. Sequence similarity/dissimilarity was visualized in a neighbor-joining tree based on the Tamura-Nei genetic distance model (Tamura \& Nei 1993), which was also generated in Geneious. Sequences are published in the 'New World Melectini' project on the Barcode of Life Data System (BOLD) (https://www.boldsystems.org/) and have also been deposited in GenBank (https://www.ncbi.nlm.nih.gov/genbank/) (for the complete list of sequenced specimens and available corresponding accession numbers, see Supp. file 1.).

Terminology follows Michener (2007) except, following Prentice (1998) and other recent papers (e.g., Mir Sharifi et al. 2019), the frons, gena, and vertex are herein referred to as the frontal, genal, and vertexal areas, respectively, since these features are not clearly demarcated on the surface of the head. Additional terms used in this study, some of which are taken from previous taxonomic studies of cleptoparasitic bees (Rightmyer 2008; Onuferko 2017, 2018, 2019) are redefined here as follows: in the species descriptions, length refers to measurements made along the longitudinal axis of a bee and width refers to measurements made along the lateral axis, except that breadth is used rather than length in reference to the longitudinal extent of the bands of short, appressed branched pale hairs (fasciae) present on the metasomal terga of most of the treated species. Genal beard refers to the long, erect hairs occupying much of the genal area. In two of the treated species, the mesoscutum has a pair of anterior longitudinal bands of hairs darker than the surrounding off-white or yellow-orange hairs, herein referred to as paramedian bands. Bigibbous describes the mesoscutellum of Brachymelecta and Xeromelecta, which has a pair of convexities (in most species, each of these narrows into a prominent spine or mammiform tubercle, which is oriented posteriorly). 


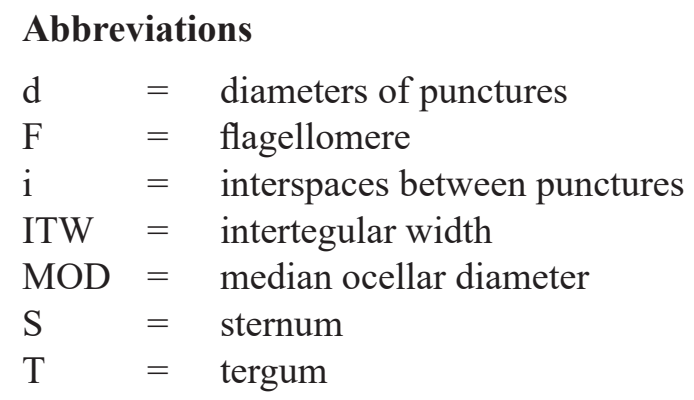

A calibrated eyepiece graticule was used to measure various anatomical features, always at their longest and/or widest extents. The scape was measured without the radicle.

The primary types of all the valid species treated herein and their junior synonyms were examined personally by the primary author except the holotypes of Crocisa pantalon Dewitz, 1881 and Xeromelecta alayoi, which were studied from detailed images provided by the collections staff at the institutions where the specimens are housed (see Acknowledgements). The redescription of each species is based on the primary type specimen (holotype or lectotype); however, since the holotype of $X$. alayoi was unavailable for detailed study under the microscope, measurements of anatomical features presented for that species are based on a non-type female specimen (the only one available for study). Redescriptions follow the format of Onuferko $(2017,2018,2019)$, with a full description provided for the sex that corresponds to the primary type specimen and a shorter description provided for the sex opposite that of the primary type that lists only key differences. Full descriptions for both sexes are not provided since the studied species do not exhibit marked sexual dimorphism except in the terminalia and usual secondary sexual characters (number of flagellomeres; presence of deep, pit-like flagellar sensoria in males but not females; and number of metasomal segments). It should be noted that in males of the species that were placed in Xeromelecta the pedicel is often partially if not entirely recessed into the scape, such that the scape appears to join F1 directly. Hence, in sexing specimens one must be careful not to mistake F1 for the pedicel, which is very short (much wider than long), whereas F1 is as long as or longer than it is wide.

A morphological diagnosis is provided for the nominal genus to distinguish it from all other bees in North America and the Caribbean. In addition to the diagnoses provided for each of the treated species, females and males can be identified to species with the fully illustrated dichotomous key presented herein. Color images were taken using the Leica Z16 APO A imaging system; focus stacked using the accompanying LAS software; minimally edited for brightness/contrast, resized, and cropped in PaintShop Pro (Jasc Software, Inc.); and assembled into figure plates and labelled in Adobe Photoshop 2020 (Adobe Inc.). For scanning electron microscopy (SEM), pinned specimens were mounted onto SEM stubs. The pins were inserted at an angle through a small piece of Styrofoam attached to a round aluminum stub with foil tape. The specimens' mouthparts were examined and imaged uncoated with an Apreo FEI field emission microscope (FEI, Hillsboro, USA). The study was conducted using voltages between 3 and $6 \mathrm{kV}$ and a working distance of $\sim 30 \mathrm{~mm}$. Secondary electron (SE) imaging was selected over backscatter (T1), giving a clearer 3D perspective of the maxillary palpus.

Specimens were studied from 15 entomological collections (in Europe and North America).

\section{Abbreviations for institutions/collections housing studied specimens}

Names of contacts (curators and/or collections managers) are given in parentheses.

$\begin{aligned} \text { ANSP }= & \text { Academy of Natural Sciences of Drexel University, Philadelphia, PA, USA (J.D. } \\ & \text { Weintraub) } \\ \text { CAS } \quad= & \text { California Academy of Sciences, San Francisco, CA, USA (C. Grinter and R. Zuparko) }\end{aligned}$




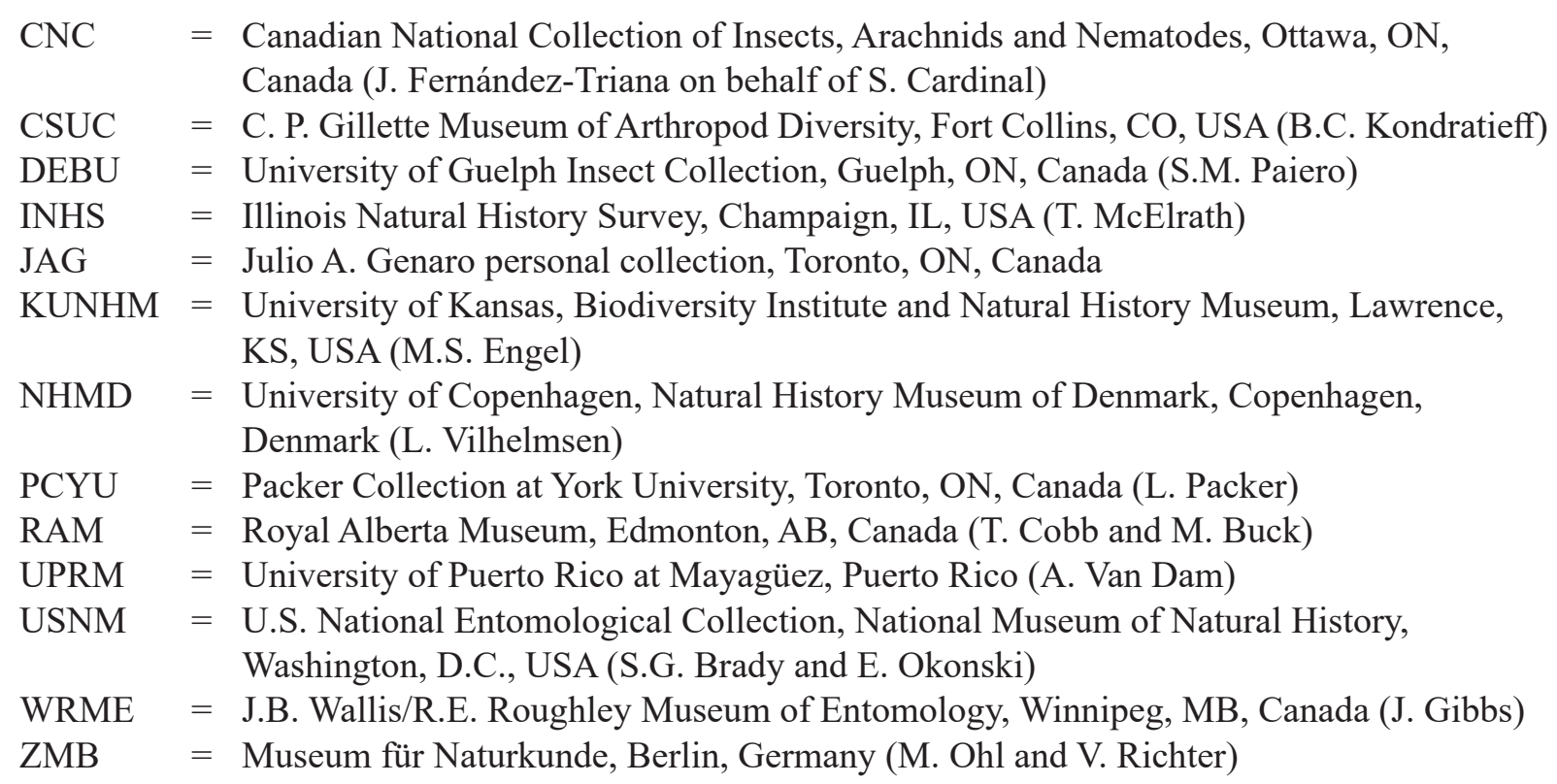

RStudio (ver. 1.1.463) was used to generate species distribution maps from georeferenced collection and observational records using the packages maptools (Bivand \& Lewin-Koh 2018), raster (Hijmans 2018), and rgeos (Bivand \& Rundel 2018), which were installed in R (ver. 3.5.2) (R Core Team 2018). Shapefiles for the projected political and geographic boundaries were obtained from DIVA-GIS (http://www.diva-gis.org/gdata), GADM data (ver. 3.6) (https://gadm.org/data.html), Statistics Canada (2015), and the US Census Bureau (2015). GPS coordinates were obtained directly from collection labels or approximated from the named locality if missing from a collection label or if missing from a named locality associated with an image. In some cases, voucher specimens only contained vague information about the collection locality (e.g., only an abbreviation for the state in which a specimen was collected). Hence, in addition to presenting individual georeferenced occurrence records on each map (using colored circles), for species occurring on the mainland the entire subnational entity (province or state) in which the species in question has been collected or observed has been colored in.

To construct a phylogeny for the genus, all species previously placed in Xeromelecta and four outgroup taxa (all of which are Melectini, including Melecta albifrons (Forster, 1771), M. thoracica Cresson, 1875, Thyreus novaehollandiae (Lepeletier, 1841), and Zacosmia maculata) were scored for 28 morphological characters (Supp. file 2). This dataset (Table 1) was combined with a matrix of available DNA barcode sequences aligned in Geneious using the Geneious (multiple) Alignment option. Full-length sequences for all outgroup taxa were available and included (BOLD sample IDs: BMNH(E)\#970451, LRBBC986, 03-UT-2432, CCDB-14506-A6). DNA barcodes for T. novaehollandiae are newly published whereas those for the other outgroups were downloaded from public records on BOLD. Sequences for the ingroup taxa (BOLD sample IDs: 07-ID-1195, CMNTO_037, CCDB-34570 B04, CCDB-29918 H07) are newly published herein and available for all but two species (both previously placed in the subgenus Nesomelecta). Except for one partial sequence 338 base pairs (bp) in length (the only one available for the subgenus Xeromelecta), all sequences included in the molecular matrix are $658 \mathrm{bp}$ in length. The sampled melectines were identified to genus using Michener (2007) (and species in the case of Zacosmia since the genus is monotypic). Species of Melecta from the Nearctic and Palearctic were identified using the keys and with reference to the descriptions of Hurd \& Linsley (1951) and Lieftinck (1980), respectively. Specimens of T. novaehollandiae were identified as such with reference to the taxon concept for the species provided in Lieftinck (1962). Information about the voucher specimens is provided in Supp. file 1. In the phylogenetic reconstruction, the root was set to Zacosmia maculata based on its placement as sister to the node that includes Melecta, Thyreus Panzer, 1806, and Xeromelecta in 
Table 1. Matrix of morphological characters used in phylogenetic analysis in TNT. Numbers in column headers indicate character identifiers (see Supp. file 2 for the corresponding descriptions). Character states separated by a comma indicate polymorphisms. Unknown character states and states that were not scored for certain taxa because they are inapplicable are denoted by question marks.

\begin{tabular}{|c|c|c|c|c|c|c|c|c|c|c|c|c|c|c|c|c|c|c|c|c|c|c|c|c|c|c|c|c|}
\hline & & & & & & & & & & & 1 & 1 & 1 & 1 & 1 & 1 & 1 & 1 & 1 & 1 & 2 & 2 & 2 & 2 & 2 & 2 & 2 & 2 \\
\hline & 0 & 1 & 2 & 3 & 4 & 5 & 6 & 7 & 8 & 9 & 0 & 1 & 2 & 3 & 4 & 5 & 6 & 7 & 8 & 9 & 0 & 1 & 2 & 3 & 4 & 5 & 6 & 7 \\
\hline Z. maculata & 0 & 0 & 0 & 0 & 0,1 & 0 & 0 & $?$ & 0 & 0 & 0 & 0 & 0 & $?$ & 0 & 0 & 0 & 0 & 0 & 0 & 0 & 0 & 0 & 0 & 0 & 0 & 0 & 0 \\
\hline T. novaehollandiae & 1 & 0 & 0 & 1 & 0 & 2 & 1 & 0 & 0 & 1 & 1 & 1 & 0 & $?$ & 1 & 0 & 0 & 1 & 1 & 0 & 0 & 0 & 0 & 0 & 0 & 0 & 1 & 1 \\
\hline M. albifrons & 0 & 1 & 1 & 1 & 2 & 1 & 1 & 1 & 0 & 0 & 1 & 1 & 1 & 0 & 1 & 1 & 1 & 0 & 1 & 1 & 1 & 1 & 1 & 1 & ? & 0 & 1 & 1 \\
\hline M. thoracica & 0 & 1 & 1 & 1 & 2 & 1 & 1 & 1 & 0 & 0 & 0 & 1 & 1 & 0 & 1 & 1 & 1 & 0 & 1 & 0 & 0 & 1 & 1 & 1 & $?$ & 0 & 0 & 1 \\
\hline B. alayoi & 1 & 0 & 1 & 0 & 0 & 2 & 1 & 0 & 0 & 1 & 1 & 1 & 1 & 0 & 1 & 0 & 1 & 0 & 0 & 0 & 1 & 1 & 0 & 0 & 0 & 0 & 1 & 0 \\
\hline B. californica & 1 & 1 & 0 & 0 & 1 & 2 & 0 & 0 & 0 & 0 & 1 & 1 & 1 & 1 & 1 & 0 & 1 & 0 & 1 & 1 & 1 & 1 & 0 & 0 & 1 & 1 & 1 & 0 \\
\hline B. haitensis & 1 & 0 & 1 & 0 & 0 & 2 & 1 & 0 & 1 & 1 & 1 & 2 & 1 & 0 & 2 & 0 & 1 & 0 & 0 & 1 & 1 & 1 & 0 & 0 & 0 & 0 & 1 & 0 \\
\hline B. interrupta & 1 & 1 & 0 & 1 & 1 & 2 & 0 & 0 & 0 & 0 & 1 & 1 & 1 & 1 & 1 & 0 & 0 & 1 & 1 & 1 & 1 & 1 & 0 & 0 & 1 & 1 & 1 & 0 \\
\hline B. larreae & 0 & 1 & 0 & 1 & 2 & 1 & 0 & 0 & 0 & 0 & 0 & 1 & 0,1 & 1 & 1 & 0 & 0 & 1 & 1 & 0 & 1 & 1 & 0 & 0 & 0 & 1 & 0 & 0 \\
\hline B. tibialis & 1 & 0 & 1 & 0 & 0 & 2 & 1 & 0 & 1 & 1 & 1 & 2 & 1 & 0 & 2 & 0 & 1 & 0 & 0 & 1 & 1 & 1 & 0 & 0 & 0 & 0 & 0 & 0 \\
\hline
\end{tabular}

the comprehensive molecular phylogeny of Apidae published by Cardinal et al. (2010). Phylogenetic analysis was carried out in TNT ver. 1.1 (Goloboff et al. 2008) (made available by the Willi Hennig Society), initially using the implicit enumeration algorithm, with characters weighted equally and character states non-additive, then using extended implied weighting (Goloboff 2014), which allows for improved treatment of datasets that include both molecular and morphological partitions. For the latter, weights were selected using the script 'setk.run', written by J. Salvador Arias (Instituto Miguel Lillo, San Miguel de Tucumán, Argentina) and used and first referenced in Santos et al. (2015), with k estimated separately for the molecular and morphological data partitions. With space set aside for 10000 trees in memory, a new technology search for the most parsimonious tree(s), using the parsimony ratchet (Nixon 1999), tree drifting (Goloboff 1999), and tree fusion (Goloboff 1999), was conducted, with hits to minimum length set to 1000 and the random seed set to 0. Absolute Bremer support (Bremer 1994) was calculated in the same program using the accompanying macro 'BREMER.RUN'. Additionally, branch support as GC values (frequency differences) was determined by symmetric resampling (Goloboff et al. 2005 ) with 10000 replicates. The single most-parsimonious tree or consensus tree was visualized and edited in WinClada 1.00.08 (Nixon 2002).

To study the evolution of the target genus in a historical biogeographic context, spatial analysis of vicariance, implemented in the Vicariance Inference Program (VIP) (Arias et al. 2011), was used to identify disjunct sister nodes in the phylogenetic tree. Thereby, instances of allopatric speciation (whether by dispersal or vicariance) were inferred. The tree file produced in TNT was converted to an XML file in the same program using the macro 'toxml.run' (available at http://www.lillo.org.ar/phylogeny/VIP/downs.htm) and opened in VIP. Subsequently, a list of (actual and approximate) geographic coordinates signifying known reliable locality records for the six species comprising the ingroup (see Supp. file 1.) were fed into VIP. A grid size of $0.5^{\circ} \times 0.5^{\circ}$ was specified for the analysis, which given the dataset corresponds to a north-south distance of $55.4 \mathrm{~km}$ and an east-west distance of $32.5-52.8 \mathrm{~km}$ (varying because the distance between lines shortens toward the poles). A maximum fill of 1 (the default) was selected, expanding the expected area of occurrence to include the eight cells surrounding the cell wherein the actual record falls (Moore neighborhood). As in Monckton (2016) and Onuferko et al. (2019), the maximum amount of range overlap allowed between sister taxa 
was increased from $0 \%$ (the default value) to $25 \%$ to allow for the possibility that a taxon partially extended its range into the area occupied by its sister taxon due to the barrier to gene flow having been interrupted sometime after the sister taxa speciated in allopatry. With default settings used for the remaining parameters, a heuristic search was carried out with 1000 iterations.

\section{Results}

\section{Taxonomy}

Class Insecta Linnaeus, 1758

Order Hymenoptera Linnaeus, 1758

Superfamily Apoidea Latreille, 1802

Family Apidae Latreille, 1802

Subfamily Nomadinae Latreille, 1802

Tribe Melectini Westwood, 1840

Genus Brachymelecta Linsley, 1939

Brachymelecta Linsley, 1939: 458.

Xeromelecta Linsley, 1939: 450, syn. nov.

Melectomorpha Linsley, 1939: 451, syn. nov.

Nesomelecta Michener, 1948: 15, syn. nov.

\section{Diagnosis for Brachymelecta}

Brachymelecta (previously Xeromelecta) is an exclusively New World genus of small to mid-sized $(8-16 \mathrm{~mm})$ non-metallic anthophoriform bees, for which the following morphological features in combination constitute a diagnosis: the inner ramus of each tarsal claw of the mid- and hind legs is broad, lobe-like, and thus does not resemble the outer ramus (Fig. 2A-B) (this feature is unique among North American Melectini but also exhibited by many species of Melecta from the Old World (e.g., M. albifrons) as well as many other groups of cleptoparasitic bees, Michener 2007); the distitarsi of all legs have arolia (Hurd \& Linsley 1951: pl. 11f); the marginal cell of each fore wing extends little (if at all) beyond the third submarginal crossvein (or, if the wing has only two submarginal cells, the second submarginal crossvein, which is equivalent morphologically to the third because it is the second that is lost) (e.g., Fig. 3A; see also Engel \& Michener 2012: fig. 4 to see this feature in the M.? mucida holotype); vein cu-v of each hind wing is distinctly longer than the second abscissa of vein $\mathrm{M}+\mathrm{Cu}$ (Engel \& Michener 2012: fig. 5) (this feature is unique to Ericrocidini, Melectini, and Rhathymini, RoigAlsina \& Michener 1993; Michener 2007); in both pairs of wings, most closed cells are hairless and the region beyond the veins of each wing is strongly papillate (e.g., Fig. 3A; see also Engel \& Michener 2012: figs 4-5 to see this feature in the M.? mucida holotype); and most pale hairs (if present) on T1 are distinctly shorter than the longest hairs on the mesosoma (e.g., Fig. 3A-C).

All species of Brachymelecta except B. larreae have well-defined metasomal fasciae comprised of short, appressed, branched pale hairs (e.g., Fig. 3A-C), and may thus superficially resemble other cleptoparasitic Apidae (subfamily Nomadinae), most notably various Epeolini, Ericrocis Cresson, 1887 (Ericrocidini), and Hexepeolus Linsley \& Michener, 1937 (Hexepeolini). Brachymelecta can be readily distinguished from the aforementioned taxa by the length of the marginal cell of the fore wing, which in the non-melectine nomadines extends well beyond the third submarginal crossvein (or second submarginal crossvein if the wing has only two submarginal cells). In contrast to Epeolini and Hexepeolus (but not Ericrocis), female Brachymelecta in ventral view show six recognizable metasomal sterna, with S6 tapering (in female Nomadinae sensu Michener 2007, S6 is largely if not entirely retracted 
and commonly emarginate or bifid). Brachymelecta can be further distinguished from any Epeolini by their axillae, which in the latter are produced to rounded lobes, angles, or spines (in Brachymelecta, the axillae continue the contour of the mesoscutellum, as in Ericrocis and Hexepeolus). Species of Brachymelecta, especially B. larreae, may also be confused with Melecta, but in New World Melecta spp. the inner ramus of each tarsal claw is narrow, pointed, and thus resembles the outer ramus (Fig. 2C), and T1 has long, pale hairs, similar in length to those on the dorsum of the mesosoma. Additionally, whereas in male Brachymelecta from North America each flagellomere except F1 is distinctly wider than long $(\mathrm{L} / \mathrm{W}$ ratio $\leq 0.8)$, in male Melecta each flagellomere is at most as wide as long $(\mathrm{L} / \mathrm{W}$ ratio $=$ 0.9-1.0). In males of the Antillean species of Brachymelecta, the flagellomeres are longer, as in Melecta, but given that the three species are the only melectines known to occur in the Caribbean, they can easily be separated from all other melectine genera by geography. The absence of arolia in Zacosmia readily distinguishes the genus from both Brachymelecta and Melecta.

\section{Remarks}

We present Brachymelecta as the replacement name for Xeromelecta at the generic level. This nomenclatural act was prompted by the recognition of the lectotype of Melecta californica and holotype of M.? mucida as belonging to the same species. The holotype of M.? mucida agrees with the diagnosis for the genus Brachymelecta provided herein, which corresponds to the former genus Xeromelecta. Most notably, the inner ramus of each tarsal claw of the mid- and hind legs is broad, lobe-like, and thus does not resemble the outer ramus, as in all species formerly placed in Xeromelecta (see Fig. 2A-B). In their redescription of Brachymelecta mucida, Engel \& Michener (2012) also noted the similarity between the tarsal claws of the M.? mucida holotype and X. californica.

Evidence that the morphological features exhibited by the holotype of M.? mucida fall within the range of variation observed within B. californica is presented in the 'Remarks' section for B. californica (vide infra). Melecta californica was described earlier, so the epithet 'californica' is the senior synonym to be used in reference to the species. Prior to the present study, the name used for this species was Xeromelecta californica. The name Xeromelecta was elevated to the rank of a genus by Hurd \& Linsley (1951), after Brachymelecta had already been established as a generic name, by Linsley (1939). The two names were
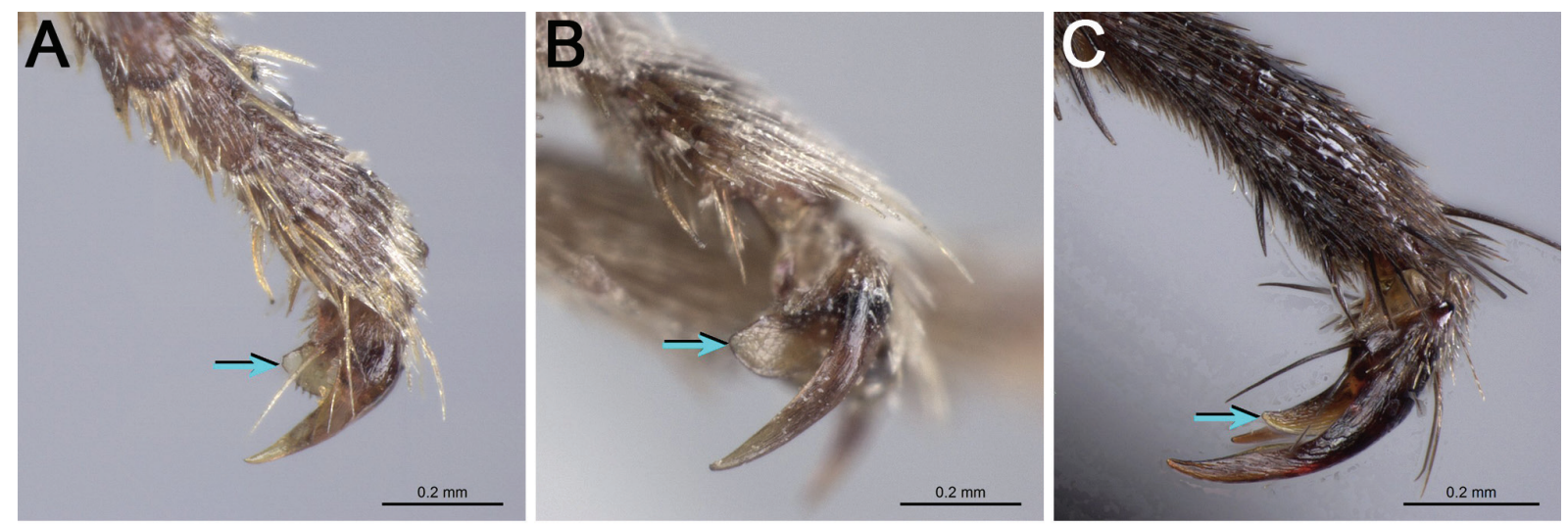

Fig. 2. Metadistitarsus of male, lateral view. A. Brachymelecta californica (Cresson, 1878) (photo of Melecta? mucida holotype (ANSP 2294), herein synonymized under B. californica), in which the inner ramus (blue arrow) of each tarsal claw is broad, lobe-like, and thus does not resemble the outer ramus. B. B. californica (photo of M. californica lectotype (ANSP 2292)), in which the inner ramus (blue arrow) of each tarsal claw is broad, lobe-like, and thus does not resemble the outer ramus. C. Melecta thoracica Cresson, 1875 (BOLD sample ID: LRBBC1476; PCYU LRB09-3186), in which the inner ramus (blue arrow) of each tarsal claw is narrow, pointed, and thus resembles the outer ramus. 
published simultaneously, but Xeromelecta was originally described as a subgenus of Melecta whereas Brachymelecta was described as a separate genus altogether into which M.? mucida was placed, the first among what are now understood to be its congeners to be placed in a new genus. According to article 24.1. of the code of the International Commission on Zoological Nomenclature (ICZN), when synonyms are published simultaneously but proposed at different ranks, the name proposed at the higher rank takes precedence. Therefore, even though M.? mucida is herein regarded as a junior synonym at the species level, Brachymelecta is regarded as the senior synonym for the genus.

Since B. mucida was established as the type species of its genus by original designation and monotypy, its senior synonym (B. californica) is herein regarded as the type species for the genus Brachymelecta. Under its former genus, the type species was placed in the subgenus Melectomorpha. Since article 44 of the code of the ICZN stipulates that (in a genus containing subgenera) the subgenus that contains the type species must be denoted by the same name as the genus, the subgenus Melectomorpha is herein recognized as no longer valid. Besides, according to the results of our phylogenetic analysis (presented below), Melectomorpha is paraphyletic, with its two members, $B$. californica and $B$. interrupta, not sister to one another but with $B$. interrupta sister to $B$. larreae, the only species in the subgenus Xeromelecta. Although Nesomelecta was found to constitute a natural group, to maintain its status as a valid subgenus would require the other three species of Brachymelecta to each be placed in their own separate subgenus or for $B$. interrupta to be placed in the same subgenus as B. larreae. As four or even three subgenera for a genus of six species is arguably a case of overclassification, the names Xeromelecta, Melectomorpha, and Nesomelecta are herein synonymized under Brachymelecta.

Brachymelecta alayoi (Michener, 1988)

Figs 3, 4A, 17

Xeromelecta alayoi Michener, 1988: 377 (ㅇ), figs 1-4.

\section{Proposed common name}

Alayo's digger-cuckoo bee.

\section{Diagnosis}

Unique within the genus to $B$. alayoi are each of the following morphological features: the mesoscutum has a well-defined band of yellow hairs along the midline that does not attain its posterior margin (Fig. 3B, D); each mesotibia of the female has a patch of yellow hairs that occupies nearly its entire dorsal surface (Fig. 3A); and the mesoscutellum, except for the pair of spines and around their bases, is densely covered in yellow pubescence, such that the underlying integument is greatly obscured (Fig. 3D). Brachymelecta alayoi most closely resembles $B$. haitensis, a Hispaniolan species, but in $B$. haitensis the mesoscutum and mesoscutellum both have well-defined bands of pale yellow hairs along the entire midline that connect and thus give the appearance of a single band; the mesoscutellum sometimes also has pale yellow hairs laterally, but the pubescence is otherwise dark brown or black, sparse, and does not obscure the underlying integument; each mesotibia of the female dorsally has a large glabrous area between a submedial band of off-white to pale yellow hairs (nearer the base than apex) and a band on the apical margin; and the T1-T3 fasciae of the male are complete. Brachymelecta alayoi is the only species in the genus known to occur in Cuba, where it is endemic.

\section{Material examined}

Primary type material

CUBA $\bullet+$, holotype (studied from images); Pinar del Río, Rancho Mundito, Sierra de los Órganos; 4 Jul. 1947; F. de Zayas and V.J. Ferras leg.; KUNHM 1461055. 
DNA barcoded material

Unavailable.

\section{Non-barcoded material}

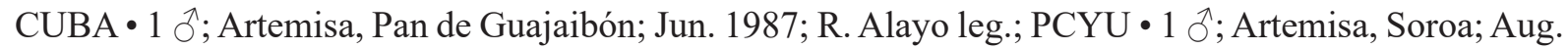
1990; J.A. Genaro leg.; USNM • 1 क; Santiago de Cuba, La Gran Piedra, Sierra Maestra; May 1955; P. Alayo leg.; JAG • 1 §; same collection data as for preceding; Jun. 2001; J.A. Genaro leg.; JAG.

\section{Redescription}

\section{Female}

Measurements. Length $11.2 \mathrm{~mm}$ (11 mm for holotype; Michener 1988); ITW $2.7 \mathrm{~mm}$; head length $2.7 \mathrm{~mm}$; head width $3.3 \mathrm{~mm}$; fore wing length $8.6 \mathrm{~mm}$ ( $9 \mathrm{~mm}$ for holotype; Michener 1988).

InTEGUMent COLORATION. Dark brown to black except as follows. Mandible with apical third golden yellow in non-type specimen. Mandible with basal two-thirds, labrum, flagellum with ventral surface (in non-type specimen), tip of mesoscutellar spine, and legs, excluding dark brown coxae and tibial spurs, reddish orange. Tegula amber. Fore wing dusky subhyaline throughout, slightly darker beyond venation. Hind wing dusky subhyaline to hyaline. Much of metasoma with reddish tinge in non-type specimen.
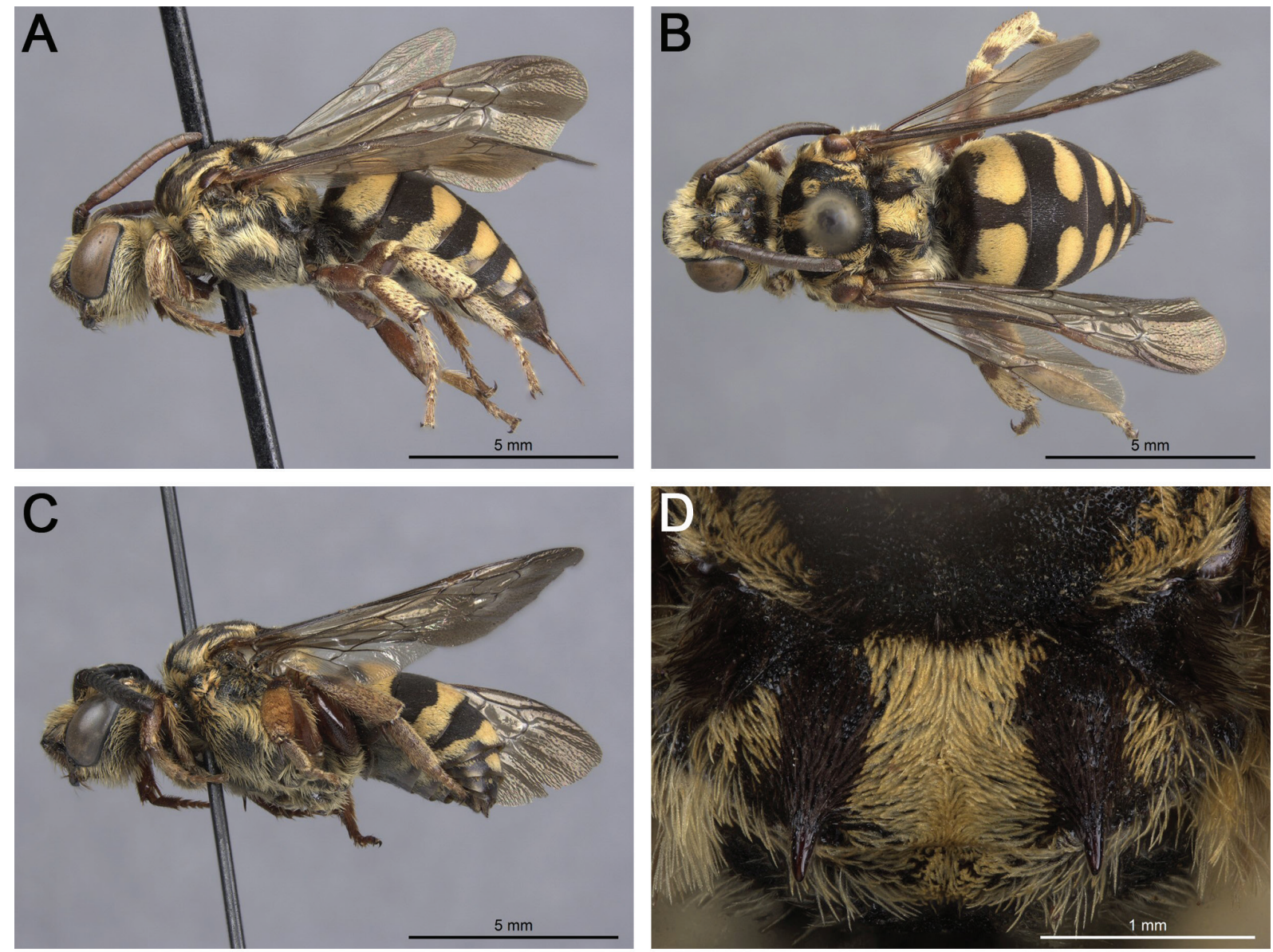

Fig. 3. Brachymelecta alayoi (Michener, 1988). A-B, D: female (JAG); C: male (JAG). A. Habitus, lateral view. B. Habitus, dorsal view. C. Habitus, lateral view. D. Axillae and mesoscutellum, dorsal view. 
PUBESCENCE. Face with hairs dense throughout except clypeus with lower quarter and area around ocelli mostly exposed, predominantly pale to golden yellow but dark brown/black around ocelli. Head with dense, erect hairs along preoccipital ridge almost entirely pale to golden yellow. Genal beard hairs predominantly pale to golden yellow. Mesoscutum sparsely hairy except densely hairy anteriorly and along margins, with hairs short, appressed, and predominantly dark brown/gray except for small patch of golden-yellow hairs on each side along anterior margin between midline and pronotal lobe, pair of central spots of golden-yellow hairs and narrow longitudinal band posterior to each extending to axilla, well-defined band of golden-yellow hairs along most of midline (not attaining posterior margin), and pale to golden-yellow hairs along margins. Axilla with conspicuous patch of black hairs. Mesoscutellum, except for pair of spines and around their bases, densely covered in golden-yellow pubescence greatly obscuring underlying integument. Metanotum with long, erect/suberect golden-yellow hairs, densest medially. Propodeum with erect, predominantly pale to golden-yellow hairs. Mesopleuron with (pale to golden-yellow) hairs moderately dense ventrally as well as between two sparsely hairy circular patches (one beneath base of fore wing (hypoepimeral area), a larger one occupying much of ventrolateral half of mesopleuron). Legs, from coxae to tarsi, with appressed and erect pale to golden-yellow hairs. Profemur with posteroventral fringe of dense, pale yellow hairs. Protibia covered in sparse to moderately dense pale to golden-yellow hairs. Mesotibia and metatibia each with patch of moderately dense short, appressed pale to golden-yellow hairs, occupying nearly entire dorsal surface (with sparsely hairy ovate patch in basal quarter). T1-T4 with well-defined medially interrupted apical fasciae, each with lobe-like anterolateral extension on each side with erect among appressed golden-yellow hairs except that of T2 with pair of anterolateral extensions on each side. T3 and T4 each with fascia laterally removed from apical margin, narrowed or interrupted mesad each anterolateral extension. T5 and T6 without fasciae. Exposed metasomal sterna mainly with short, appressed pale yellow hairs.

SURFACE SCULPTURE. Labrum and clypeus (except medially in lower quarter where sparsely punctate) with punctures equally dense (most $i \leq 1 \mathrm{~d}$ ). Clypeus with many smaller punctures among large ones. Integument lateral to lateral ocellus punctate. Mesoscutum and mesoscutellum with fine punctures, not much coarser than those of metasomal terga. Mesoscutum and mesoscutellum with punctures equally dense (most $\mathrm{i}<1 \mathrm{~d}$ ) and similar in size. Mesopleuron with denser (most $\mathrm{i}<1 \mathrm{~d})$ punctures in upper half than ventrolateral half (many $\mathrm{i}=1-2 \mathrm{~d}$ ), interspaces well-defined and shining. Discs of metasomal terga with punctures very fine, dense ( $\mathrm{i} \approx 1 \mathrm{~d}$ ), interspaces dull due to tessellate surface microsculpture.

StRUCture. Mandible tridentate, with small inner tooth approximately $1 / 3$ length of mandible from base and slightly larger inner tooth approximately $1 / 3$ length of mandible from apex as well as usual large apical tooth (rutellum) (difficult to see in holotype because mandibles closed; described from nontype specimen). Maxillary palpus apparently with one palpomere (three according to Michener 1988) (mouthparts not extended in holotype; described from non-type specimen). Scape with greatest length $2.2 \times$ greatest width. F2 nearly as long as wide $(\mathrm{L} / \mathrm{W}$ ratio $=0.9)$. Mesoscutellum strongly bigibbous, with pair of long, acute, subparallel spines, directed posteriorly. Lateral surface of propodeum posterior to spiracle with rugose crescent ridge, strongly carinate above and joining anterior lip of spiracle (difficult to see in holotype because integument obscured by dense pubescence; described from non-type specimen). Fore wing with two submarginal cells (second submarginal crossvein present but incomplete in both fore wings of all known specimens, including only paratype; Michener 1988). T6 with narrow, V-shaped but apically rounded pygidial plate with median longitudinal ridge.

\section{Male}

Description as for female except for usual secondary sexual characters and as follows: scape shorter, with greatest length $1.8 \times$ greatest width; mesotibia with patch of very dense, short, appressed, pale to golden-yellow hairs, denser than hairs on mesotibia of female and metatibia of both sexes; T5 with 
small patches of pale yellow hairs along anterior margin of apical impressed area; T7 with slight median emargination.

\section{Distribution}

This species is known only from Cuba and is the only species of Brachymelecta known to occur in the country (Fig. 4A).

\section{Ecology}

\section{Host records}

Unknown. Given that New World Melectini have been associated only with anthophorine bees (mostly Anthophora Latreille, 1803 spp.) (Hymenoptera: Apidae: Anthophorinae), presumably B. alayoi is a cleptoparasite of one or both species of Anthophora-A. atrata Cresson, 1865 and A. hilaris Smith, 1879 — known to occur in Cuba (see Brooks 1999).

\section{Floral records}

Unknown.

\section{Remarks}

The male of B. alayoi is described here for the first time. The only (female) paratype (figs 2, 4 in Michener 1988 ) is meant to have been deposited in the USNM but does not appear to be there (S.G. Brady and E. Okonski, personal communication, 2019).

Brachymelecta californica (Cresson, 1878)

Figs 1, 2A-B, 4B, 5, 6A-B, 7-8, 12A, 17

Melecta californica Cresson, 1878: 91 (, đ̊); Cresson, 1916: 114 (ð) (lectotype designation). Melecta? mucida Cresson, 1879: 205 (ठ), syn. nov.

Melecta miranda Fox, 1893: 143 (ㅇ).

Pseudomelecta pasadenensis Cockerell, 1910: 27 ( $($ ) $)$.

Melecta sladeni Viereck, 1924: 15 ().

\section{Proposed common name}

California digger-cuckoo bee.

\section{Diagnosis}

The following morphological features in combination can be used to tell B. californica apart from all other Brachymelecta: the mesoscutum has a pair of anterior spots or (paramedian) bands of hairs darker than the surrounding off-white hairs (Fig. 5B); the mesoscutellum has a pair of short, mammiform tubercles (Fig. 5D); the fore wings are infuscate apically, with most cells as well as the membrane around the third submarginal crossvein (or second submarginal crossvein if the wing has only two submarginal cells) and second recurrent vein subhyaline (Fig. 5A-C); and T3 and T4 each have a medially interrupted fascia that is narrowed or interrupted (as opposed to continuously expanded) laterally, mesad the inner pair of anterolateral extensions (there are two such lobe-like extensions on each side) (Fig. 5A-C). Brachymelecta californica is most similar to B. interrupta, but in B. interrupta the fore wings are infuscate throughout except around the third submarginal crossvein and second recurrent vein, where they are subhyaline, and $\mathrm{T} 3$ and $\mathrm{T} 4$ each have a medially interrupted fascia that is broadened anterolaterally, that of T3 into the inner pair of anterolateral extensions. Additionally, in B. interrupta the lighter hairs covering the head, mesosoma, and metasoma range from pale yellow to yellow orange, whereas in B. californica they are off-white or very rarely pale yellow. 

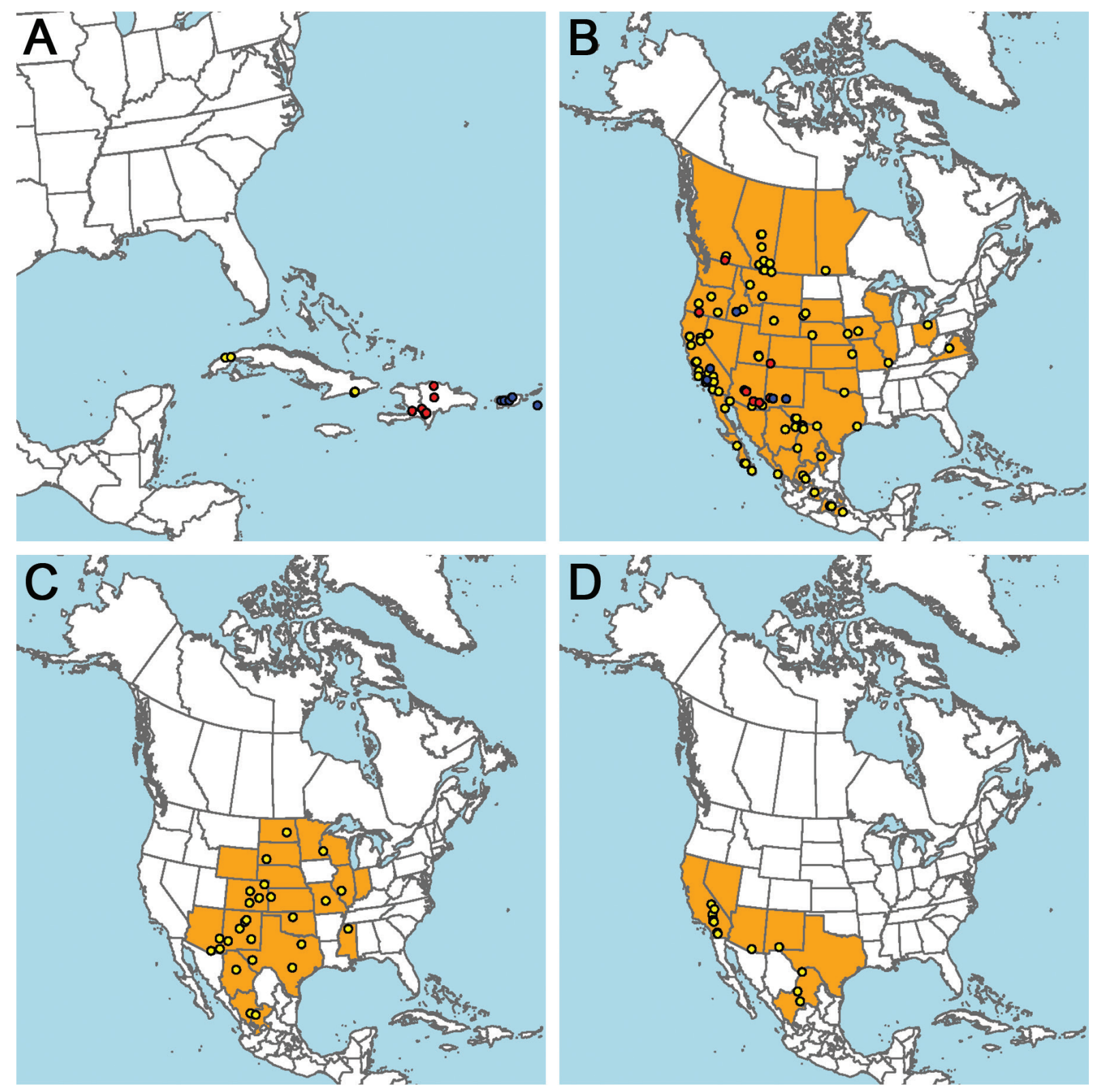

Fig. 4. Species distribution maps. A. Brachymelecta alayoi (Michener, 1988) (yellow circles), B. haitensis (Michener, 1948) (red circles), and B. tibialis (Fabricius, 1793) (blue circles). B. B. californica (Cresson, 1878) (red and blue circles indicate barcoded specimens assigned BINs BOLD:AAC6481 and BOLD:AAC6482, respectively). C. B. interrupta (Cresson, 1872). D. B. larreae (Cockerell, 1900). Known localities based on studied material and literature records are indicated by yellow circles except where indicated otherwise; subnational entities colored in orange indicate known/published provincial/ state records. 


\section{Material examined}

\section{Primary type material}

CANADA • +, M. sladeni holotype; British Columbia, Summerland; 9 Aug. 1916; F.W.L. Sladen leg.; CNC 652.

USA • $\widehat{O}$, M. californica lectotype; California; "H. Edwards, Behrens"; ANSP $2292 \cdot \odot$, , P. pasadenensis holotype; Pasadena, Los Angeles County; 30 Apr. 1909; F. Grinnell Jr. leg.; CAS 15525 • đ̃ , M.? mucida holotype; Nevada; H.K. Morrison leg.; ANSP 2294 • + , M. miranda holotype; South Dakota, Rapid City; J.T. Aldrich leg.; ANSP 10129.

\section{Secondary type material}

CANADA • 1 क, M. sladeni paratype; British Columbia, Westbank; 20 Jul. 1919; E.R. Buckell leg.; CNC 891698.

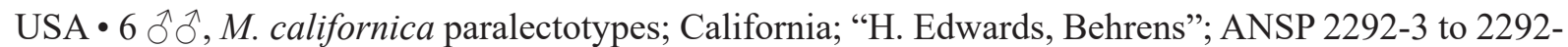
8・1 +, M. miranda paratype; South Dakota, Custer; J.T. Aldrich leg.; ANSP 10129.
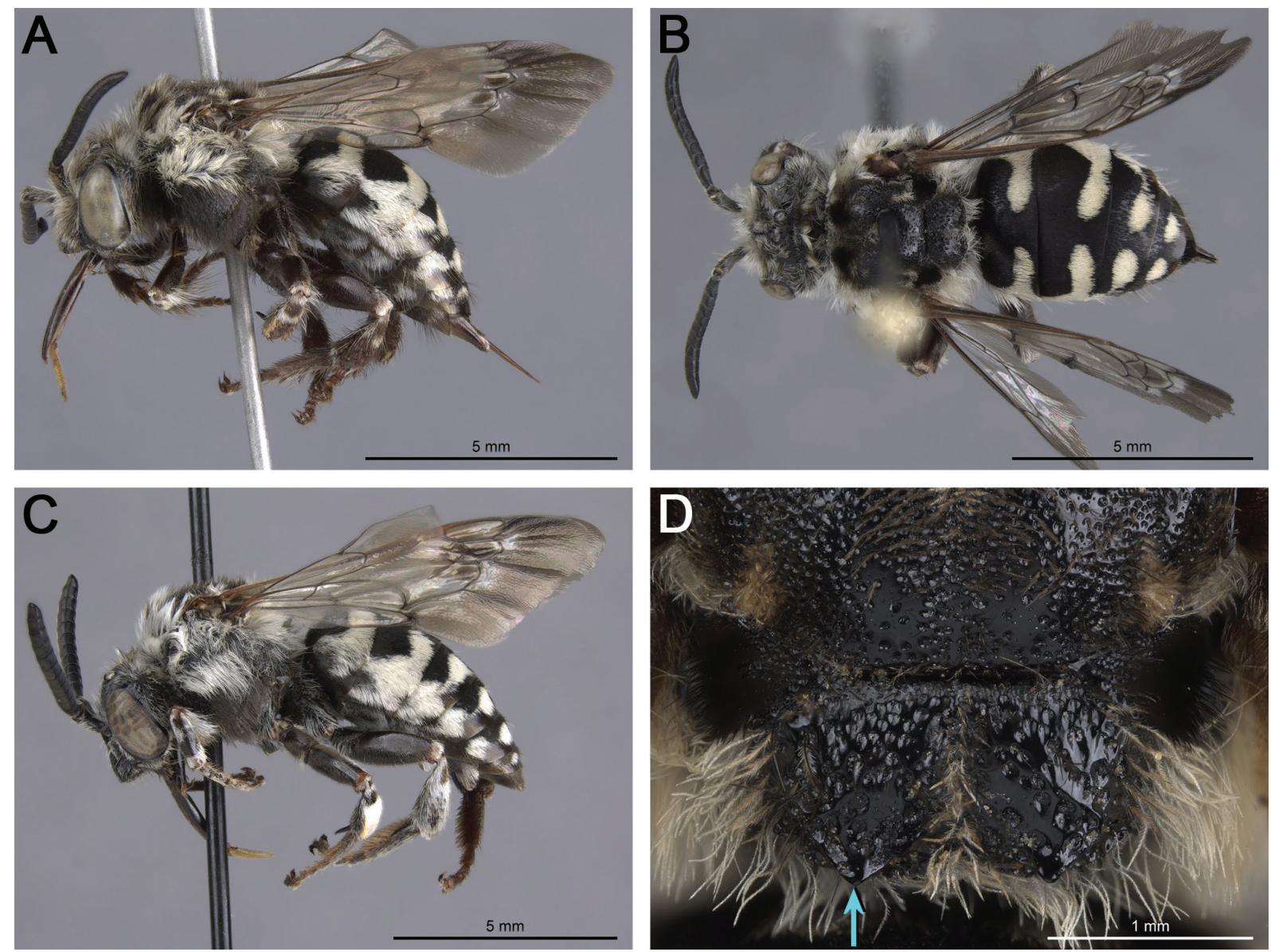

Fig. 5. Brachymelecta californica (Cresson, 1878). A. Female (PCYU PYU-2276), habitus, lateral view. B. Female (BOLD sample ID: CCDB-34570 A07; PCYU), habitus, dorsal view. C. Male (BOLD sample ID: CCDB-34570 A11; PCYU), habitus, lateral view. D. Female (PCYU PYU-2391), axillae and mesoscutellum, dorsal view (blue arrow indicates one of two short, mammiform tubercles of mesoscutellum). 
DNA barcoded material

Available. Two BINs:

BOLD:AAC6481. Specimens examined and sequenced:

CANADA - 1 \%; British Columbia, White Lake (Okanagan Falls), Regional District of OkanaganSimilkameen; $49.3028^{\circ}$ N, $119.6286^{\circ}$ W; 17 Aug. 2008; L.R. Best leg.; BOLD sample ID: LRBBC1015; PCYU LRB-445.

USA 1 ; ; Arizona, Catalina Hwy (Santa Catalina Mountains), Pima County; $32.3636^{\circ} \mathrm{N}, 110.7128^{\circ} \mathrm{W}$; 29 May 2015; T.M. Onuferko leg.; BOLD sample ID: CCDB-34570 A09; PCYU • 1 क; Arizona, Catalina Hwy (Santa Catalina Mountains), Pima County; $32.3242^{\circ}$ N, $110.7064^{\circ}$ W; 10 May 2016; T.M. Onuferko leg.; BOLD sample ID: CCDB-34570 B02; PCYU • 1 đ;; Arizona, Near Desert Botanical Garden (Phoenix), Maricopa County; 31 May 2018; T.M. Onuferko leg.; BOLD sample ID: CCDB34570 A11; PCYU • 1 \% ; Arizona, SE of Willcox, Cochise County; $32.2350^{\circ} \mathrm{N}, 109.7785^{\circ} \mathrm{W} ; 30$ Aug. 2016; L. Packer leg.; BOLD sample ID: CCDB-34570 A05; PCYU • 1 q; Colorado, Cortez, Montezuma County; $37.3570^{\circ}$ N, $108.5900^{\circ}$ W; 27 Jul. 2012; J. Gibbs and C.S. Sheffield leg.; BOLD sample ID: CCDB-06707 F05; PCYU PCYU-GS-07:870 • 1 क; New Mexico, NM 15 (16.7 mi N of Silver City), Gila National Forest; $32.9462^{\circ} \mathrm{N}, 108.1978^{\circ}$ W; 5 Sep. 2015; R.R. Ferrari leg.; BOLD sample ID: CCDB-34570 A03; PCYU • 1 q; Oregon, Hwy 140, Klamath County; $42.2132^{\circ}$ N, $121.8306^{\circ}$ W; 2 Jul. 2007; J. Gibbs and C.S. Sheffield leg.; BOLD sample ID: 07-OR-1265; PCYU PYU-2308.

BOLD:AAC6482. Specimens examined and sequenced:

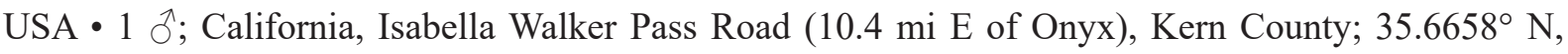
$118.0386^{\circ}$ W; 12 Sep. 2015; T.M. Onuferko and S. Tessier leg.; BOLD sample ID: CCDB-34570 A01; PCYU • 1 \&; California, Near Descanso Gardens (La Cañada Flintridge), Los Angeles County; 25 May 2018; T.M. Onuferko leg.; BOLD sample ID: CCDB-34570 A07; PCYU • 1 \%; Idaho, Hwy 20 (20 km E of Mountain Home), Elmore County; $43.2842^{\circ}$ N, $115.4765^{\circ}$ W; 27 May 2007; J. Gibbs and C.S. Sheffield leg.; BOLD sample ID: 07-ID-1195; PCYU PYU-1208 • 1 q; New Mexico, Cloudcroft, Otero County; 32.9574 ${ }^{\circ}$ N, $105.7447^{\circ}$ W; 11 Aug. 2007; J. Gibbs and C.S. Sheffield leg.; BOLD sample ID: 07-NM2108; PCYU PYU-3519 • 1 ``; New Mexico, Hwy 152, Sierra County; $32.8949^{\circ}$ N, $107.7399^{\circ}$ W; 12 Aug. 2007; J. Gibbs and C.S. Sheffield leg.; BOLD sample ID: 07-NM-2109; PCYU PYU-3530.

\section{Non-barcoded material}

CANADA • 1 q; Alberta, Bull Trail Park (Lethbridge); $49.6933^{\circ}$ N, $112.8703^{\circ}$ W; 9 Jul. 2020; M. Buck leg.; RAM PMAE00156227 • 1 +; Alberta, Bull Trail Park (Lethbridge); $49.6964^{\circ} \mathrm{N}, 112.8714^{\circ} \mathrm{W}$; $9 \mathrm{Jul}$. 2020; M. Buck leg.; RAM PMAE00156145 • 6 + ; Alberta, Dry Island Buffalo Jump Provincial Park, Kneehill County; $51.9417^{\circ} \mathrm{N}, 112.9481^{\circ} \mathrm{W}$; 20 Jul. 2016; M. Buck leg.; RAM • 1 \%; Alberta, Dry Island

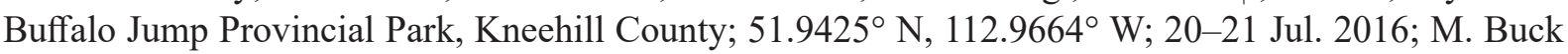

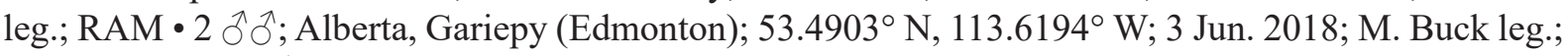
RAM $\bullet 2$ 아, 1 ; ; same collection data as for preceding; 16 Jun. 2018; RAM • 1 q; Alberta, Helen Schuler Nature Reserve (Lethbridge); 49.7008 ${ }^{\circ}$ N, 112.8603 ${ }^{\circ}$ W; 13 Jul. 2020; M. Buck leg.; RAM PMAE00156567 • 1 क; Alberta, Lethbridge; 11 Aug. 1944; O. Peck leg.; CNC 891702 • 1 q; Alberta, Milk River (Weir Bridge), Warner County; $49.1042^{\circ} \mathrm{N}, 111.7000^{\circ} \mathrm{W} ; 24$ Jul. 2020; M. Buck leg.; RAM PMAE00157991 • 2 우; Alberta, Near Capilano Foot Bridge (Edmonton); $53.5642^{\circ} \mathrm{N}, 113.4192^{\circ} \mathrm{W}$; 17 Jun. 2018; M. Buck leg.; RAM • 1 ○; Alberta, Redcliff; 50.0539 ${ }^{\circ}$ N, $110.7758^{\circ}$ W; 28 Jun. 2017; M. Buck leg.; RAM • 1 क; same collection data as for preceding; 29 Jun. 2017; RAM 11 ○ं; Alberta, Scandia; 21 Jul. 1928; F.W. Barnes leg.; CNC 891692 • 1 q; Alberta, Terwillegar Park (Edmonton);

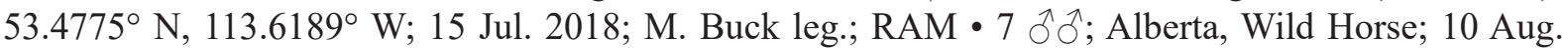
1927; H.E. Gray leg.; CNC 891691, 891693 to 891696, 891704, 899632 • 2 qo+; Alberta, Writing-on- 
Stone Provincial Park (Coffin Bridge), Warner County; 49.1033º N, $111.8903^{\circ}$ W; 15 Jul. 2020; M. Buck leg.; RAM PMAE00156796, PMAE00156797 • 1 q; Alberta, Writing-on-Stone Provincial Park (Davis Coulee), Warner County; 49.0708 ${ }^{\circ} \mathrm{N}, 111.6281^{\circ} \mathrm{W} ; 22$ Jul. 2020; M. Buck leg.; RAM PMAE00157617 - 2 우; Manitoba, Treesbank, Municipality of Glenboro-South Cypress; $49.6350^{\circ} \mathrm{N}, 99.6040^{\circ} \mathrm{W} ; 1 \mathrm{Jul}$. 2018; Manitoba Conservation Data Centre leg.; WRME JBWM0415546, JBWM0415590.

MEXICO • 1 đ̇; Baja California Sur, San Hilario; 5 Nov. 1968; E.L. Sleeper and F.J. Moore leg.; CAS - 1 J ; Baja California Sur, Santa Victoria La Laguna; 20 or 23 Oct. 1968; E.L. Sleeper and F.J. Moore

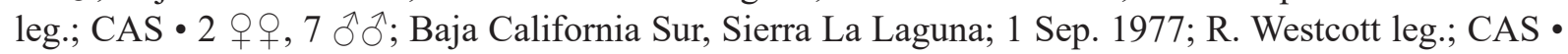
1 क; Distrito Federal, Xochimilco; 18 Aug. 1962; H.E. Milliron; CNC 394149 • 1 P; Sinaloa, Mazatlán; Feb. ??53; CAS • 1 §̊; Sonora, Gran Desierto de Altar; 20 Mar. 2018; H. Knight leg.; CSUC.

USA • 1 đ̇; Arizona, 4 mi E of Willcox, Cochise County; 27 Aug. 2007; G. Rowe leg.; PCYU • 1 क ; Arizona, East Moonlight Road, Cochise County; $32.2388^{\circ}$ N, $109.7651^{\circ}$ W; 14 Aug. 2007; J. Gibbs and C.S. Sheffield leg.; PCYU PYU-5972 • 1 q; Arizona, Rustler Park (Chiricahua Mountains), Cochise County; $31.9039^{\circ}$ N, 109.2792 ${ }^{\circ}$ W; 11 Aug. 2007; M. Buck leg.; DEBU debu00291918 • 2 우; California, $13 \mathrm{mi} \mathrm{W}$ of Coalinga; 30 Jul. ??59; CNC 891731, 891732 • 1 क; California, $3 \mathrm{mi} \mathrm{N}$ of Coalinga; 29 Jul. 1954; F.M. Hull leg.; CNC 891744 5 우; California, 28 km NE of Foresthill (Tahoe National Forest), Placer County; $39.1856^{\circ}$ N, $120.6058^{\circ}$ W; 8 Jun. 2009; M. Buck leg.; RAM • 1 o; California, $30 \mathrm{~km}$ NE of Foresthill (Tahoe National Forest), Placer County; $39.1894^{\circ} \mathrm{N}, 120.5756^{\circ} \mathrm{W}$; 8 Jun. 2009; M. Buck leg.; RAM • 1 क ; California, Antioch; Oct. 1938; J.A. Downes leg.; CNC 846577 - 2 ơ $^{\top}$; California, CA-20 $1.7 \mathrm{~km} \mathrm{~W}$ of Jct CA-16, Colusa County; 39.0133 ${ }^{\circ} \mathrm{N}, 122.3792^{\circ} \mathrm{W}$; 9 Jun.

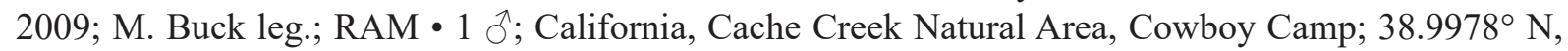
122.3558 W; 9 Jun. 2009; M. Buck leg.; RAM • 1 क ; California, Helendale, San Bernardino County; 16 May 1955; W.R. Richards leg.; CNC 891690 • 1 q; same collection data as for preceding; 21 May 1955; W.R.M. Mason leg.; CNC 891736 • 1 q; California, Mill Creek, San Bernardino Mountains; 31 Aug. 1930; C.D. Michener leg.; CNC 891705 • 1 क; California, Mojave; 15 Oct. ??25; R. Hopping leg.; CNC 891703 • 3 q 우 California, Mojave Desert; 15 Oct. 1925; J.M. Swaine leg.; CNC 891697, 891700 to $891701 \cdot 1$ o; California, Peavine Ridge Road, El Dorado County; $38.7964^{\circ} \mathrm{N}, 120.4297^{\circ} \mathrm{W}$;

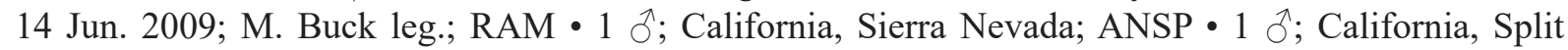
Mountain (Anza-Borrego Desert State Park), San Diego County; 1 Apr. 1955; J.E.H. Martin leg.; CNC 891713 • 1 đ̊; California, UCR Botanic Gardens (Riverside), Riverside County; 26 Jun. 1984; S.L. Heydon leg.; INHS 387473•1 O; Idaho, Hwy 75 (5 km N of Ketchum), Blaine County; $43.7630^{\circ} \mathrm{N}$,

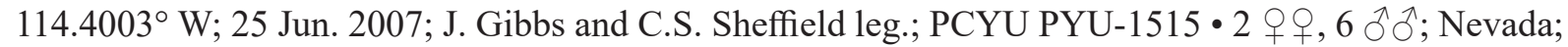
ANSP 1 ơ; Nevada, Pyramid Lake (south shore), Washoe County; $39.8444^{\circ} \mathrm{N}, 119.4400^{\circ} \mathrm{W}$; 13 Jun. 2009; M. Buck leg.; RAM • 1 क; New Mexico, Hwy 152, Sierra County; $32.8949^{\circ}$ N, $107.7399^{\circ}$ W; 12 Aug. 2007; J. Gibbs and C.S. Sheffield leg.; PCYU PYU-2391 • 1 đ̊; New Mexico, Hwy 152, Sierra County; $32.8949^{\circ}$ N, $107.7399^{\circ}$ W; 12 Aug. 2007; J. Gibbs and C.S. Sheffield leg.; PCYU PYU-2392 - 1 ○; Oregon, Fish Lake (near Steens Mountain), Harney County; $42.7419^{\circ} \mathrm{N}, 118.6461^{\circ} \mathrm{W} ; 6$ Aug. 2005; J. and A. Skevington leg.; CNC 891706 • 1 q; Oregon, Hwy 140, Klamath County; $42.2132^{\circ}$ N, $121.8306^{\circ}$ W; 2 Jul. 2007; J. Gibbs and C.S. Sheffield leg.; PCYU PYU-2292 • 3 q ${ }^{\circ}$; Oregon, Hwy 26, Crook County; $44.4721^{\circ}$ N, $120.4197^{\circ}$ W; 28 Jun. 2007; J. Gibbs and C.S. Sheffield leg.; PCYU PYU-2111, PYU-2676, PYU-2682 • 1 9; Oregon, Hwy 97, Klamath County; $42.1224^{\circ} \mathrm{N}, 121.8273^{\circ} \mathrm{W}$; 2 Jul. 2007; J. Gibbs and C.S. Sheffield leg.; PCYU PYU-2276 • 1 đo; Texas, $23 \mathrm{mi} \mathrm{W}$ of Fort Davis; 1 Jun. 1959; F. McAlpine leg.; CNC 891707• 1 क; Texas, Big Bend National Park, Boquillas; 17 May 1959; W.R.M. Mason leg.; CNC 891724 • 3 ô $\widehat{\jmath}$; Texas, Big Bend National Park, Dagger Flats; 11 May 1959; W.R.M. Mason leg.; CNC 891721, 891722, 891727 • 1 q; Texas, Big Bend National Park, Nine Point Draw; 15 May 1959; W.R.M. Mason leg.; CNC 891725• 1 ð̊; Texas, Big Bend National Park, Panther Junction; 16 May 1959; J.F. McAlpine leg.; CNC 891723・ 1 o ; Texas, Devils River, Del Rio; 26 Apr. 1959; J.F. McAlpine leg.; CNC 891715 • 1 q; Texas, Dickinson, Galveston County; Jun. 1929; 
F.M. Hull leg.; CNC 891734 • 1 9; Texas, Las Burras Canyon at FM-170, Presidio County; $29.3903^{\circ}$ N, 104.1428 W; 28 Apr. 2019; M. Buck leg.; RAM • 1 गे; Texas, near Point of Rocks (10 mi W of Fort Davis); 29 May 1959; F. McAlpine leg.; CNC 891720 • 1 ふै; Utah, 24 mi S of Hanksville; 18 Jul. 1968; J.E.H. Martin leg.; CNC 891733 • 3 ồ; same collection data as for preceding; 23 Jul. 1968; CNC 891728 to 891730 • 1 đ̂; Utah, Fairview Ranch (13 mi S of Hanksville); 2-3 Aug. 1968; H.F. Howden leg.; CNC 891726.

\section{Redescription}

\section{Male}

Measurements. Length $9.9 \mathrm{~mm}$; ITW $2.6 \mathrm{~mm}$; head length $2.7 \mathrm{~mm}$; head width $3.3 \mathrm{~mm}$; fore wing length $9.2 \mathrm{~mm}$.

InTEGUMent COLORATION. Dark brown to black except as follows. Mandible with apical third golden yellow. Mandible with middle third, legs, excluding black tibial spurs, and much of metasoma reddish brown. Tegula amber. Fore wing dusky apically, with most cells and around third submarginal crossvein and second recurrent vein subhyaline. Hind wing hyaline, slightly dusky beyond venation.

PuBESCENCE. Face with hairs densest around antennal socket, predominantly off-white but dark brown/ gray in lower paraocular area. Clypeus bare in M. californica lectotype, with many hairs rubbed off, but with dense hairs in multiple paralectotypes and non-type specimens and M.? mucida holotype. Upper paraocular and frontal areas and vertexal area (except along preoccipital ridge) mostly exposed. Head with dense, erect hairs along preoccipital ridge off-white except for pair of dark brown/gray patches, each behind lateral ocellus and in front of paramedian band. Genal beard hairs predominantly off-white. Mesoscutum sparsely hairy except densely hairy anteriorly and along margins, with hairs predominantly off-white except for dark brown/gray paramedian band. Axilla with conspicuous patch of black hairs. Mesoscutellum, metanotum, and propodeum with erect, predominantly off-white hairs. Mesopleuron with upper half covered in dense, off-white hairs greatly obscuring underlying integument, with large circular patch of sparser, dark brown/gray to black hairs not obscuring underlying integument occupying much of ventrolateral half, and with predominantly off-white hairs somewhat obscuring underlying integument ventrally around margin. Legs, from coxae to tarsi, with appressed and erect off-white hairs, ventrally with golden-yellow hairs. Profemur with posteroventral fringe of dense, off-white hairs. Protibia and metatibia each with two bands or spots of short, appressed off-white hairs, one in basal half and one on apical margin. Mesotibia with patch of very dense short, appressed off-white hairs, occupying nearly entire dorsal surface. T1-T5 with well-defined, medially interrupted apical fasciae, each with pair of lobe-like anterolateral extensions on each side with erect among appressed off-white hairs. T3-T5 each with fascia more widely removed from apical margin and laterally narrowed or interrupted, mesad the inner pair of anterolateral extensions. T6 almost completely retracted under T5 in M. californica lectotype, but with paired patches of off-white tomentum in paralectotype and multiple non-type specimens. Exposed metasomal sterna mainly with short, appressed off-white hairs. S4 and S5 each with apical/subapical fringe of dense, long, curved, coppery to silvery hairs.

SURFACE SCULPTURE. Labrum sparsely punctate (many $\mathrm{i}>2 \mathrm{~d}$ ). Clypeus with upper half densely punctate (most $i \leq 1 \mathrm{~d}$ ), lower half more sparsely punctate (many $\mathrm{i}>1 \mathrm{~d}$ ). Small impunctate shiny spot lateral to lateral ocellus. Mesoscutum and mesoscutellum with coarser punctures than metasomal terga. Mesoscutum densely punctate (most $\mathrm{i} \leq 1 \mathrm{~d}$ ) except for small impunctate shiny spot between paramedian band and parapsidal line and in posterior half between midline and parapsidal line, where more sparsely punctate (many $\mathrm{i}>1 \mathrm{~d}$ ). Mesoscutellum with coarser punctures than mesoscutum, densely punctate (most $i \leq 1 \mathrm{~d}$ ) except medially (between pair of mammiform tubercles), where somewhat sparsely punctate $(i \leq 2 \mathrm{~d})$. Mesopleuron densely punctate (most $\mathrm{i}<1 \mathrm{~d}$ ) but with most interspaces well-defined, shining. Discs of 
metasomal terga with punctures very fine, sparse (most $i>1 d$ ), interspaces dull due to tessellate surface microsculpture.

STRUCTURE. Mandible tridentate, with small inner tooth approximately $1 / 3$ length of mandible from base and equally small inner tooth approximately $1 / 3$ length of mandible from apex as well as usual large apical tooth (rutellum) (difficult to see in M. californica lectotype because mandibles closed; described from paralectotypes, non-type specimens, and M.? mucida holotype). Maxillary palpus with four palpomeres (mouthparts not extended in M. californica lectotype; described from paralectotypes and non-type specimens). Scape with greatest length $1.9 \times$ greatest width. F2 wider than long (L/W ratio $=0.8$ ). Mesoscutellum strongly bigibbous, with pair of short, apically flattened mammiform tubercles. Lateral surface of propodeum posterior to spiracle with rugose crescent ridge, strongly carinate above and joining anterior lip of spiracle. Fore wing with two or three submarginal cells (in M. californica lectotype, second submarginal crossvein of left fore wing incomplete, that of right fore wing complete). $\mathrm{T} 7$ distinctly notched medially between pair of small medioapical projections.

\section{Female}

Description as for male except for usual secondary sexual characters and as follows: scape longer, with greatest length $2.3 \times$ greatest width; mesotibia dorsally with off-white hairs forming small band or spot, nearer the base than apex; T6 with narrow, V-shaped but apically rounded pygidial plate with median longitudinal ridge and without patches of off-white tomentum; S4 and S5 apically/subapically without dense, long, curved, coppery to silvery hairs.

\section{Distribution}

Brachymelecta californica is the most widely distributed member of its genus, ranging from Western Canada to southern Mexico west of the Isthmus of Tehuantepec (Fig. 4B). Although this species has been recorded from the Eastern United States (Mitchell 1962), it is much more common in the west, with few known records east of the Mississippi River. This is the only species of Brachymelecta known to occur in Canada.

\section{Ecology}

\section{Host records}

Brachymelecta californica has been reared from the nests of multiple species of Anthophora, including A. abrupta Say, 1837, A. bomboides stanfordiana Cockerell, 1904 (a junior synonym of $A$. bomboides Kirby, 1838), A. edwardsii Cresson, 1878, A. linsleyi Timberlake, 1941, A. neomexicana (a junior synonym of $A$. bomboides), A. occidentalis Cresson, 1869, and A. urbana Cresson, 1878 (Hicks 1926; Linsley \& McSwain 1942; Linsley 1943; Torchio \& Trostle 1986; Rozen 1991). Additionally, Orr et al. (2016) documented the emergence of adults of this cuckoo bee species from nests of A. pueblo Orr, 2016.

\section{Floral records}

Although known to visit a very large variety of flowers, in a survey by P.H. Timberlake Brachymelecta californica was found to be most abundant on flowers of various Asteraceae (Linsley 1939). His floral records, based on collections at a single locality in Riverside, California, USA, are as follows: Brassica incana Ten. (Brassicaceae), Coreopsis lanceolata L. (Asteraceae), Croton setigerus Hook. (Euphorbiaceae) (as Eremocarpus setigerus), Cryptantha Lehm. ex G. Don (Boraginaceae), Duranta erecta L. (Verbenaceae) (as D. plumieri), Encelia farinosa A.Gray ex Torr. (Asteraceae), Ericameria palmeri (A.Gray) H.M.Hall (Asteraceae), Eriogonum fasciculatum Benth. (Polygonaceae), E. gracile Benth., Gutierrezia californica (DC.) Torr. \& A.Gray (Asteraceae), Hemizonia paniculata A.Gray (Asteraceae), Lepidospartum squamatum (A.Gray) A.Gray (Asteraceae), Marrubium L. (Lamiaceae), Phacelia distans Benth. (Boraginaceae), Pluchea camphorata (L.) DC. (Asteraceae), Senecio flaccidus 
var. douglasii (DC.) B.L.Turner \& T.M.Barkley (Asteraceae) (as S. douglasii), and Trichostema lanceolatum Benth. (Lamiaceae).

Hurd (1953) reported this species on Eysenhardtia polystachya (Ortega) Sarg. (Fabaceae) and Hurd et al. (1980) on Helianthus annuus L. (Asteraceae) and H. gracilentus A.Gray. Floral records from data contributors to Discover Life (Ascher \& Pickering 2020) compiled by J. Pickering are as follows: Adenostoma Hook. \& Arn.(Rosaceae), Aster L.(Asteraceae), Astragalus L.(Fabaceae), Baileya Harv. \&A. Gray ex A. Gray (Asteraceae), Bebbia juncea (Benth.) Greene (Asteraceae), Chrysothamnus viscidiflorus (Hook.) Nutt. (Asteraceae), Cirsium vulgare (Savi) Ten. (Asteraceae), Clarkia Pursh (Onagraceae), Ericameria nauseosa (Pall. ex Pursh) G.L.Nesom \& G.I.Baird, Ericameria parryi (A.Gray) G.L.Nesom \& G.I.Baird, Eriodictyon californicum (Hook. \& Arn.) Decne. (Boraginaceae), Gaillardia Foug. (Asteraceae), Grindelia Willd. (Asteraceae), Gutierrezia sarothrae (Pursh) Britton \& Rusby, Horkelia Cham. \& Schltdl. (Rosaceae), Larrea tridentata (Sessé \& Moc. ex DC.) Coville (Zygophyllaceae), Lonicera L. (Caprifoliaceae), Lorandersonia linifolia (Greene) "Urbatsch, R.P.Roberts \& Neubig" (Asteraceae) (as Chrysothamnus linifolius), Melilotus officinalis (L.) Pall. (Fabaceae), Melilotus albus Medik. (as M. alba), Monardella Benth. (Lamiaceae), Palafoxia arida B.L.Turner \& M.I.Morris (Asteraceae), Penstemon cyananthus Hook. (Plantaginaceae), Petrophytum caespitosum (Nutt. ex Torr. \& A.Gray) Rydb. (Rosaceae) (as Petrophyton caespitosum), Phacelia crenulata Torr. ex S. Watson, P. hastata Douglas ex Lehm., Salsola kali L. (Amaranthaceae), Salvia L. (Lamiaceae), Sedum stenopetalum Pursh (Crassulaceae), Senecio flaccidus Less. (as S. longilobus), Solidago L. (Asteraceae), Sphaeralcea orcuttii Rose (Malvaceae), Stanleya pinnata (Pursh) Britton (Brassicaceae), Syrmatium decumbens (Benth.) Greene (Fabaceae) (as Lotus nevadensis), Tamarix L. (Tamaricaceae), Tetradymia DC. (Asteraceae), and Verbesina encelioides (Cav.) Benth. \& Hook.f. ex A.Gray (Asteraceae). Images on iNaturalist (https://www.inaturalist.org) show this species visiting Echinacea purpurea (L.) Moench (Asteraceae) and images on BugGuide (https://bugguide.net) show it visiting Achillea millefolium L. (Asteraceae), Baccharis salicina Torr. \&A.Gray (Asteraceae), Centaurea nigra L. (Asteraceae), Chorizanthe staticoides Benth. (Polygonaceae), Cirsium wheeleri (A.Gray) Petr., Echinacea pallida (Nutt.) Nutt., Erodium cicutarium (L.) L’Hér. (Geraniaceae), Lantana L. (Verbenaceae), Osteospermum L. (Asteraceae), and Rosmarinus officinalis L. (Lamiaceae). Labels of examined voucher specimens further indicate that this species has been collected from Chilopsis D. Don (Bignoniaceae) and Sisymbrium loeselii L. (Brassicaceae).

\section{Remarks}

The holotype of M.? mucida generally agrees with the diagnosis for B. californica provided herein. Additionally, examination of the dissected terminalia revealed that the hidden sterna and genitalia of the M.? mucida holotype fall within the range of morphological variation observed among the five other specimens of $B$. californica that were dissected, including two $M$. californica paralectotypes. One notable feature unique to B. californica and shared by all dissected specimens (including the M.? mucida holotype) is that $\mathrm{S} 7$ has long setae along its entire posterior margin (Fig. 6A-B). By contrast, in B. haitensis S7 has a tuft of dense setae posteromedially (Fig. 6C) and in B. interrupta and B. larreae S7 has only a few short setae posteromedially (Fig. 6D-E). In the M.? mucida holotype, S8 is apparently damaged/split anteromedially, and thus somewhat distorted, but otherwise closely resembles that of other specimens of B. californica (Fig. 6A-B). No differences were found in the morphology of the genital capsule between the M.? mucida holotype and other specimens of B. californica, but the genitalia are also highly similar among the six species in the genus.

The only diagnostic features that seemingly support B. mucida as a separate species include the presence of two submarginal cells on each of the two fore wings (Fig. 7A, C) and pale tomentum that obscures almost the entire dorsal surface of the metasoma (Fig. 7A-B). However, specimens of B. californica in which both fore wings have two submarginal cells are known (e.g., Fig. 8A), and in multiple paralectotypes 

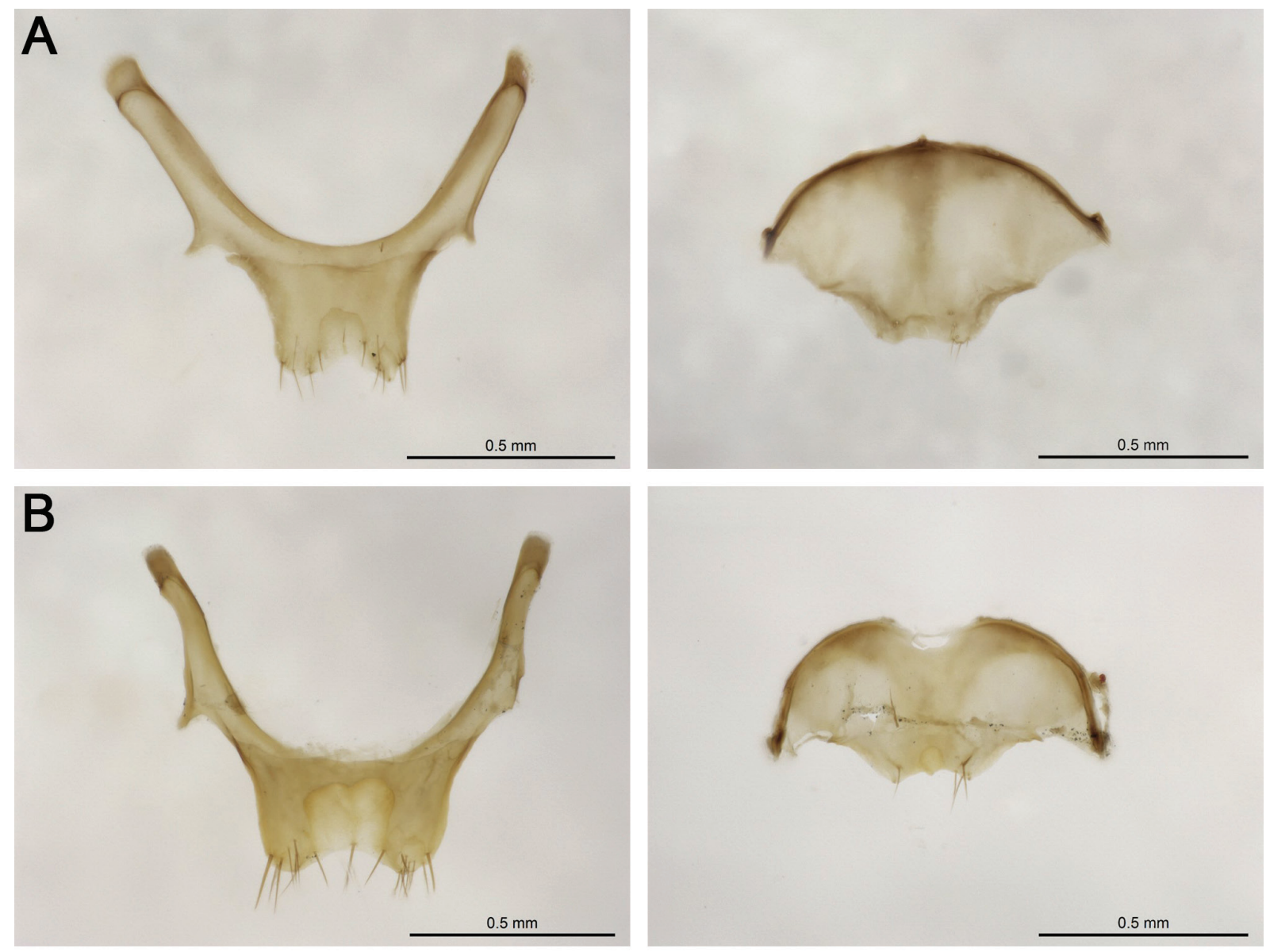

\section{C}
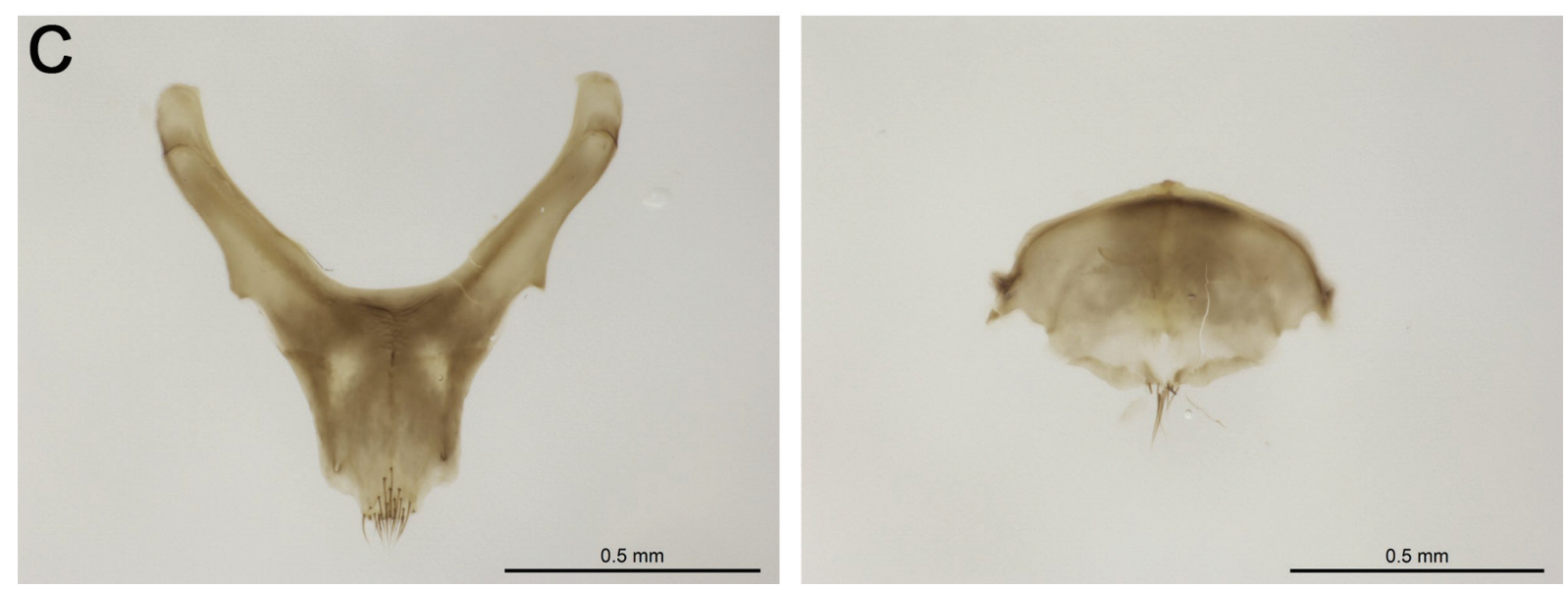

Fig. 6A-C (continued on next page). Dissected male S7 (left) and S8 (right), ventral view (with anterior ends oriented toward the top). A. Brachymelecta californica (Cresson, 1878) (photo of Melecta californica paralectotype (ANSP 2292-5)). B. B. californica (photo of M.? mucida holotype (ANSP 2294) (herein synonymized under B. californica)) (S8 appears to have been damaged/split anteromedially). C. B. haitensis (Michener, 1948) (JAG). 
(Fig. 8B-C) and even the lectotype of M. californica, the second submarginal crossvein is incomplete in one wing, resulting in two submarginal cells, and complete in the other, resulting in three. Recently, a specimen of $B$. californica was collected that, like the M.? mucida holotype, has abundant pale tomentum covering the surface of the metasomal terga (Fig. 8D). This specimen has been barcoded (BOLD sample ID: CCDB-34570 A03) and its sequence assigned to the BIN BOLD:AAC6481. However, the hairs on each tergum are not uniformly dense, such that distinct apical fasciae are still visible on T1-T5 (Fig. 8D). Close examination of the dorsal surface of the metasoma of the M.? mucida holotype also revealed the presence of an apical fascia on each exposed metasomal tergum comprised of short, appressed, branched pale hairs slightly denser than those covering the disc (Fig. 7A-B). Hence, the anatomical features previously considered to be diagnostic for $B$. mucida fall within the range of variation observed within $B$. californica, so the former is herein synonymized under the latter.

In the original description, Fox (1893) described Melecta miranda (now an established junior synonym of B. californica, Hurd \& Linsley 1951) from an unindicated number of specimens from Rapid City and Custer, South Dakota. In the ANSP entomological collection, one specimen from Rapid City, SD bears a red label that says "TYPE No. 10129" and is in the primary type collection whereas another from Custer, SD bears a blue label that says "PARATYPE 10129" (the catalog number is the same for both specimens) and is in the regular collection. Although in the original description it is not indicated which specimen is the primary type, that which bears the red type label, which does not say paratype, is
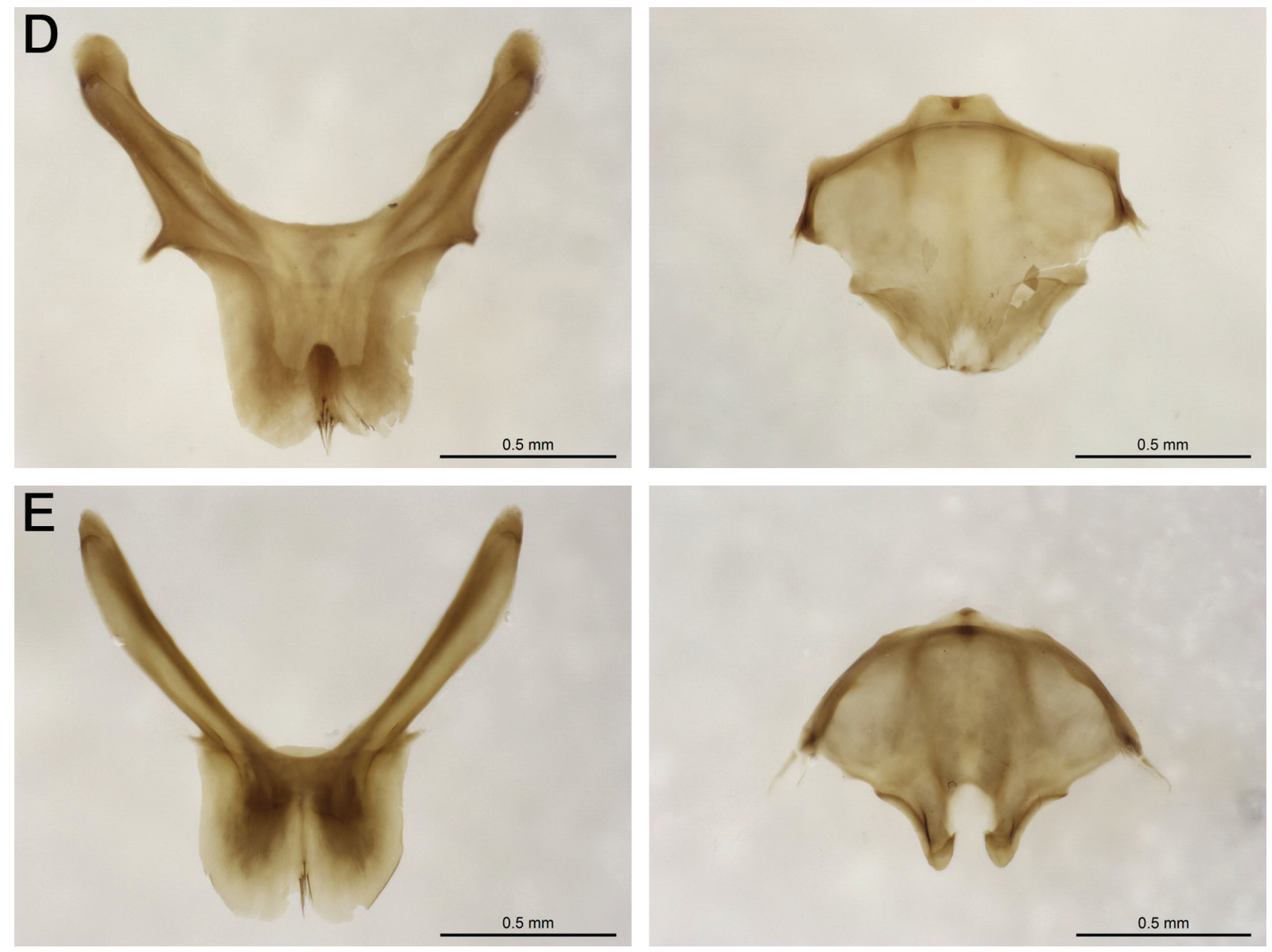

Fig. 6D-E (continued). Dissected male S7 (left) and S8 (right), ventral view (with anterior ends oriented toward the top). D. Brachymelecta interrupta (Cresson, 1872) (ANSP). E. B. larreae (Cockerell, 1900) (CNC 891710). 
presumed to be the holotype as there is no indication that the species was described from more than the two specimens.

Barcoded specimens identified as B. californica (agreeing with the diagnosis for the species presented herein) were assigned one of two BINs (vide supra), which are each other's nearest neighbors. Despite a barcode sequence divergence of $4.9 \%$ between the two MOTUs (see Supp. file 3: fig. S1), which are largely sympatric (see Fig. 4B), no consistent morphological differences were found (including in the dissected terminalia of two males that were assigned separate BINs, which fall within the range of morphological variation observed within this species). In all barcoded specimens, both fore wings have three submarginal cells, so the MOTUs cannot be identified by the number of submarginal cells on the fore wing, which in B. californica may differ between the left and right sides of the same specimen anyway.

Melecta miranda, now synonymous with $B$. californica, was originally described as a separate species because of its larger body size (15.2 $\mathrm{mm}$ in length, excluding the protruding sting), but barcoded specimens assigned separate BINs exhibit much overlap in body length (9.9-12.1 mm for BOLD BIN AAC6481 vs 9.1-10.5 mm for AAC6482), so body size could not be linked to haplotype. Pseudomelecta pasadenensis, now synonymous with B. californica, was described as separate from
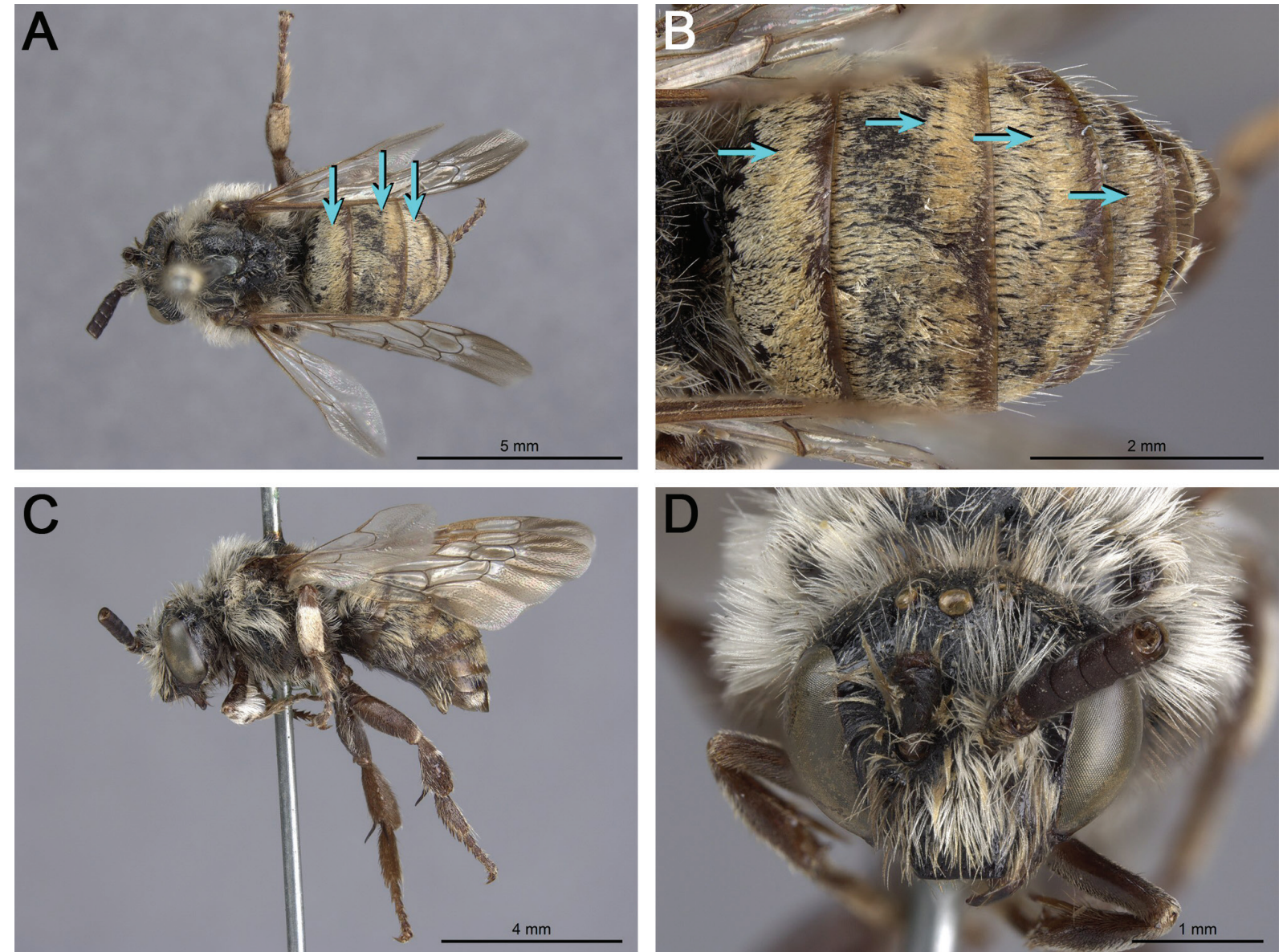

Fig. 7. Melecta? mucida holotype, $\widehat{O}$ (ANSP 2294) (herein synonymized under B. californica (Cresson, 1878)). A. Habitus, dorsal view (blue arrows indicate apical fasciae of metasomal terga). B. Metasoma, dorsal view (blue arrows indicate apical fasciae of metasomal terga) (pubescence appears to be matted from prior wetting). C. Habitus, lateral view. D. Head, frontal view. 
B. californica and M. miranda (as P. californica and P. miranda) on the basis of exhibiting tufts of black hair above the antennal sockets as well as in the lower paraocular area (Cockerell 1910), but barcoded specimens exhibiting this feature were divided between the two groups with separate BINs. Linsley (1939) further separated B. californica (as M. californica) from P. pasadenensis (as M. pasadenensis) by whether the T1 basal declivity has a fascia (said to be present in the former and absent in the latter). However, both haplotypes are represented by specimens in which T1 has a basal fascia. Viereck (1924) described $M$. sladeni as a new species superficially similar to $M$. miranda, the primary difference being that in the former the mammiform tubercles of the mesoscutellum are more similar to those of $M$. (now B.) interrupta. Having examined the primary types of M. interrupta, M. miranda, and $M$. sladeni, we conclude that the mesoscutella of the latter two are more similar to one another than either is to the mesoscutellum of the lectotype of $M$. interrupta. There also does not appear to be any consistent difference in the form of the mesoscutellum between specimens of the two haplotypes. Given
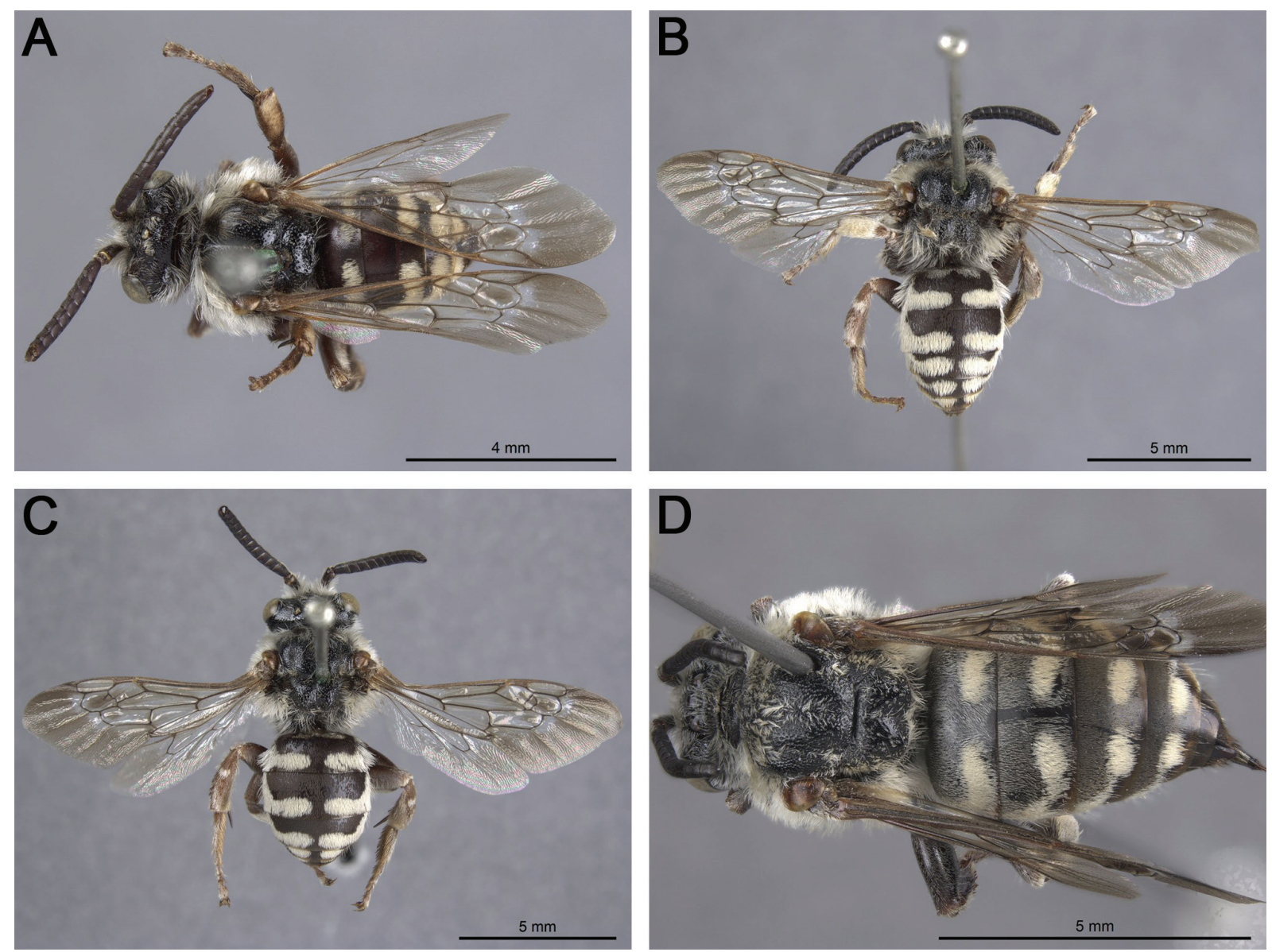

Fig. 8. Brachymelecta californica (Cresson, 1878), habitus, dorsal view. A. Male specimen (ANSP) from Nevada (bearing the same 'Nev' collection label as the Melecta? mucida holotype) in which both fore wings have two submarginal cells. B. M. californica paralectotype, $\widehat{\delta}$ (ANSP 2292-7) in which the left fore wing has three submarginal cells and the right has two, with the second submarginal cross vein incomplete. C. M. californica paralectotype, §̂ (ANSP 2292-4) in which the left fore wing has two submarginal cells and the right has three (note that in both fore wings, the second submarginal cross vein (incomplete on the left, complete on the right) originates from the first submarginal cross vein, not Rs as is typical for most bees, including this species). D. Female specimen (BOLD sample ID: CCDB-34570 A03; PCYU) in which the discs of the metasomal terga are (unusually for B. californica) covered in short, pale hairs, sparser than those comprising the apical fasciae. 
that the observed morphological variation is continuous and that it is possible for real species to have split (i.e., multiple) BINs (Ratnasingham \& Hebert 2013; see Ferrari 2017 for several examples within the bee genus Colletes Latreille, 1802 (Hymenoptera: Colletidae: Colletinae)), we recognize both series of barcoded specimens as a single species, $B$. californica, one in which intraspecific barcode sequence divergence is higher than might usually be expected among conspecifics.

Brachymelecta haitensis (Michener, 1948)

Figs 4A, 6C, 9A, 10-11, 12B, 17

Melecta haitensis Michener, 1948: $16\left({ }^{\Uparrow}\right)$.

\section{Proposed common name}

Hispaniolan digger-cuckoo bee.

\section{Diagnosis}

Unique within the genus to $B$. haitensis is that the T1-T3 fasciae of the male are complete (Fig. 9A). In all other species of Brachymelecta, at least the T2 and T3 fasciae (if present) (as well as any fasciae on the succeeding terga) are interrupted medially in males (Figs 8A-C, 9B) as well as females, except in aberrant specimens in which the metasoma is entirely covered in pale tomentum (see Fig. 7A-B). The following morphological features in combination (excluding any that are specific to the opposite sex of the one being diagnosed) can be used to tell B. haitensis apart from all other Brachymelecta except B. tibialis, a Puerto Rican/U.S. Virgin Islands species: the mesoscutum and mesoscutellum both have well-defined bands of pale hairs along the entire midline that connect and thus give the appearance of a single band (Figs 10B, D, 11); the mesoscutellum sometimes also has pale hairs laterally but the pubescence is otherwise dark brown or black, sparse, and does not obscure the underlying integument (Fig. 10D); and each mesotibia of the female dorsally has a large glabrous area between a submedial band of off-white to pale yellow hairs (nearer the base than apex) and a band on the apical margin (Fig. 10A). Apart from the difference in the T1-T3 fasciae of the male, B. haitensis may be separated from B. tibialis as follows. Whereas in B. tibialis the T1-T3 fasciae of the female are widely interrupted medially and the lighter hairs covering the head, mesosoma, and metasoma are off-white, in B. haitensis the T1-T3 apical fasciae of the female are only narrowly interrupted medially and the lighter hairs covering the
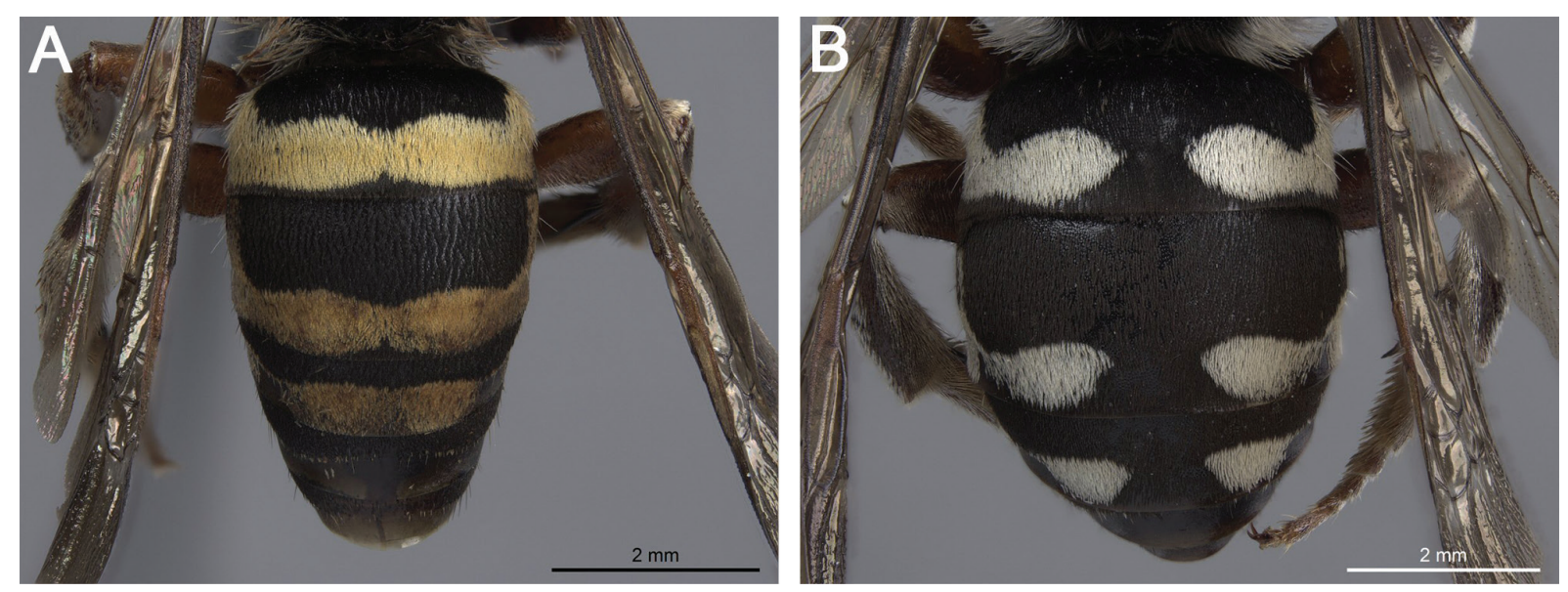

Fig. 9. Metasoma of male, dorsal view. A. Brachymelecta haitensis (Michener, 1948) (JAG). B. B. tibialis (Fabricius, 1793) (UPRM). 
head, mesosoma, and metasoma are pale yellow. Brachymelecta haitensis is the only species in the genus known to occur in Hispaniola, where it is endemic.

\section{Material examined}

\section{Holotype}

HAITI • ̊̊; Ouest, Port-au-Prince; G.N. Wolcott leg.; USNM 534228.

\section{DNA barcoded material}

Available. BIN: BOLD:AEH8436. Specimen examined and sequenced:

DOMINICAN REPUBLIC • 1 §̊; Indepencencia, Sierra de Bahoruco, Caseta \#2; 23 Nov. 2008; J.A. Genaro leg.; BOLD sample ID: CMNTO_037; JAG.

\section{Non-barcoded material}

DOMINICAN REPUBLIC • 2 ふえ‡; Indepencencia, La Descubierta, Lago Enriquillo, Sierra de Neiba; Nov. 2006; J.A. Genaro leg.; JAG • 1 क, 1 ô; same collection data as for preceding; PCYU • 1 q; La Vega, Los Tablones, La Laguna, Parque Nacional José Armando Bermúdez; 30 Jun. 2004; D. Pérez leg.; USNM RD-251.
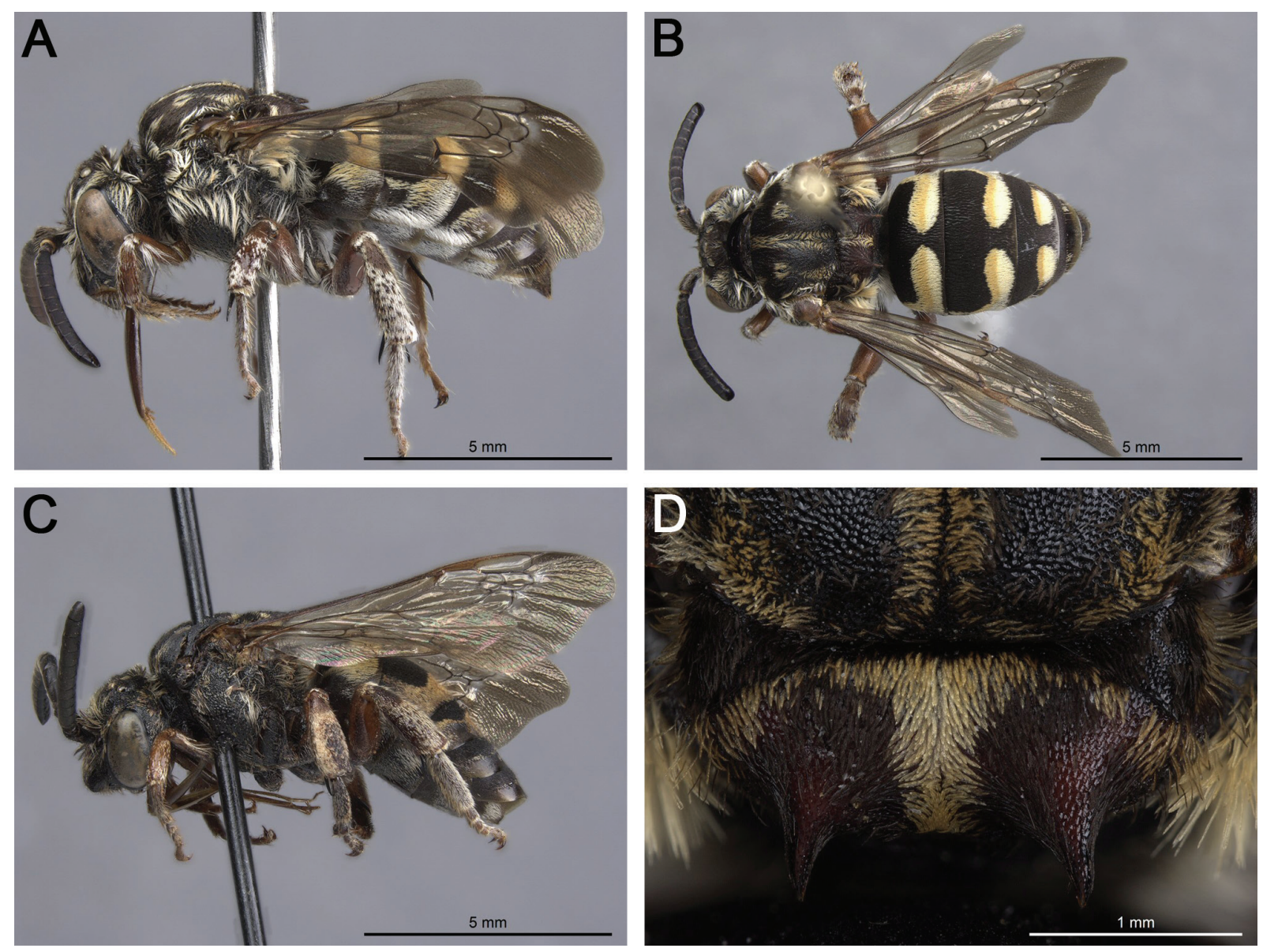

Fig. 10. Brachymelecta haitensis (Michener, 1948). A-B. Female (USNM RD-251). A Habitus, lateral view. B Habitus, dorsal view. C. Male (JAG), habitus, lateral view. D. Female (PCYU), axillae and mesoscutellum, dorsal view. 


\section{Redescription}

\section{Male}

MEASUREMENTs. Length $10.3 \mathrm{~mm}$; ITW $2.4 \mathrm{~mm}$; head length $2.6 \mathrm{~mm}$; head width $2.9 \mathrm{~mm}$; fore wing length $8.6 \mathrm{~mm}$.

InTEGUMent COLORATIOn. Dark brown to black except as follows. Mandible with apical third reddish brown (golden yellow in some non-type specimens). Mandible with middle third, labrum, mesoscutellum, metanotum, and legs, excluding dark brown coxae and tibial spurs (black in non-type specimens), brownish orange (reddish brown to reddish orange in multiple non-type specimens). Tegula amber. Fore wing dusky subhyaline throughout except around third submarginal crossvein and second recurrent vein, where hyaline (all wings badly damaged/stained in holotype; described from non-type specimens). Hind wing dusky subhyaline to hyaline. Much of antenna and metasoma reddish brown in holotype, possibly due to discoloration/fading; dark brown to black in non-type specimens.

PUBESCENCE. Badly matted in holotype (apparently due to prior wetting); described from non-type specimens. Face with hairs densest around antennal socket, predominantly off-white/pale yellow but dark brown/gray above antennal socket and in lower paraocular area. Clypeus with upper half densely hairy; lower half nearly bare. Upper paraocular and frontal areas and vertexal area (except along preoccipital ridge and margin of compound eye) mostly exposed. Head with dense, erect hairs along preoccipital ridge off-white/pale yellow except for pair of dark brown/gray patches, each behind lateral ocellus, separated by small band of off-white/pale yellow hairs along midline between median ocellus and preoccipital ridge, seemingly continuous with band of pale hairs along midline of mesoscutum. Genal beard hairs predominantly off-white/pale yellow. Mesoscutum sparsely hairy except densely hairy anteriorly and along margins, with hairs short, appressed, and predominantly dark brown/gray except for small patch of pale yellow hairs on each side along anterior margin between midline and pronotal lobe, pair of central spots of pale yellow hairs and narrow band extending posteriorly from each toward axilla, well-defined band of pale yellow hairs along midline, and pale yellow hairs along margins. Axilla with conspicuous patch of black hairs. Mesoscutellum with well-defined band of pale yellow hairs along midline, seemingly continuous with band of pale hairs along midline of mesoscutum, and small patch of pale yellow hairs laterally but with pubescence otherwise dark brown or black and sparse, not obscuring underlying integument. Metanotum with short, appressed off-white to dark
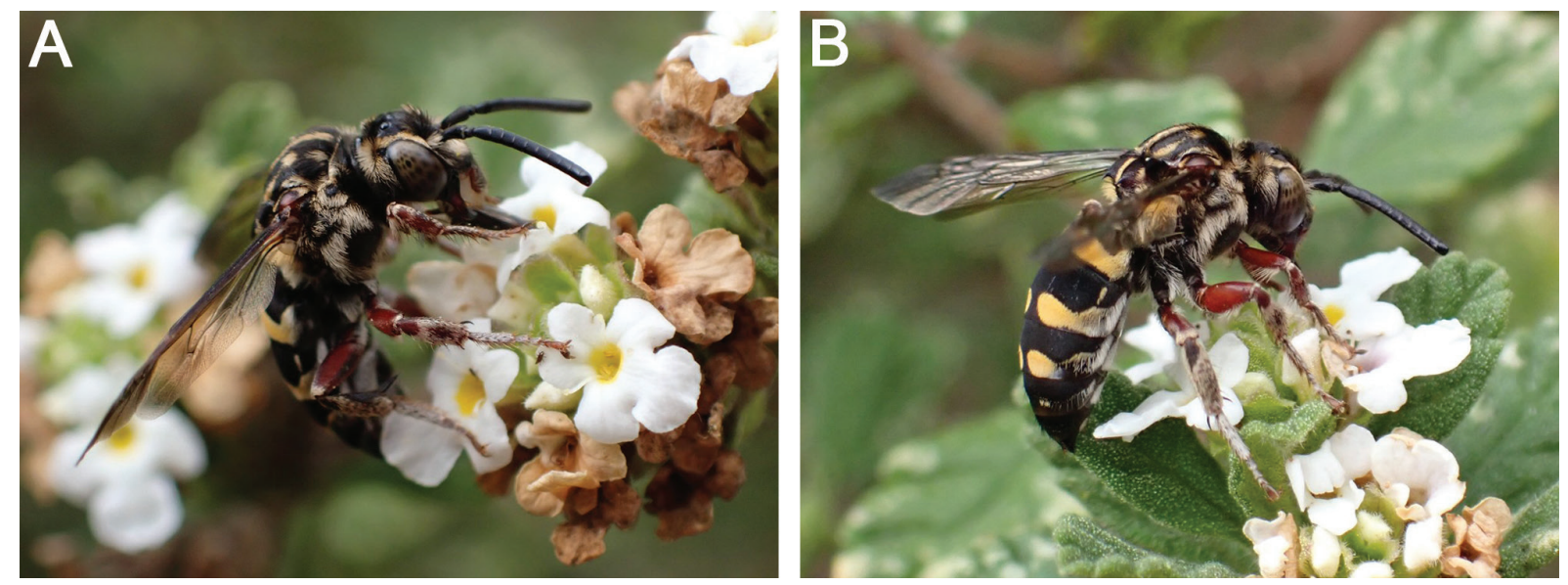

Fig. 11. Female Brachymelecta haitensis (Michener, 1948) taking nectar from flowers of Mexican oregano (Lippia graveolens Kunth (Verbenaceae)) (location: Altamira, Puerto Plata, Dominican Republic). A. Shown with mouthparts extended into corolla. B. Shown with mesoscutellum and metasomal fasciae in view. Images courtesy of L. McDowell Johnson. 
brown hairs. Propodeum with erect, predominantly pale yellow hairs. Mesopleuron sparsely hairy, but with (off-white/pale yellow) hairs moderately dense ventrally as well as between two sparsely hairy circular patches (one beneath base of fore wing (hypoepimeral area), a larger one occupying much of ventrolateral half of mesopleuron). Legs, from coxae to tarsi, with appressed and erect off-white to pale yellow hairs, ventrally with golden-yellow hairs. Profemur with posteroventral fringe of dense, off-white hairs. Protibia with two bands or spots of short, appressed off-white hairs, one in basal half and one on apical margin. Mesotibia with patch of very dense short, appressed off-white/pale yellow hairs, occupying nearly entire dorsal surface. Metatibia with patch of sparser off-white/pale yellow hairs in apical two-thirds. T1-T3 with well-defined complete apical fasciae, those of T1 and T3 each with lobe-like anterolateral extension on each side with erect among appressed pale yellow hairs, that of T2 with pair of anterolateral extensions on each side. T3 with fascia laterally removed from apical margin, narrowed or interrupted mesad each anterolateral extension. T4-T6 without fasciae, although T4 with few sparsely scattered pale hairs present on apical impressed area. Exposed metasomal sterna mainly with short, appressed off-white hairs.

SURFACE SCULPTURE. Labrum and clypeus with punctures equally dense (most $\mathrm{i} \leq 1 \mathrm{~d}$ ). Integument lateral to lateral ocellus punctate. Mesoscutum and mesoscutellum with fine punctures, not much coarser than those of metasomal terga. Mesoscutum and mesoscutellum with punctures equally dense (most $\mathrm{i}<1 \mathrm{~d}$ ) and similar in size. Mesopleuron with denser $($ most $\mathrm{i}<1 \mathrm{~d})$ punctures in upper half than ventrolateral half (many $\mathrm{i}=1-2 \mathrm{~d}$ ), interspaces well-defined and somewhat dull due to tessellate surface microsculpture. Discs of metasomal terga with punctures very fine, dense (i $\approx 1 \mathrm{~d}$ ), interspaces dull due to tessellate surface microsculpture (difficult to see in holotype because integument obscured by badly matted pubescence; described from non-type specimens).

STRUCTURE. Mandible tridentate, with small inner tooth approximately $1 / 3$ length of mandible from base and slightly larger inner tooth approximately $1 / 3$ length of mandible from apex as well as usual large apical tooth (rutellum). Maxillary palpus apparently with one palpomere (three according to Michener 1948). Scape with greatest length $1.8 \times$ greatest width. F2 nearly as long as wide (L/W ratio $=0.9$ ). Mesoscutellum strongly bigibbous, with pair of long, acute, subparallel spines, directed posteriorly. Lateral surface of propodeum posterior to spiracle with rugose crescent ridge, strongly carinate above and joining anterior lip of spiracle. Fore wing with three submarginal cells. T7 with slight median emargination.

\section{Female}

Description as for male except for usual secondary sexual characters and as follows: scape longer, with greatest length $2.1 \times$ greatest width; mesotibia dorsally with large glabrous area between submedial band of off-white to pale yellow hairs (nearer base than apex) and band on apical margin; T1-T3 with fasciae narrowly interrupted medially; T4 in some specimens with pair of small spots of pale yellow hairs along anterior margin of apical impressed area; T6 with narrow, V-shaped but apically rounded pygidial plate with median longitudinal ridge.

\section{Distribution}

Previously known only from Haiti and herein newly reported from the Dominican Republic, this is the only species of Brachymelecta known to occur on the island of Hispaniola (Fig. 4A).

\section{Ecology}

\section{Host records}

Unknown. Given that New World Melectini have been associated only with anthophorine bees (mostly Anthophora), presumably B. haitensis is a cleptoparasite of one or more of the three species 
of Anthophora - A. hilaris, A. hispaniola Brooks, 1999, and A. tricolor (Fabricius, 1775) - known to occur in Hispaniola (see Brooks 1999).

\section{Floral records}

Lippia graveolens Kunth (Verbenaceae) (Fig. 11). Images on iNaturalist (https://www.inaturalist.org) show this species visiting Berylsimpsonia B.L.Turner (Asteraceae) (det. by J. Llamacho). One of the authors of the present study (JAG) has observed males of this species visiting the flowers of Lantana.

\section{Remarks}

Michener (2007) indicated that in the Antillean species of Xeromelecta the maxillary palpi have three (or possibly sometimes two) palpomeres. Both B. alayoi and B. haitensis were originally described as having three-segmented maxillary palpi. In the original description of the latter species, Michener (1948) indicated that in the only other Antillean species known at that time (M. pantalon, later determined to be a junior synonym of $X$. tibialis) the maxillary palpi were reportedly two-segmented but noted that the discrepancy was possibly the result of an observational error by Dewitz (1881) as the palpi are minute. In the present study, the maxillary palpus of a male specimen of $B$. haitensis (BOLD sample ID: CMNTO_037; JAG) was visualized with an SEM, which revealed an enlarged basal portion but no discernible articulations to suggest it has three or even two palpomeres (see Fig. 12). The maxillary palpi of two other specimens (the holotype and a non-type female) were also examined, albeit under a dissecting microscope, and similarly in each an enlarged basal portion was clearly evident but separate palpomeres were not.

Brachymelecta haitensis exhibits unusual sexual dimorphism in that in females the T1-T3 apical fasciae are interrupted medially, albeit narrowly, whereas in males the T1-T3 apical fasciae are complete. This difference between the sexes is consistent across the specimens from Hispaniola that were studied. Previously known from a single (male) specimen, the female of B. haitensis is described here for the first time.
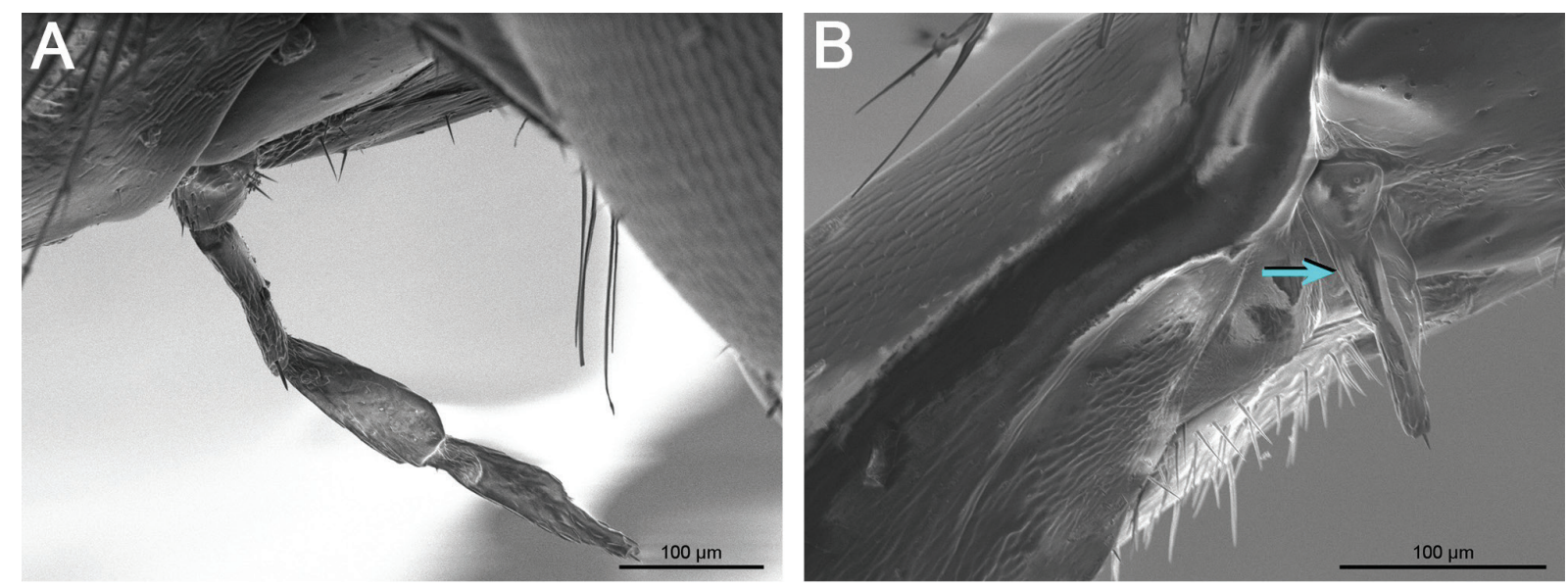

Fig. 12. SEM images of maxillary palpus, lateral view, of male. A. Brachymelecta californica (Cresson, 1878) (BOLD sample ID: CCDB-34570 A05; PCYU), which distinctly has four palpomeres. B. B. haitensis (Michener, 1948) (BOLD sample ID: CMNTO_037; JAG), which has no distinct articulations beyond its attachment to the rest of the maxilla and thus one palpomere (blue arrow). 


\section{Brachymelecta interrupta (Cresson, 1872)}

Figs 4C, 6D, 13, 14B, 17

Melecta interrupta Cresson, 1872: 275 (†, đ); Cresson, 1916: 121 (†) (lectotype designation). Melecta interrupta var. fallugice Cockerell, 1904: 23 (+).

Melecta interrupta var. rociadensis Cockerell, 1904: 23 (ふ).

\section{Proposed common name}

Interrupted digger-cuckoo bee.

\section{Diagnosis}

The following morphological features in combination can be used to tell B. interrupta apart from all other Brachymelecta: the mesoscutum has a pair of anterior spots or (paramedian) bands of hairs darker than the surrounding yellow-orange hairs (Fig. 13B); the mesoscutellum has a pair of short, mammiform tubercles (Fig. 13D); the fore wings are infuscate throughout except around the third submarginal crossvein and second recurrent vein, where they are subhyaline (Fig. 13A-C); and T3 and $\mathrm{T} 4$ each have a medially interrupted fascia that is broadened anterolaterally, that of T3 into the inner pair of anterolateral extensions (there are two such lobe-like extensions on each side) (Fig. 13A-C).
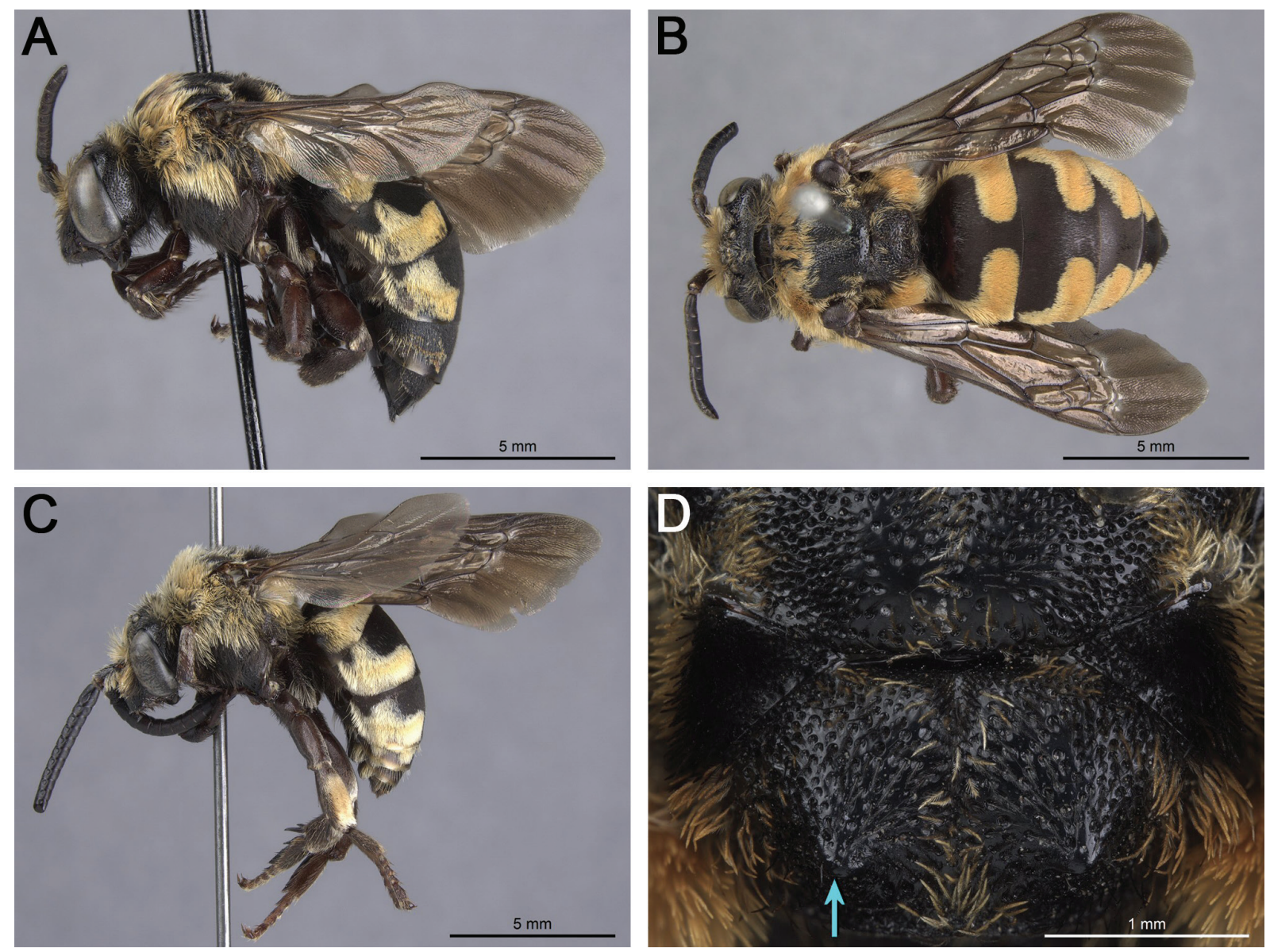

Fig. 13. Brachymelecta interrupta (Cresson, 1872). A. Female (CNC 891735), habitus, lateral view. B. Melecta interrupta lectotype, $q$ (ANSP 2291), habitus, dorsal view. C. Male (CNC 891719), habitus, lateral view. D. Female (BOLD sample ID: 14511H06-AZ; PCYU 0003840), axillae and mesoscutellum, dorsal view (blue arrow indicates one of two short, mammiform tubercles of mesoscutellum). 
Brachymelecta interrupta is most similar to B. californica, but in B. californica the fore wings are infuscate apically, with most cells as well as the membrane around the third submarginal crossvein and second recurrent vein subhyaline, and $\mathrm{T} 3$ and $\mathrm{T} 4$ each have a medially interrupted fascia that is narrowed or interrupted (as opposed to continuously expanded) laterally, mesad the inner pair of anterolateral extensions. Additionally, in B. californica the lighter hairs covering the head, mesosoma, and metasoma are off-white or very rarely pale yellow, whereas in B. interrupta they range from pale yellow to yellow orange.

\section{Material examined}

Primary type material

USA • ${ }^{+}$, M. interrupta var. fallugiae holotype; New Mexico, Pecos; 27 Jun.; M. Grabham leg.; CAS

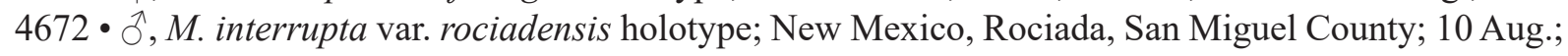
T.D.A. Cockerell leg.; USNM 534231 • + , M. interrupta lectotype; Texas; ANSP 2291.

\section{DNA barcoded material}

Available. BIN: BOLD:ABU5955. Specimens examined and sequenced:

USA 1 1 ; Arizona, Southwestern Research Station (5 mi W of Portal), Cochise County; 28 Aug. 2007; H.T. Ngo leg.; BOLD sample ID: CCDB-34570 B06; PCYU • 1 क; Arizona, Southwestern Research Station (5 mi W of Portal), Cochise County; $31.8840^{\circ}$ N, $109.2060^{\circ}$ W; 31 Aug. 2011; N. de Silva leg.; BOLD sample ID: 14511H06-AZ; PCYU 0003840 • 1 \%; New Mexico, $10 \mathrm{mi}$ N of Mimbres, Grant County; $32.9778^{\circ}$ N, $108.0528^{\circ}$ W; 6 Aug. 2007; M. Buck leg.; BOLD sample ID: CCDB-34570 B04; DEBU debu00291180.

\section{Non-barcoded material}

USA • 1 \&; Arizona, Canelo, Santa Cruz County; 27 Jun. 1965; R.F. Sternitzky leg.; CNC 891717 • 1 §;; same collection data as for preceding; CNC 891718 • 1 O; Arizona, Southwestern Research Station (5 mi

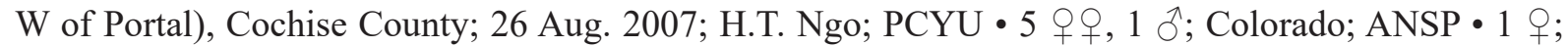
Colorado, Logan County; $40.4894^{\circ} \mathrm{N}, 102.7412^{\circ} \mathrm{W} ; 31$ May 2012; M. Vandever et al. leg.; CSUC • 1 ○;

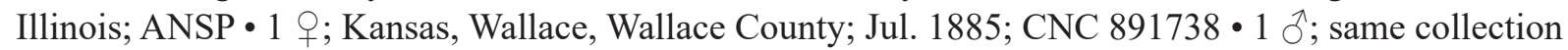
data as for preceding; CNC 891737 • 1 क; Mississippi, Lafayette County; Sep.-Oct.1956; E.R. Wall leg.; CNC 891699 • 1 o; Missouri, Lake Ozark; 20 Sep. 1939; E.C. Van Dyke leg.; CAS • 2 q, 2 ổ; Nebraska; ANSP • 1 \%; New Mexico, 1 mi E of top of pass, near Sierra Blanca, Hwy 70; 27 Aug. 1962; F.M. Hull leg.; CNC 891735 • 1 \%; New Mexico, Manzano Mountains ( 8 mi W of Manzano, near New Canyon Campground), Torrance County; 28 Aug. 1993; J.E. O'Hara leg.; CNC $891714 \bullet 1$ ( (labelled as "Melecta interrupta Cress. O TYPE" but probably a non-type specimen); Texas; USNM 534230 • 1 Jै; Texas, The Rockpile (30 mi NW of Fort Davis); 28 May 1959; J.F. McAlpine leg.; CNC 891719.

\section{Redescription}

\section{Female}

Measurements. Length $12.7 \mathrm{~mm}$; ITW $2.9 \mathrm{~mm}$; head length $3.4 \mathrm{~mm}$; head width $4.0 \mathrm{~mm}$; fore wing length $11.7 \mathrm{~mm}$.

InTEGUMent COLORATION. Dark brown to black except as follows. Mandible with basal two-thirds, legs, excluding black tibial spurs, and much of metasoma reddish brown. Fore wing dusky throughout except around third submarginal crossvein and second recurrent vein, where subhyaline. Hind wing dusky subhyaline.

PuBesCENCE. Face with hairs densest around antennal socket, predominantly pale yellow to yellow orange but almost entirely black in lower paraocular area and on clypeus. Upper paraocular and frontal 
areas and vertexal area (except along preoccipital ridge) mostly exposed. Head with dense, erect hairs along preoccipital ridge yellow orange except for pair of black patches, each behind lateral ocellus and in front of paramedian band (hairs somewhat faded in M. interrupta lectotype; described from nontype specimens). Genal beard hairs almost entirely black. Mesoscutum sparsely hairy except densely hairy anteriorly, narrowly along lateral margin, and in space between parapsidal line and axilla, with hairs predominantly yellow orange except for black paramedian band. Axilla with conspicuous patch of black hairs. Mesoscutellum and much of lateral surface of propodeum (except near bases of mesocoxa and metacoxa) with erect, predominantly yellow-orange hairs. Metanotum and propodeal triangle with erect, predominantly black hairs. Mesopleuron and metapleuron each with upper half covered in dense, yellow-orange hairs greatly obscuring underlying integument and lower half covered in sparser, black hairs not obscuring underlying integument. Legs, from coxae to tarsi, with patches of appressed and erect pale yellow to yellow-orange hairs among more numerous dark brown to black hairs. Profemur with posteroventral fringe of dense, predominantly pale yellow hairs. Tibiae each with small, inconspicuous spot of short, appressed pale yellow hairs in basal half (in some non-type specimens, protibia and metatibia each with additional patch of short, appressed pale yellow hairs on apical margin). T1-T5 with well-defined medially interrupted apical fasciae, laterally with erect among appressed yellow-orange hairs. T2 and T3 fasciae each with pair of lobe-like anterolateral extensions on each side. T3-T5 each with fascia broadened anterolaterally, that of T3 into inner pair of anterolateral extensions. Exposed metasomal sterna with short, appressed black hairs.

SURFACE SCULPTURE. Labrum sparsely punctate (many i $>2 \mathrm{~d}$ ). Clypeus with punctures more or less equally dense throughout (most $i \leq 1 \mathrm{~d}$ ) except for small impunctate shiny spot medially on lower margin. Small impunctate shiny spot lateral to lateral ocellus, another behind median ocellus. Mesoscutum and mesoscutellum with coarser punctures than metasomal terga. Mesoscutum densely punctate (most $i \leq 1 \mathrm{~d}$ ) except for small impunctate shiny spot between paramedian band and parapsidal line and along posterior margin around midline, where more sparsely punctate (few $i>1 d$ ). Mesoscutellum with punctures more or less equally dense throughout (most $\mathrm{i} \leq 1 \mathrm{~d}$ ) and similar in size to those of mesoscutum. Mesopleuron densely punctate (most $\mathrm{i}<1 \mathrm{~d}$ ) but with most interspaces well-defined, shining. Discs of metasomal terga with punctures very fine, sparse (most $i>1$ d), interspaces dull due to tessellate surface microsculpture.

STRUCTURE. Mandible tridentate, with small inner tooth approximately $1 / 3$ length of mandible from base and larger inner tooth approximately $2 / 5$ length of mandible from apex as well as usual large apical tooth (rutellum) (difficult to see in M. interrupta lectotype because mandibles closed; described from non-type specimens). Maxillary palpus with five palpomeres (mouthparts not extended in M. interrupta lectotype; described from non-type specimen). Scape with greatest length $2.3 \times$ greatest width. F2 nearly as long as wide $(\mathrm{L} / \mathrm{W}$ ratio $=0.9)$. Mesoscutellum moderately bigibbous, with pair of short, conical mammiform tubercles. Lateral surface of propodeum posterior to spiracle relatively flat and unmodified. Fore wing with three submarginal cells. T6 with narrow, V-shaped but apically rounded pygidial plate with median longitudinal ridge.

\section{Male}

Description as for female except for usual secondary sexual characters and as follows: scape shorter, with greatest length $1.8 \times$ greatest width; F2 shorter, wider than long $(\mathrm{L} / \mathrm{W}$ ratio $=0.7)$; mesotibia with patch of very dense short, appressed yellow-orange hairs, occupying nearly entire dorsal surface; T7 with slight median emargination; S4 and S5 each with apical/subapical fringe of dense curved black hairs.

\section{Distribution}

Brachymelecta interrupta is the second-most widely distributed member of its genus, after B. californica. Its range includes much of the Central United States, parts of the Southwestern United States (as far west as eastern Arizona), and north-central Mexico (Fig. 4C). 


\section{Ecology}

\section{Host records}

According to Mitchell (1962), the host of B. interrupta is Anthophora abrupta, but no details were provided with regard to the basis of this apparent association. That a different species, $A$. walshii Cresson, 1869 , is the primary if not only host of $B$. interrupta is strongly suggested by three separate observations of the latter in the presence/vicinity of the former (by Robertson 1926; Graenicher 1935; Bouseman 1982 ) and there being much greater range overlap (see Ascher \& Pickering 2020) between B. interrupta and $A$. walshii (both are widespread from the Midwestern United States to Western North America) than between $B$. interrupta and A. abrupta (an eastern species).

\section{Floral records}

The holotype of Melecta interrupta var. fallugiae was collected from the flowers of Fallugia Endl. (Rosaceae). Robertson (1929) reported B. interrupta (as M. interrupta) on Coreopsis palmata Nutt. and Pycnanthemum flexuosum (Walter) Britton, Sterns \& Poggenb. (Lamiaceae). Linsley (1939) reported this species on Cleome L. (Cleomaceae), Dalea L. (Fabaceae) (as Petalostemon), and Monarda L. (Lamiaceae), Hurd (1953) on Baccharis L., Hurd et al. (1980) on Helianthus annuus, and Bouseman (1982) on H. pauciflorus Nutt. (as H. rigidus) and Rudbeckia hirta L. (Asteraceae). Discover Life (Ascher \& Pickering 2020) cites the following floral record contributed by the Bee Biology and Systematics Laboratory: Cirsium Mill. Images on BugGuide (https://bugguide.net) show this species visiting Dalea candida Willd. The label of one examined voucher specimen further indicates that this species has been collected from Marrubium vulgare L.

\section{Remarks}

Brachymelecta interrupta was described (as Melecta interrupta) from two specimens (one female and one male), from Dallas and Comal Counties (Texas), although it is not clear from the description or the female specimen's labels which locality corresponds to which specimen. We have not seen the male specimen, deposited in the Museum of Comparative Zoology (MCZ) in Cambridge, MA, USA, but according to Cockerell (1907) it is from Dallas, so presumably the female is from Comal County. Cresson (1916) designated the female specimen, deposited in the ANSP (catalog number: 2291), as the lectotype of Melecta interrupta. Confusingly, there is also a female specimen in the primary type collection of the USNM (catalog number: 534230) bearing the label "Melecta interrupta Cress. 9 TYPE". This specimen (also from Texas) bears another label that says "Type No 1764 U.S.N.M" and is listed as the lectotype of M. interrupta in the Department of Entomology Collections database (https://collections.nmnh.si.edu/search/ento/). It is not clear when or if this designation was published or which of the two females constituted the basis for the original description, but given that Cresson (1916), who described the species, designated the specimen at the ANSP as the lectotype, it is herein regarded as such as well (with the male at the MCZ, labelled as a syntype, recognized as the lectoallotype).

Cockerell (1904) described two "varieties" of M. interrupta (both from New Mexico) on the basis that neither precisely agrees with the "typical form from Texas". In Linsley (1939), M. var. fallugiae appears in the synonymy list for $M$. interrupta, although it is referred to as a variety rather than a synonym of M. interrupta, one in which the pale pubescence is more orange than yellow. Mitchell (1962) apparently accepted this as a junior synonym of $X$. interrupta, and we agree with Mitchell's treatment. In the holotype of M. interrupta var. fallugiae, the pale pubescence is the same yellow-orange color exhibited by the lectotype of $M$. interrupta.

Linsley (1939) regarded the other variety (M. var. rociadensis) as a separate species (M. rociadensis) from M. interrupta; however, later Hurd \& Linsley (1951) indicated that they were unable to reliably distinguish $M$. var. rociadensis from $M$. interrupta and therefore synonymized the former name under the latter. Aside from the paler pubescence exhibited by the $M$. var. rociadensis holotype, its description applies equally well to the lectotype of M. interrupta. Cockerell's (1904) description, rather than 
differentiating the $M$. var. rociadensis holotype from the "typical form" of $M$. interrupta, instead lists the features (the dark tegulae and wings and unique metasomal markings) that separate $M$. var. rociadensis from $M$. miranda (now a junior synonym of $B$. californica). These differences, however, can be used to separate any representatives of $B$. interrupta (including the lectotype of $M$. interrupta) from the holotype of M. miranda. Although the pale hairs in B. interrupta range from pale yellow to yellow orange, the variation is continuous and the differences in hair color do not correspond to any geographic pattern. Hence, we agree with the treatment of Hurd \& Linsley (1951) and do not regard M. var. rociadensis as a valid taxon.

\section{Brachymelecta larreae (Cockerell, 1900)}

Figs 4D, 6E, 14A, 15, 17

Bombomelecta larrece Cockerell, 1900: 361 (q).

Bombomelecta azygos Viereck, 1903: $181($ đ̊).

\section{Proposed common name}

Creosote digger-cuckoo bee.

\section{Diagnosis}

Unique within the genus to $B$. larreae are each of the following morphological features: the mandibles are bidentate, each with an inner preapical tooth as well as the usual apical tooth (rutellum) (Fig. 14A); the mesoscutum does not have any spots or bands of differentiated hairs but is instead covered in dense, uniformly golden-yellow hairs (Fig. 15A-C); the mesoscutellum has no spines or well-defined mammiform tubercles, although the mesoscutellum is bigibbous to some degree (Fig. 15D); in both sexes the mesotibiae have black hairs only (Fig. 15A, C); and T1-T4 lack well-defined fasciae, although ill-defined apical bands of golden-yellow hairs are sometimes present (Fig. 15A-B). Brachymelecta larreae is more likely to be confused with some Melecta spp. than other species of Brachymelecta, but in North American Melecta the inner ramus of each tarsal claw is narrow, pointed, and thus resembles the outer ramus and T1 has long, pale hairs, similar in length to those on the dorsum of the mesosoma, whereas in B. larreae the inner ramus of each tarsal claw of the mid- and hind legs is broad, lobe-like,
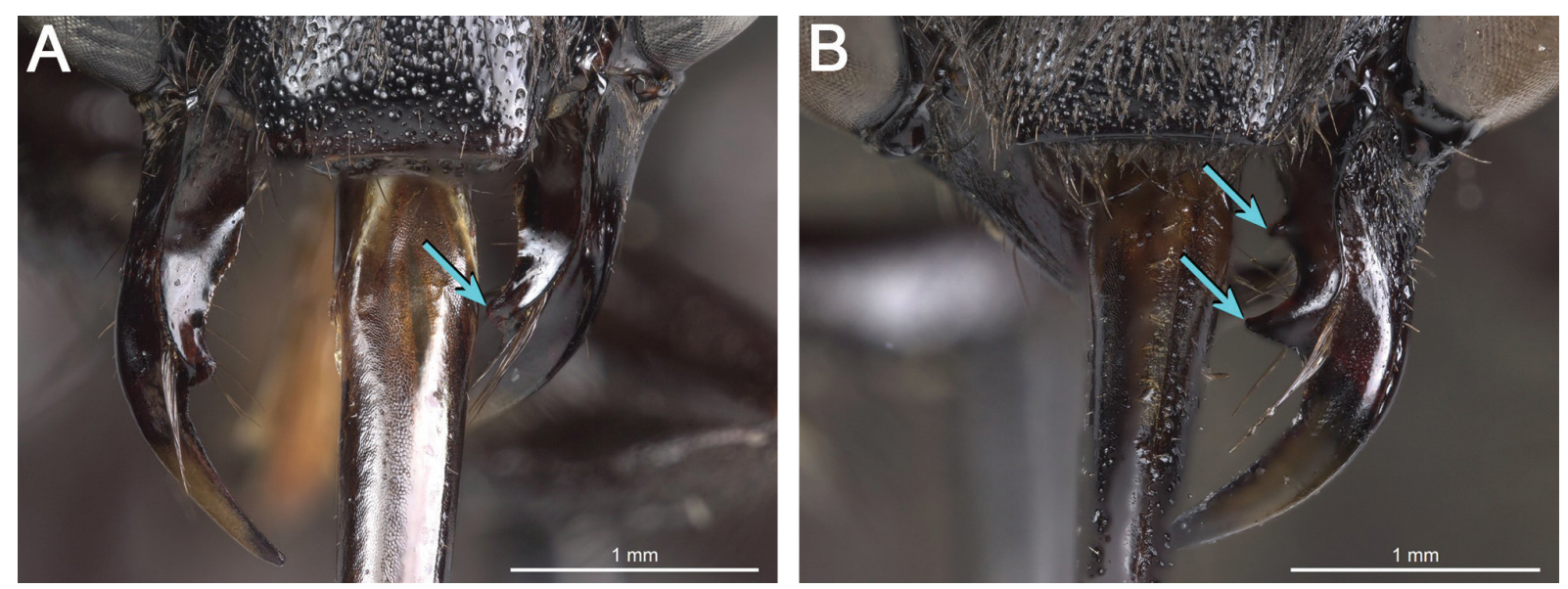

Fig. 14. Mandibles of female. A. Brachymelecta larreae (Cockerell, 1900) (CNC 891712), each mandible has a single inner (preapical/almost submedial) tooth (blue arrow). B. B. interrupta (Cresson, 1872) (CNC 891714), each mandible has both an inner basal tooth as well as an inner preapical (almost submedial) tooth (blue arrows). 
and thus does not resemble the outer ramus (as in all Brachymelecta spp.) and any pale hairs on T1 (the hairs may be entirely black) are distinctly shorter than those on the dorsum of the mesosoma.

\section{Material examined}

Primary type material

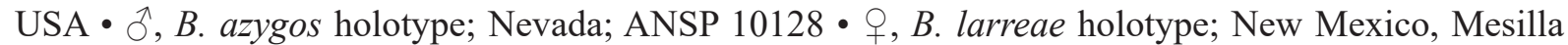
Park, Doña Ana County; 9 May 1900; T.D.A. Cockerell leg.; USNM 534229.

\section{DNA barcoded material}

Available, but not BIN-compliant. Specimen examined and sequenced:

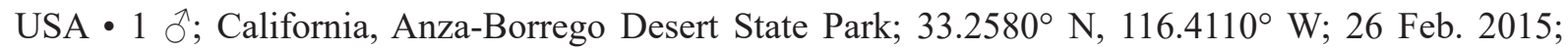
K.J. Hung leg.; BOLD sample ID: CCDB-29918 H07; PCYU SDC.20015.

\section{Non-barcoded material}

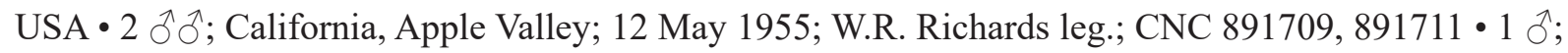
same collection data as for preceding; 20 May 1955; W.R.M. Mason leg.; CNC 899531・3 우, 5 ô California, Argus Mountains, Inyo County; 22 May 1937; E.C. Van Dyke leg.; CAS • 1 ô; California, Helendale, San Bernardino County; 16 May 1955; W.R.M. Mason leg.; CNC 891710 • 4 + $O$; California,
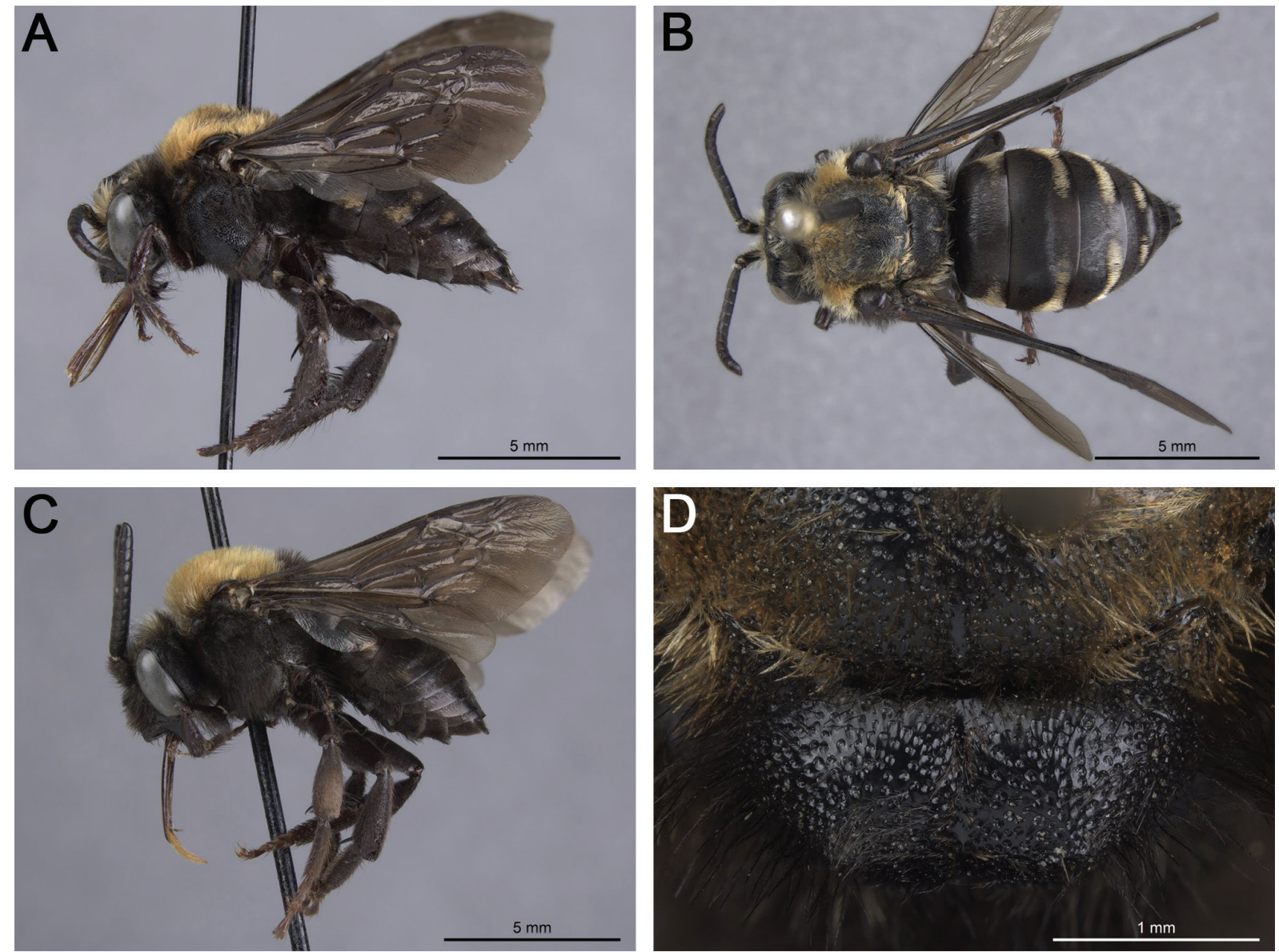

Fig. 15. Brachymelecta larreae (Cockerell, 1900). A, D. Female (CNC 891708). A. Habitus, lateral view. B. Female (CNC 891716), habitus, dorsal view. C. Male (CNC 899531), habitus, lateral view. D. Axillae and mesoscutellum, dorsal view. 
Lone Pine, Inyo County; 24 May 1937; E.C. Van Dyke leg.; CAS • 1 + ; California, Red Mountain; 16 May 1955; J.E.H. Martin leg.; CNC 891712 • 1 +; California, Victorville, San Bernardino County; 20 May 1955; W.R. Richards leg.; CNC 891708 1 क; Texas, Big Bend National Park (near Chilicotal Mountain); 3 May 1959; J.F. McAlpine leg.; CNC 891716.

\section{Redescription}

\section{Female}

Measurements. Length $12.3 \mathrm{~mm}$; ITW $3.3 \mathrm{~mm}$; head length $3.4 \mathrm{~mm}$; head width $3.9 \mathrm{~mm}$; fore wing length $11.0 \mathrm{~mm}$.

INTEGUMENT COLORATION. Dark brown to black except as follows. Mandible with apical third golden yellow. Mandible with basal two-thirds, propodeum, legs, excluding black tibial spurs, and much of metasoma with reddish tinge. Fore wing dusky throughout except around third submarginal crossvein and second recurrent vein, where dusky subhyaline. Hind wing dusky subhyaline.

PubesCence. Face with hairs densest around antennal socket, predominantly pale yellow but more extensively black in lower paraocular area and on clypeus. Upper paraocular and frontal areas and vertexal area (except along preoccipital ridge) mostly exposed. Head with dense, erect hairs along preoccipital ridge entirely black except medially where some hairs pale yellow. Genal beard hairs entirely black. Mesoscutum sparsely hairy except densely hairy anteriorly and along margins, with hairs entirely golden yellow. Axilla dorsally with hairs predominantly golden yellow; laterally with hairs predominantly black. Mesoscutellum, metanotum, and much of propodeum (except behind base of hind wing) with erect, predominantly black hairs; hairs otherwise golden yellow. Mesopleuron (except around pronotal lobe where some hairs pale yellow) and metapleuron each covered in sparse, black hairs not obscuring underlying integument. Legs with dark brown to black hairs only. Profemur with posteroventral fringe of dense hairs. Tibiae sparsely hairy. T1 with apical fascia reduced to pair of small patches of golden-yellow tomentum. T2-T4 with ill-defined apical bands of golden-yellow hairs, darker/interrupted medially. Exposed metasomal sterna with short, appressed black hairs.

SURFACE SCULPTURE. Labrum sparsely punctate (many i $>2 d$ ). Clypeus with punctures more or less equally dense throughout (most $\mathrm{i} \leq 1 \mathrm{~d}$ ). Frontal area below median ocellus with impunctate shiny triangular area. Small impunctate shiny spot lateral to lateral ocellus. Mesoscutum and mesoscutellum with coarser punctures than metasomal terga. Mesoscutum and mesoscutellum with punctures equally dense (most $\mathrm{i} \leq 1 \mathrm{~d})$ and similar in size. Mesopleuron densely punctate (most $\mathrm{i} \leq 1 \mathrm{~d}$ ) but with most interspaces welldefined, shining. Discs of metasomal terga with punctures very fine, sparse (most $i>1 d$ ), interspaces dull due to tessellate surface microsculpture.

STRUCTURE. Mandible bidentate, with small inner tooth approximately $2 / 5$ length of mandible from apex as well as usual large apical tooth (rutellum). Maxillary palpus with five palpomeres. Scape with greatest length $2.3 \times$ greatest width. F2 nearly as long as wide $(\mathrm{L} / \mathrm{W}$ ratio $=0.9)$. Mesoscutellum moderately bigibbous. Lateral surface of propodeum posterior to spiracle relatively flat and unmodified. Fore wing with three submarginal cells. T6 with narrow, V-shaped but apically rounded pygidial plate with median longitudinal ridge.

\section{Male}

Description as for female except for usual secondary sexual characters and as follows: head with black hairs only; scape shorter, with greatest length $1.9 \times$ greatest width; F2 shorter, wider than long (L/W ratio $=0.7)$; mesotibia with patch of very dense short, appressed dark brown to black hairs, occupying nearly entire dorsal surface; T7 with slight median emargination; S4 and S5 each with apical/subapical fringe of dense curved black hairs. 


\section{Distribution}

Brachymelecta larreae is found in the Chihuahuan, Mojave, and Sonoran deserts of North America (Fig. 4D).

\section{Ecology}

\section{Host records}

\section{Unknown.}

\section{Floral records}

The holotype of Bombomelecta larreae was collected from the flowers of Larrea tridentata (Sessé \& Moc. ex DC.) Coville (Zygophyllaceae). Linsley (1939) reported this species on Eriodictyon Benth., Ericameria Nutt. (as Stenotopsis), and Stanleya Nutt. Floral records from data contributors to Discover Life (Ascher \& Pickering 2020) compiled by J. Pickering are as follows: Dalea, Encelia actonii Elmer, Eriodictyon trichocalyx A. Heller, Eriogonum fasciculatum Benth., and Hyptis Jacq. (Lamiaceae). The label of one examined voucher specimen further indicates that this species has been collected from Hyptis emoryi Torr.

\section{Remarks}

Linsley (1938) identified the holotype of Bombomelecta azygos as the male of B. larreae and synonymized the former name under the latter. The holotypes of both B. azygos and B. larreae were examined in the present study, and we agree with Linsley's treatment.

This species exhibits unusual sexual dimorphism in that in females many if not most hairs around the antennal sockets and behind the ocelli are pale yellow, whereas in males the hairs on the head are entirely black. Although in this species the mesoscutellum can be entirely mutic (i.e. without tubercles), in some specimens of $B$. larreae the mesoscutellum has a pair of very small and thus ill-defined tubercles. Based on known records, adults of $B$. larreae are active from late February to early June.

Although BIN-compliant sequences are presently not available for $B$. larreae, a partial sequence, $338 \mathrm{bp}$ in length, is available for a male specimen (BOLD sample ID: CCDB-29918 H07), which was included in the matrix used to construct a phylogeny for Brachymelecta (vide infra).

\section{Brachymelecta tibialis (Fabricius, 1793)}

Figs 4A, 9B, 16-17

Nomada tibialis Fabricius, 1793: $346($ đ).

Crocisa pantalon Dewitz, 1881: 198 (ふ) ), pl. 5, fig. 2.

\section{Proposed common name}

Puerto Rican digger-cuckoo bee.

\section{Diagnosis}

The following morphological features in combination can be used to tell B. tibialis apart from all other Brachymelecta except $B$. haitensis, a Hispaniolan species: the mesoscutum and mesoscutellum both have well-defined bands of pale hairs along the entire midline that connect and thus give the appearance of a single band (Fig. 16B, D); the mesoscutellum sometimes also has pale hairs laterally but the pubescence is otherwise dark brown or black, sparse, and does not obscure the underlying integument (Fig. 16D); and each mesotibia of the female dorsally has a large glabrous area between a submedial band of offwhite hairs (nearer the base than apex) and a band on the apical margin (Fig. 16A). Brachymelecta 
tibialis may be separated from $B$. haitensis as follows. Whereas in B. haitensis the T1-T3 apical fasciae are complete (in males) or only narrowly interrupted medially (in females) and the lighter hairs covering the head, mesosoma, and metasoma are pale yellow, in B. tibialis the T1-T3 apical fasciae are narrowly interrupted medially (in males) or widely interrupted medially (in females) and the lighter hairs covering the head, mesosoma, and metasoma are off-white. Brachymelecta tibialis is the easternmost species in its genus and the only one known to occur in Puerto Rico and the U.S. Virgin Islands.

\section{Material examined}

Primary type material

COUNTRY UNKNOWN・ O, N. tibialis holotype; Sehested leg.; NHMD ZMUC 00241576.

PUERTO RICO • $\widehat{\lambda}$, C. pantalon holotype (studied from images: http://coll.mfn-berlin.de/u/c2e879); Krug leg.; ZMB 22477 for specimen, 22592 for dissected mouthparts.

\section{DNA barcoded material}

Unavailable.
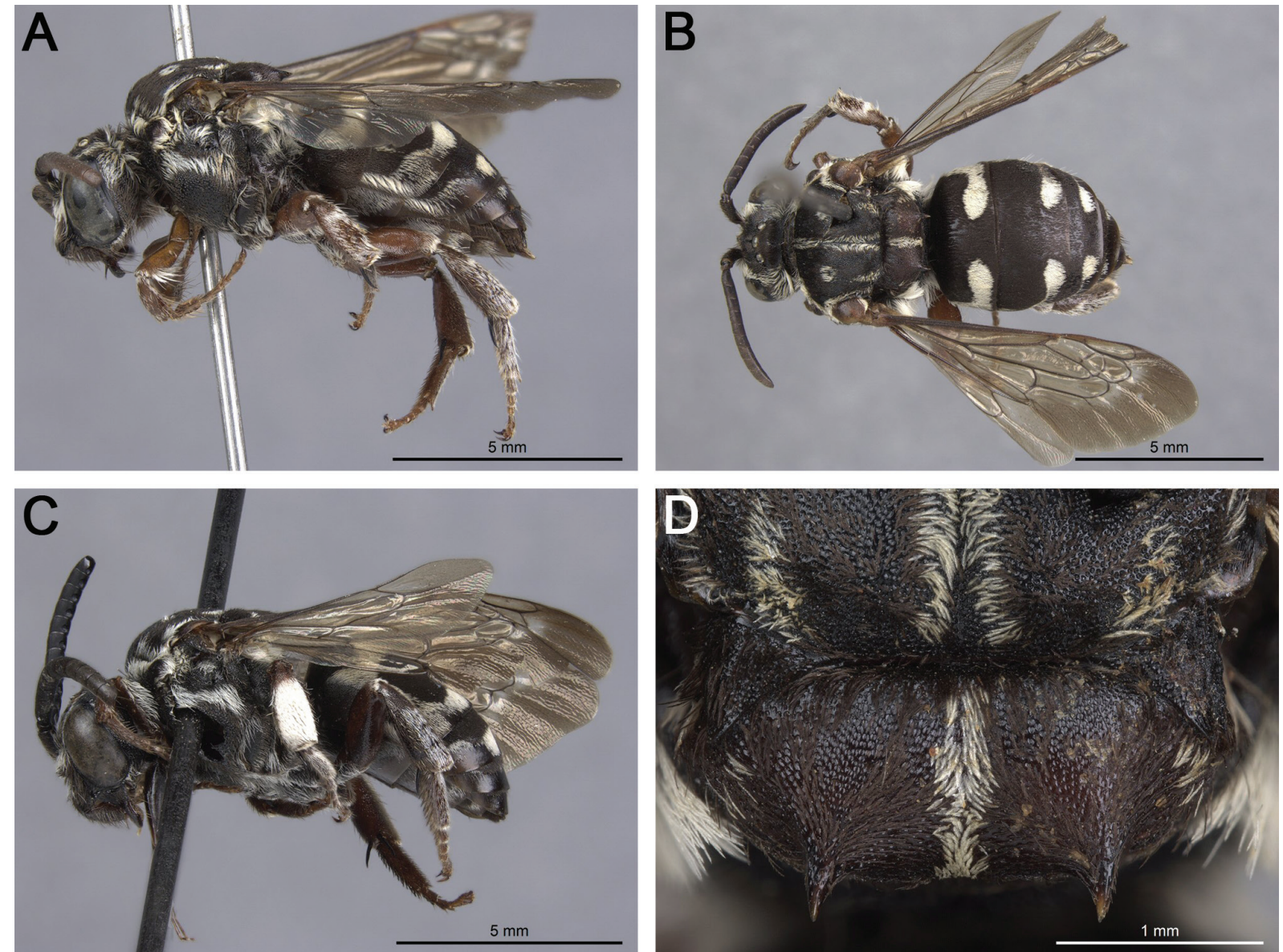

Fig. 16. Brachymelecta tibialis (Fabricius, 1793). A-B, D. Female (INHS 427051). A. Habitus, lateral view. B. Habitus, dorsal view. C. Male (UPRM), habitus, lateral view. D. Axillae and mesoscutellum, dorsal view. 


\section{Non-barcoded material COUNTRY UNKNOWN・1 §’; “American Isles”; Beck leg.; NHMD.}

PUERTO RICO • 1 क; Aguada, Cerro Gordo; 23 Aug. 1987; Douglas II leg.; UPRM・ 1 đ; Lares, Lares; 3 Nov. 1922; F. Sein leg.; UPRM Acc. 416-1922 • 1 ơ; Orocovis, Orocovis; 3 Oct. 1979; A.E.Q. leg.; UPRM・ 1 क; Utuado, Utuado; Jul. 1941; G. Lamboglia leg.; INHS 427051.

UNITED STATES VIRGIN ISLANDS • 1 ô; St. Croix; Eggers leg.; NHMD.

\section{Redescription}

\section{Male}

Measurements. Length $9.4 \mathrm{~mm}$; ITW $2.6 \mathrm{~mm}$; head length $2.7 \mathrm{~mm}$; head width $3.0 \mathrm{~mm}$; fore wing length $8.7 \mathrm{~mm}$.

InTEGUMent COLORATION. Dark brown to black except as follows. Mandible with apical third golden yellow. Mandible with middle third, labrum, mesoscutellum, metanotum, and legs, excluding dark brown to black coxae and tibial spurs, reddish orange (reddish brown in some non-type specimens). Tegula amber. Fore wing dusky subhyaline throughout except along posterior margin and around third submarginal crossvein and second recurrent vein, where hyaline. Hind wing dusky subhyaline to hyaline. Much of metasoma with reddish tinge.

PuBESCENCE. Face with hairs densest around antennal socket, predominantly off-white/pale yellow but dark brown/gray above antennal socket and in lower paraocular area. Clypeus with upper half densely hairy; lower half nearly bare. Upper paraocular and frontal areas and vertexal area (except along preoccipital ridge and margin of compound eye) mostly exposed. Head with dense, erect hairs along preoccipital ridge off-white except for medial dark brown/gray patch behind ocelli. Genal beard hairs predominantly dark brown/gray. Mesoscutum sparsely hairy except densely hairy anteriorly and along margins, with hairs short, appressed, and predominantly dark brown/gray except for small patch of offwhite hairs on each side along anterior margin between midline and pronotal lobe, pair of central spots of off-white hairs, small patch of off-white hairs in front of axilla, well-defined band of off-white hairs along midline, and off-white hairs along margins. Axilla with conspicuous patch of black hairs. Mesoscutellum with well-defined band of off-white hairs along midline, seemingly continuous with band of pale hairs along midline of mesoscutum, and small patch of off-white hairs laterally but with pubescence otherwise dark brown or black and sparse, not obscuring underlying integument. Metanotum with short, appressed dark brown hairs. Propodeum with erect, predominantly off-white hairs. Mesopleuron sparsely hairy, but with (off-white) hairs moderately dense ventrally as well as between two sparsely hairy circular patches (one beneath base of fore wing around somewhat densely hairy hypoepimeral area, a larger one occupying much of ventrolateral half of mesopleuron). Legs, from coxae to tarsi, with appressed and erect off-white hairs, ventrally with golden-yellow hairs. Profemur with posteroventral fringe of dense, off-white hairs. Protibia with two bands or spots of short, appressed off-white hairs, one in basal half and one on apical margin. Mesotibia with patch of very dense short, appressed off-white hairs, occupying nearly entire dorsal surface. Metatibia with patch of sparser off-white hairs in apical two-thirds. T1-T3 with well-defined, medially interrupted apical fasciae, each with lobe-like anterolateral extension on each side with erect among appressed off-white hairs (that of T2 with pair of anterolateral extensions on each side in C. pantalon holotype and some non-type specimens). T3 with fascia laterally removed from apical margin, narrowed or interrupted mesad each anterolateral extension. T4-T6 without fasciae, although T4 with few sparsely scattered pale hairs present on apical impressed area in some non-type specimens. Exposed metasomal sterna mainly with short, appressed off-white hairs. 
SURFACE SCULPTURE. Labrum and clypeus with punctures equally dense (most $i \leq 1 \mathrm{~d}$ ). Small impunctate shiny spot lateral to lateral ocellus. Mesoscutum and mesoscutellum with fine punctures, not much coarser than those of metasomal terga. Mesoscutum and mesoscutellum with punctures equally dense (most $\mathrm{i}<1 \mathrm{~d}$ ) and similar in size. Mesopleuron with denser (most $\mathrm{i}<1 \mathrm{~d}$ ) punctures in upper half than ventrolateral half (many $\mathrm{i}=1-2 \mathrm{~d}$ ), interspaces well-defined and somewhat dull due to tessellate surface microsculpture. Discs of metasomal terga with punctures very fine, dense (i $\approx 1 \mathrm{~d})$, interspaces dull due to tessellate surface microsculpture.

STRUCTURE. Mandible tridentate, with small inner tooth approximately $1 / 3$ length of mandible from base and slightly larger inner tooth approximately $1 / 3$ length of mandible from apex as well as usual large apical tooth (rutellum) (difficult to see in $N$. tibialis holotype because mandibles closed; described from C. pantalon holotype and non-type specimens). Maxillary palpus with one palpomere (mouthparts not extended in $N$. tibialis holotype; described from two non-type specimens). Scape with greatest length $1.8 \times$ greatest width. F2 nearly as long as wide $(\mathrm{L} / \mathrm{W}$ ratio $=0.9)$. Mesoscutellum strongly bigibbous, with pair of long, acute, subparallel spines, directed posteriorly. Lateral surface of propodeum posterior to spiracle with rugose crescent ridge, strongly carinate above and joining anterior lip of spiracle. Fore wing with three submarginal cells. T7 with slight median emargination.

\section{Female}

Description as for male except for usual secondary sexual characters and as follows: scape longer, with greatest length $2.2 \times$ greatest width; mesotibia dorsally with large glabrous area between submedial band of off-white hairs (nearer the base than apex) and band on apical margin; T1-T3 with fasciae more widely interrupted medially; T6 with narrow, V-shaped but apically rounded pygidial plate.

\section{Distribution}

Previously known only from Puerto Rico and herein newly reported from St. Croix, this is the only species of Brachymelecta known to occur on either of the two islands (Fig. 4A).

\section{Ecology}

\section{Host records}

Unknown. Given that New World Melectini have been associated only with anthophorine bees (mostly Anthophora), presumably B. tibialis is a cleptoparasite of $A$. tricolor, the only species in its genus known to occur in Puerto Rico and also the only one represented in the U.S. Virgin Islands (Brooks 1999).

\section{Floral records}

Unknown.

\section{Remarks}

Linsley (1943) described the female of Crocisa pantalon (as Melecta (Melectomorpha) pentalon [sic]) based on a specimen from Lares, Puerto Rico (UPRM Acc. 416-1922), which Genaro \& Franz (2008) determined was actually a male. Although a female specimen was known to Genaro \& Franz (2008), the female of $B$. tibialis is described here for the first time. Donald B. Baker correctly recognized the holotype of Nomada tibialis as belonging to the same species as "Nesomelecta pantalon" (in 1980), and the synonymy was established in Michener (2000) through a personal communication. Moure et al. (2007) erroneously list the USNM and Genaro \& Franz (2008) the NHMD (as ZMUC) as the repository for the holotype of $C$. pantalon. The specimen is actually deposited in the ZMB. 


\section{Key to both sexes of the species of Brachymelecta}

1. Mandible bidentate, with inner preapical tooth as well as usual apical tooth (rutellum) (Fig. 14A). Mesoscutum covered in dense, uniformly golden-yellow hairs (Fig. 15). Mesoscutellum without pair of spines or well-defined mammiform tubercles, although mesoscutellum bigibbous to some degree and sometimes with pair of very minute protrusions (Fig. 15D). Mesotibia with black hairs only (Fig. 15A, C). T1-T4 without fasciae or with only very faint apical bands of golden-yellow hairs (Fig. 15B)

B. larreae (Cockerell, 1900)

- Mandible tridentate, with inner basal tooth as well as inner preapical tooth and usual apical tooth (rutellum) (Fig. 14B). Mesoscutum with pair of spots or bands (Figs 3B, 5B, 10B, 11, 13B, 16B). Mesoscutellum with pair of spines or mammiform tubercles (least developed pair shown in Fig. 13D) (see also Figs 3D, 5D, 10D, 16D). Mesotibia dorsally with some off-white or yellow-orange hairs, those of female sparser and usually reduced to small bands or spots (Figs 3A-B, 5A, 10A-B, $11,16 \mathrm{~A}-\mathrm{B})$; those of male forming patch of very dense off-white, golden-yellow, or yellow-orange hairs, occupying nearly entire dorsal surface (Figs 3C, 5C, 7A, C, 8A-B, 13C, 16C). T1-T3 with well-defined off-white to golden-yellow or yellow-orange apical fasciae (Figs 3A-C, 5A-C, 8A-C, $10 \mathrm{~A}-\mathrm{C}, 11,13 \mathrm{~A}-\mathrm{C}, 16 \mathrm{~A}-\mathrm{C}$ ), rarely entirely covered in pale tomentum (Figs 7A-C, 8D) …........ 2

2. Mesoscutum with pair of anterior spots or bands of hairs darker than surrounding off-white or yellow-orange hairs (Figs 5B, 13B). Mesoscutellum with pair of short, mammiform tubercles (Figs 5D, 13D). Maxillary palpus with four or five palpomeres (Fig. 12A) [North America] .................. 3

- Mesoscutum with pair of central spots of off-white or pale to golden-yellow hairs lighter than surrounding black hairs (Figs 3B, 10B, 11, 16B). Mesoscutellum with pair of spines (Figs 3D, 10D, 16D). Maxillary palpus with fewer than four palpomeres (Fig. 12B) [Greater Antilles]

3. T3 and T4 each with fascia narrowed or interrupted laterally, mesad the inner pair of anterolateral extensions (Fig. 5A-C). Fore wing infuscate apically, subhyaline in most cells and around third submarginal crossvein and second recurrent vein (Fig. 5A-C). Head, mesosoma, and metasoma with pale hairs off-white (rarely pale yellow and never orange) (Figs 5A-C, 8)

B. californica (Cresson, 1878)

- T3 and T4 each with fascia broadened anterolaterally, that of T3 into inner pair of anterolateral extensions (Fig. 13A-C). Fore wing infuscate throughout except subhyaline around third submarginal crossvein and second recurrent vein (Fig. 13A-C). Head, mesosoma, and metasoma with pale hairs typically pale yellow to yellow orange (Fig. 13A-C)

B. interrupta (Cresson, 1872)

4. Mesoscutum with band of pale hairs along midline not attaining posterior margin (Fig. 3B, D). Mesoscutellum, except for pair of spines and around their bases, densely covered in pale pubescence greatly obscuring underlying integument (Fig. 3D) [Cuba]

B. alayoi (Michener, 1988)

- Mesoscutum and mesoscutellum each with uninterrupted band of pale hairs along entire midline (Figs 10B, D, 11, 16B, D). Mesoscutellum pubescence otherwise dark brown or black, sparse, not obscuring underlying integument (Figs 10D, 16D) [other regions]

5. T1-T3 with fasciae pale yellow, narrowly interrupted medially in female (Fig. 10B), complete in male (Fig. 9A) [Hispaniola]

B. haitensis (Michener, 1948)

- T1-T3 with fasciae off-white, widely interrupted medially in female (Fig. 16B), narrowly interrupted medially in male (Fig. 9B) [Puerto Rico and U.S. Virgin Islands] B. tibialis (Fabricius, 1793) 


\section{Phylogeny and biogeography}

Implicit enumeration and extended implied weighting yielded identical topologies. The final tree and associated statistics were generated using implied weights, with $\mathrm{k}$ estimated to be 0.683594 for the morphological partition and 0.390625 for the molecular partition. Maximum parsimony analysis of the combined molecular and morphological dataset produced a fully resolved single most parsimonious tree (length: 627; consistency index: 0.689; retention index: 0.414), in which Brachymelecta was recovered as a monophyletic group sister to Melecta (Fig. 17). Brachymelecta californica was placed as sister to the rest of the genus, rendering the now defunct subgenus Melectomorpha, which included B. californica and B. interrupta, paraphyletic. Brachymelecta interrupta was instead placed as sister to B. larreae, with which it uniquely shares the following morphological features within the genus: the tegulae are dark brown (as opposed to amber), the lateral surface of the propodeum posterior to each spiracle is relatively flat and unmodified (as opposed to having a rugose crescent ridge), and the fore wing membrane in most cells is deeply infuscate (as opposed to subhyaline and contrasting with the infuscate region beyond the veins). The Antillean species (formerly comprising the subgenus Nesomelecta) form a monophyletic group sister to the clade $B$. interrupta $+B$. larreae. The following morphological features exhibited by the Antillean species are not shared with the three other species of Brachymelecta: the maxillary palpus has fewer than four palpomeres; in males, the flagellomeres, excluding F1, are at most as wide as long (as opposed to wider than long); the mesoscutum has a pair of central spots of off-white or pale to golden-yellow hairs lighter than the surrounding dark brown/gray hairs; the mesoscutum is not more conspicuously hairy anteriorly than elsewhere; the mesoscutal and mesoscutellar punctures are not much larger than those on the discs of the metasomal terga; the mesoscutellum has a pair of

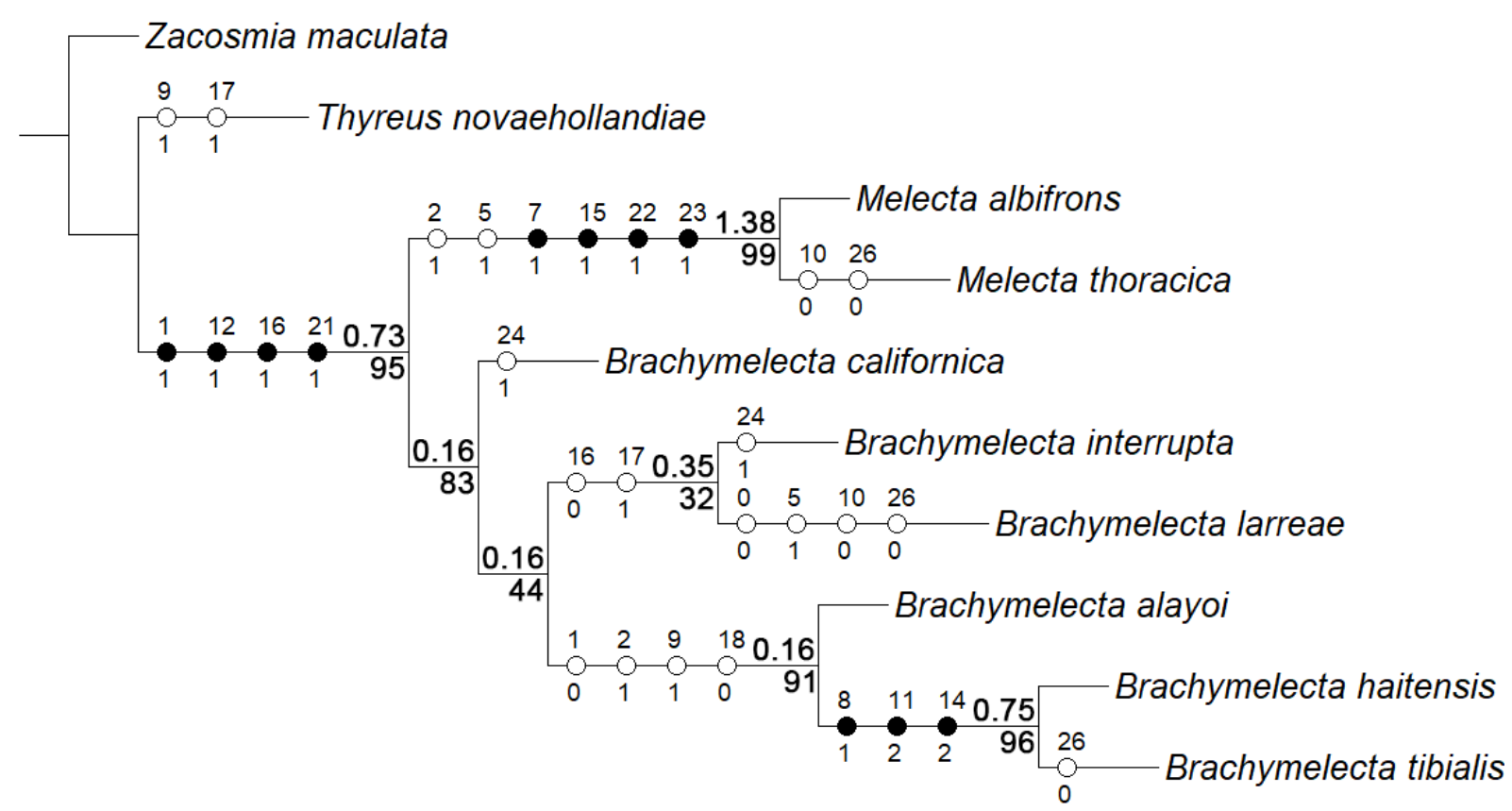

Fig. 17. Fully resolved single most parsimonious tree obtained through maximum parsimony analysis (in TNT) of a combined character set, consisting of 28 morphological characters and DNA barcodes (658 bp, of which 194 were parsimony-informative), with extended implied character weighting. Only morphological characters are shown, with black circles indicating nonhomoplasious changes (autapomorphies or synapomorphies) and empty circles indicating homoplasious changes. Numbers above the circles identify the different characters whereas the numbers below are the assigned character states (see Supp. file 2 for the corresponding descriptions). Numbers to the left of nodes are Bremer support values (above branches) and GC values (below branches). 
long, acute, subparallel spines; the legs are reddish orange and contrast strongly with the dark brown to black mesopleuron; and in males, S4 and S5 both lack a distinct apical/subapical fringe of dense long, curved, suberect hairs. Within this clade, B. haitensis and B. tibialis form a monophyletic group sister to B. alayoi supported by the following morphological features, which are autapomorphic (see Fig. 17): the mesoscutum and mesoscutellum both have well-defined bands of pale hairs along the entire midline and the mesoscutellum and metanotum are reddish brown, contrasting with the predominantly lighter reddish-orange legs.

Within Brachymelecta, three sets of sister nodes were identified as having disjunct distributions when the maximum amount of range overlap was set to the default value of $0 \%$ : the Antillean clade sister to the North American mainland clade that is B. interrupta $+B$. larreae, the Cuban species B. alayoi sister to the clade that is $B$. haitensis $+B$. tibialis, and the Hispaniolan species B. haitensis sister to the Puerto Rican/U.S. Virgin Islands species $B$. tibialis. When the maximum amount of range overlap was increased to $25 \%$, all sister nodes within the genus were identified as having disjunct distributions.

\section{Discussion}

The synonymization of a species of what was the rarest bee genus in North America under a comparatively common species previously regarded to be in a separate genus has some important implications. First, it provides another example of characters that are diagnostic for some higher-level taxa that may vary intraspecifically (or even within an individual) in others. For example, the number of submarginal cells on the fore wing is known to be consistent among the members of some tribes and even subfamilies of bees (Michener 2007), but this feature is also known to be variable within at least 89 species of other bee taxa (reviewed by Scarpulla 2018). This is true for some other genera of cleptoparasitic bees (see Onuferko 2017, 2018 for examples within the nomadine genus Epeolus Latreille, 1802 and Engel et al. 2019 for an example within the nomadine genus Chiasmognathus Engel, 2006). Conversely, some features are often presumed to be unreliable (e.g., integument coloration and patterns of pubescence) because they vary considerably within some species or higher taxa but may be extremely important in diagnosing others. This assumes that the specimens being identified are not worn such that their pubescence has come off or their coloration has faded as a result of prolonged exposure to the elements (before or after capture).

Second, describing new taxa (especially higher-level taxa) from single specimens, although sometimes unavoidable, is undesirable. If unavoidable, such nomenclatural acts should be thoroughly justified, for example, within a phylogenetic context for changes proposed at the supraspecific level, preferably with both molecular and morphological support (e.g., as in Packer et al. 2017). The inherent problem of describing new taxa from single specimens is that observed differences are assumed to be interspecific (and in the case of new genera also intergeneric), since intraspecific (and in the case of new genera also intrageneric) variation is not known (Wiens \& Servedio 2000; Wiens 2007). Thus, in descriptions based on single specimens, there is an increased risk that an aberrant specimen will be misidentified as a new taxon, which could later result in wasted time and resources. According to Engel \& Michener (2012), extensive search efforts have been made (presumably across a large area given the vague type locality) to find additional representatives of Brachymelecta mucida, a species that current evidence suggests does not exist. One of the authors of the present study (LP), for example, searched extensively for the "species" without success at a site in Nevada where Anthophora (Micranthophora) Cockerell, 1906 was abundant; Engel \& Michener (2012) noted that the only North American melectine genus that is as small as B. mucida is Zacosmia, which is a cleptoparasite of A. (Micranthophora) (as A. (Heliophila) Klug, 1807), which is now understood to be a separate subgenus), thereby suggesting that subgenus as a potential host. Moreover, including the present study, there have been four publications in which the taxonomy concerning the holotype of M.? mucida has been assessed/reassessed (Cresson 1879; Linsley 1939; Engel \& Michener 2012), which means that intellectual resources were devoted to a specimen that 
did not merit them. Another consequence resulting from the placement of M.? mucida into its own genus is that all electronic sources in which Xeromelecta is used as a generic name, including encyclopedias, field guides, museum databases, and taxonomic databases, will need to be updated.

Finally, in studies of rare species the type repository/ies should be more carefully searched for additional exemplars of the species of interest. A primary type may be one of a series of conspecific specimens collected at a particular place and time, but primary types are usually kept separate from the main collection. Hence, additional material may be overlooked, especially if it is misplaced and/or identified as something else. It seems probable that the holotype of M.? mucida belongs to the same series of male specimens of B. californica in the ANSP regular collection, with which it shares the same vague locality label (see Fig. 1). Had those specimens not been found, it is unlikely that we would have made a connection between the M.? mucida holotype and the species to which it is now considered to belong, and thus synonymized the genera Brachymelecta and Xeromelecta.

The six species previously placed in Xeromelecta (now Brachymelecta) were found to constitute a natural group. Although no single morphological character state was identified as unique to the genus (see Fig. 17), Brachymelecta is known by a unique combination of morphological features (vide supra) that sets it apart from other bee genera, including its presumed sister genus Melecta. In the dated molecular phylogeny with the greatest sampling of melectine genera to date $(\mathrm{N}=5)$ (Cardinal et al. 2010), Melecta and Xeromelecta (now Brachymelecta) are shown to have diverged from one another around 14 million years ago. Based on this estimate, which if true would presumably mean a more recent crown age for Brachymelecta, instances of allopatric speciation within the genus were presumably the result of dispersal over existing barriers rather than vicariance.

The Windward Passage (separating Cuba and Hispaniola for more than 14 million years) and Mona Passage (separating Hispaniola and Puerto Rico for more than 21 million years) represent obvious barriers that predate the estimated age of the genus (see Liebherr 1988; Iturralde-Vinent 2006) and would have enabled Antillean Brachymelecta to speciate in allopatry. The Gulf of Mexico, which separates this clade from its congeners on the mainland, represents another major barrier to gene flow. At present, it is unclear how Brachymelecta came to inhabit the Caribbean. Southern Florida and the Yucatán Peninsula are the closest areas on the mainland to anywhere in the Greater Antilles. However, there are no recent or historical records of Brachymelecta from either peninsula. Regardless of the route by which Brachymelecta dispersed to the Caribbean, the following factors suggest that Cuba was the first island to be colonized by the genus. Cuba is the nearest island that is inhabited by Brachymelecta to mainland North America. Although a land bridge called GAARlandia (GAAR = Greater Antilles + Aves Ridge) (Iturralde-Vinent \& MacPhee 1999) has been identified that would have enabled terrestrial species to colonize the Greater Antilles from South America via a direct land link to Puerto Rico, current estimates indicate that it would have only been available between 35 and 32 million years ago, which means that more recent colonization from South America would have had to occur via the more difficult dispersal method of island hopping (Crews \& Esposito 2020). Moreover, Brachymelecta is not known to occur or have occurred in South America, and the presumed host genus (Anthophora) is not known to occur in the Lesser Antilles from Martinique southward (Brooks 1999). The dispersal route from Cuba to Hispaniola to Puerto Rico has been inferred in the evolutionary history of a wide range of taxa (Liebherr 1988), including terrestrial arthropods (Crews \& Esposito 2020). Interestingly, Brachymelecta and its host genus Anthophora are both absent from Jamaica (Brooks 1999), an island that although considered one of the Greater Antilles has a rather different geologic and unique biogeographic history compared with the other islands (Crews \& Esposito 2020).

Although the ranges of the three species found on the North American mainland all show some degree of overlap in contrast to the Antillean species (see Fig. 4), it seems probable that B. interrupta and 
B. larreae also speciated in allopatry in light of the following considerations. When disjunctions were redefined to allow for as much range overlap as $10 \%$ between sister taxa in VIP (as opposed to the default value of $0 \%$ ), the sister nodes were identified as disjunct. Additionally, whereas $B$. interrupta inhabits a wide range of habitats and is distributed mainly east of the Continental Divide, $B$. larreae is apparently confined to the three major hot desert regions of North America (Linsley 1939; Hurd 1953). A better understanding of the factors that enabled this genus to diversify through space and time requires detailed knowledge of the evolutionary history of its host bees (presumably all members of the genus Anthophora), which is presently incomplete. As well, a comprehensive phylogeny of Melectini is needed to establish the position of Brachymelecta within its tribe and better understand its origins.

\section{Acknowledgements}

We thank Jason Weintraub for permitting us to borrow the "Melecta" types, including that of M.? mucida, the "Bombomelecta" type, as well as a series of non-type specimens deposited in the ANSP for study and imaging, without which this taxonomic reassessment would have been impossible. We also thank the other institutions and curatorial staff listed in the Material and methods for access to/loans of material for study, especially those who made primary type specimens available to us, including Christopher C. Grinter and Dr. Robert Zuparko (CAS), Dr. José Fernández-Triana (CNC), Dr. Seán Brady and Eugenia Okonski (USNM), and Dr. Lars Vilhelmsen (NHMD), who permitted us to study physical specimens, and Stefanie Krause and Lukas Kirschey (ZMB) and Prof. Michael Engel (KUNHM), who graciously supplied us with detailed images. DNA barcoding at the CBG was carried out by a hardworking team responsible for processing the submitted samples. Roger Bull and Anna Ginter are sincerely thanked for access to the CMN Laboratory of Molecular Biodiversity and their help with the DNA barcoding that took place there. Funds for DNA barcoding were generously provided by Robert and Cecily Bradshaw. The version of TNT used for phylogenetic analysis was subsidized and made freely available by the Willi Hennig Society. This study took place while the primary author was in receipt of a Beaty Postdoctoral Fellowship for Species Discovery (available through the CMN), which has been funded by the Ross Beaty family, whom we sincerely thank for their most generous contribution. We thank François Génier for permission to use and help with the imaging system belonging to the CMN insect collection and Paul Hamilton for access to and help with the museum's SEM imaging system. Additional funding for this study was provided by a discovery grant awarded to the second author by the Natural Sciences and Engineering Research Council of Canada. This article was reviewed for the European Journal of Taxonomy by Dr. Michael Orr and an anonymous reviewer, whom we thank for their constructive and thorough reviews. We also appreciate the time and consideration given to this article by topical editor Dr. Gavin Broad. Finally, we thank all collectors (past and present) and individuals who shared their observations via the online platforms BugGuide, Discover Life, and iNaturalist whose data were used in this study.

\section{References}

Arias J.S., Szumik C.A. \& Goloboff P.A. 2011. Spatial analysis of vicariance: a method for using direct geographical information in historical biogeography. Cladistics 27 (6): 617-628.

https://doi.org/10.1111/j.1096-0031.2011.00353.x

Ascher J.S. \& Pickering J. 2020. Discover Life bee species guide and world checklist (Hymenoptera: Apoidea: Anthophila). Available from http://www.discoverlife.org/mp/20q?guide=Apoidea_species [accessed 24 May 2021].

Bivand R. \& Lewin-Koh N. 2018. maptools: Tools for handling spatial objects. R package version 0.9-4. Available from https:/CRAN.R-project.org/package=maptools [accessed 24 May 2021]. 
Bivand R. \& Rundel C. 2018. rgeos: Interface to geometry engine - open source ('GEOS'). R package version 0.4-2. Available from http://CRAN.R-project.org/package=rgeos [accessed 24 May 2021].

Bossert S., Murray E.A., Almeida E.A.B., Brady S.G., Blaimer B.B. \& Danforth B.N. 2019. Combining transcriptomes and ultraconserved elements to illuminate the phylogeny of Apidae. Molecular Phylogenetics and Evolution 130: 121-131. https://doi.org/10.1016/j.ympev.2018.10.012

Bouseman J.K. 1982. Note on the rediscovery of Xeromelecta interrupta (Cresson) in Illinois (Hymenoptera: Apoidea). Journal of the Kansas Entomological Society 55 (2): 349-350.

Bremer K. 1994. Branch support and tree stability. Cladistics 10 (3): 295-304. https://doi.org/10.1111/j.1096-0031.1994.tb00179.x

Brooks R.W. 1999. Bees of the genus Anthophora Latreille 1803 (Hymenoptera Apidae Anthophorini) of the West Indies. Tropical Zoology 12: 105-124. https://doi.org/10.1080/03946975.1999.10539381

Cardinal S., Straka J. \& Danforth B.N. 2010. Comprehensive phylogeny of apid bees reveals the evolutionary origins and antiquity of cleptoparasitism. Proceedings of the National Academy of Sciences 107: 16207-16211. https://doi.org/10.1073/pnas.1006299107

Cockerell T.D.A. 1900. Notes on New Mexico bees. The Canadian Entomologist 32: 361-364.

https://doi.org/10.4039/Ent32361-12

Cockerell T.D.A. 1904. Descriptions and records of bees. Annals and Magazine of Natural History 14: 21-30. https://doi.org/10.1080/03745480409442962

Cockerell T.D.A. 1907. Some bees in the Museum of Comparative Zoology, Harvard University. University of Colorado Studies 5: 35-39.

Cockerell T.D.A. 1910. Descriptions and Records of Bees. - XXIV. Annals and Magazine of Natural History 5: 22-30. Available from https://www.tandfonline.com/doi/abs/10.1080/00222931008692722 [accessed 24 May 2021].

Cresson E.T. 1872. Hymenoptera Texana. Transactions of the American Entomological Society 4: 153292. https://doi.org/10.2307/25076272

Cresson E.T. 1878. Descriptions of new North American Hymenoptera in the collection of the American Entomological Society. Transactions of the American Entomological Society 7: 61-136.

https://doi.org/10.2307/25076368

Cresson E.T. 1879. Descriptions of new North American Hymenoptera in the collection of the American Entomological Society. Transactions of the American Entomological Society 7: 201-214. https://doi.org/10.2307/25076373

Cresson E.T. 1916. The Cresson types of Hymenoptera. Memoirs of the American Entomological Society 1: $1-141$.

Crews S.C. \& Esposito L.A. 2020. Towards a synthesis of the Caribbean biogeography of terrestrial arthropods. BMC Evolutionary Biology 20: 1-27. https://doi.org/10.1186/s12862-019-1576-z

Dewitz H. 1881. Hymenopteren von Portorico. Berliner Entomologische Zeitschrift 25: 197-208. https://doi.org/10.1002/mmnd.18810250206

Engel M.S. \& Michener C.D. 2012. The melectine bee genera Brachymelecta and Sinomelecta (Hymenoptera, Apidae). ZooKeys 244: 1-19. https://doi.org/10.3897/zookeys.244.3979 
Engel M.S., Packer L. \& Martins D.J. 2019. The cleptoparasitic bee genus Chiasmognathus (Hymenoptera: Apidae) in Kenya, with the description of two new species. Journal of East African Natural History 108: 17-36. https://doi.org/10.2982/028.108.0102

Fabricius J.C. 1793. Entomologia systematica emendata et aucta. Secundum classes, ordines, genera, species adjectis synonimis, locis, observationibus, descriptionibus. Vol. II. Christ. Gottl. Proft, Hafniae [= Copenhagen]. https://doi.org/10.5962/bhl.title.122153

Ferrari R.R. 2017. Taxonomic revision of the species of Colletes Latreille, 1802 (Hymenoptera: Colletidae: Colletinae) found in Chile. Zootaxa 4364: 1-137. https://doi.org/10.11646/zootaxa.4364.1.1

Ferrari R.R. 2019. A revision of Colletes Latreille (Hymenoptera: Colletidae: Colletinae) from Brazil, Paraguay and Uruguay. Zootaxa 4606: 1-91. https://doi.org/10.11646/zootaxa.4606.1.1

Fox W.J. 1893. Synopsis of the North American species of Melecta. Entomological News 4: 143-144.

Genaro J.A. \& Franz N.M. 2008. The bees of Greater Puerto Rico (Hymenoptera: Apoidea: Anthophila). Insecta Mundi 0040: 1-24.

Gibbs J. 2009. New species in the Lasioglossum petrellum species group identified through an integrative taxonomic approach. The Canadian Entomologist 141 (4): 371-396. https://doi.org/10.4039/n09-020

Gibbs J. 2010. Revision of the metallic species of Lasioglossum (Dialictus) in Canada (Hymenoptera, Halictidae, Halictini). Zootaxa 2591: 1-382. https://doi.org/10.11646/zootaxa.2591.1.1

Gibbs J. 2011. Revision of the metallic Lasioglossum (Dialictus) of eastern North America (Hymenoptera: Halictidae: Halictini). Zootaxa 3073: 1-216. https://oi.org/10.11646/zootaxa.3073.1.1

Goloboff P.A. 1999. Analyzing large data sets in reasonable times: solutions for composite optima. Cladistics 15: 415-428. https://doi.org/10.1111/j.1096-0031.1999.tb00278.x

Goloboff P.A. 2014. Extended implied weighting. Cladistics 30: 260-272.

https://doi.org/10.1111/cla.12047

Goloboff P.A., Farris J.S., Källersjö M., Oxelman B., Ramírez M.J. \& Szumik C.A. 2005. Improvements to resampling measures of group support. Cladistics 19: 324-332.

https://doi.org/10.1111/j.1096-0031.2003.tb00376.x

Goloboff P.A., Farris J.S. \& Nixon K.C. 2008. TNT, a free program for phylogenetic analysis. Cladistics 24: 774-786. https://doi.org/10.1111/j.1096-0031.2008.00217.x

Graenicher S. 1935. Bee-fauna and vegetation of Wisconsin. Annals of the Entomological Society of America 28: 285-310. https://doi.org/10.1093/aesa/28.2.285

Hajibabaei M., Janzen D.H., Burns J.M., Hallwachs W. \& Hebert P.D.N. 2005. DNA barcodes distinguish species of tropical Lepidoptera. Proceedings of the National Academy of Sciences 103: 968-971.

https://doi.org/10.1073/pnas.0510466103

Hebert P.D.N., Cywinska A., Ball S.L. \& deWaard J.R. 2003a. Biological identifications through DNA barcodes. Proceedings of the Royal Society of London Series B, Biological Sciences 270: 313-321. https://doi.org/10.1098/rspb.2002.2218

Hebert P.D.N., Ratnasingham S. \& deWaard J.R. 2003b. Barcoding animal life: cytochrome c oxidase subunit 1 divergences among closely related species. Proceedings of the Royal Society of London Series B, Biological Sciences 270: S96-S99. https://doi.org/10.1098/rsbl.2003.0025 
Hebert P.D.N., Penton E.H., Burns J.M., Janzen D.H. \& Hallwachs W. 2004. Ten species in one: DNA barcoding reveals cryptic species in the neotropical skipper butterfly Astraptes fulgerator. Proceedings of the National Academy of Sciences 101: 14812-14817. https://doi.org/10.1073/pnas.0406166101

Hicks C.H. 1926. Nesting habits and parasites of certain bees of Boulder County, Colorado. University of Colorado Studies 15: 217-252.

Hijmans R.J. 2018. raster: Geographic data analysis and modeling. R package version 2.8-4. Available from http://CRAN.R-project.org/package=raster [accessed 24 May 2021].

Hurd P.D. Jr. 1953. Notes on the melectine bees of Mexico (Hymenoptera: Anthophoridae). Journal of the Kansas Entomological Society 26 (1): 35-37.

Hurd P.D. Jr. \& Linsley E.G. 1951. The melectine bees of California (Hymenoptera: Anthophoridae). Bulletin of the California Insect Survey 1: 119-140.

Hurd P.D. Jr., LaBerge W.E. \& Linsley E.G. 1980. Principal sunflower bees of North America with emphasis on the Southwestern United States (Hymenoptera: Apoidea). Smithsonian Contributions to Zoology 310: 1-158. https://doi.org/10.5479/si.00810282.310

Iturralde-Vinent M.A. 2006. Meso-Cenozoic Caribbean paleogeography: implications for the historical biogeography of the region. International Geology Review 48: 791-827.

https://doi.org/10.2747/0020-6814.48.9.791

Iturralde-Vinent M. \& MacPhee R.D.E. 1999. Paleogeography of the Caribbean region: implications for Cenozoic biogeography. Bulletin of the American Museum of Natural History 238: 1-95.

Ivanova N.V., deWaard J.R. \& Hebert P.D.N. 2006. An inexpensive, automation-friendly protocol for recovering high-quality DNA. Molecular Ecology Notes 6: 998-1002.

https://doi.org/10.1111/j.1471-8286.2006.01428.x

Kearse M., Moir R., Wilson A., Stones-Havas S., Cheung M., Sturrock S., Buxton S., Cooper A., Markowitz S., Duran C., Thierer T., Ashton B., Meintjes P. \& Drummond A. 2012. Geneious Basic: An integrated and extendable desktop software platform for the organization and analysis of sequence data. Bioinformatics 28: 1647-1649. https://doi.org/10.1093/bioinformatics/bts199

Liebherr J.K. 1988. The Caribbean: fertile ground for zoogeography. In: Liebherr J.K. (ed.) Zoogeography of Caribbean Insects: 1-14. Comstock Publishing Associates, Cornell University Press, Ithaca and London. https://doi.org/10.7591/9781501746017-003

Lieftinck M.A. 1962. Revision of the Indo-Australian species of the genus Thyreus Panzer (= Crocisa Jurine) (Hym., Apoidea, Anthophoridae) Part 3. Oriental and Australian species. Zoologische Verhandelingen 53: 1-212.

Lieftinck M.A. 1980. Prodrome to a monograph of the palaearctic species of the genus Melecta Latreille 1802 (Hymenoptera, Anthophoridae). Tijdschrift voor Entomologie 123: 129-349.

Linsley E.G. 1938. Notes on the synonymy and distribution of some North American bees of the genus Bombomelecta (Hymenoptera). Entomological News 49: 160-163.

Linsley E.G. 1939. A revision of the Nearctic Melectinae (Hymenoptera, Anthophoridae). Annals of the Entomological Society of America 32: 429-468. https://doi.org/10.1093/aesa/32.2.429

Linsley E.G. 1943. The genus Melecta in eastern North America and Porto Rico (Hymenoptera, Anthophoridae). Journal of the New York Entomological Society 51: 225-227. 
Linsley E.G. \& McSwain J.W. 1942. The parasites, predators, and inquiline associates of Anthophora linsleyi. The American Midland Naturalist 27: 402-417. https://doi.org/10.2307/2421008

Mann B.P. 1885. Herbert Knowles Morrison. Psyche 4: 287. https://doi.org/10.1155/1885/65191

Meier R. 2017. Citation of taxonomic publications: the why, when, what and what not. Systematic Entomology 42 (2): 301-304. https://doi.org/10.1111/syen.12215

Michener C.D. 1948. Notes on the American bees of the genus Melecta (Hymenoptera, Apoidea). Proceedings of the Entomological Society of Washington 50: 15-18.

Michener C.D. 1988. The parasitic anthophorid genus Xeromelecta in Cuba (Hymenoptera: Apoidea). Annals of the Entomological Society of America 81: 377-379. https://doi.org/10.1093/aesa/81.3.377

Michener C.D. 2000. The Bees of the World. The Johns Hopkins University Press, Baltimore, MD.

Michener C.D. 2007. The Bees of the World, Second ed. The Johns Hopkins University Press, Baltimore, MD.

Mir Sharifi N., Graham L. \& Packer L. 2019. Fifteen new species of Liphanthus Reed (Hymenoptera: Andrenidae) with two submarginal cells. Zootaxa 4645(1): 1-80.https://doi.org/10.11646/zootaxa.4645.1

Mitchell T.B. 1962. Bees of the eastern United States. Volume II. North Carolina Agricultural Experiment Station Technical Bulletin 152: 1-557.

Monckton S.K. 2016. A revision of Chilicola (Heteroediscelis), a subgenus of xeromelissine bees (Hymenoptera, Colletidae) endemic to Chile: taxonomy, phylogeny, and biogeography, with descriptions of eight new species. ZooKeys 591: 1-144. https://doi.org/10.3897/zookeys.591.7731

Moure J.S., Urban D. \& Melo G.A.R. 2007. Catalogue of Bees (Hymenoptera, Apoidea) in the Neotropical Region. Sociedade Brasileira de Entomologia, Curitiba.

Nixon K.C. 1999. The Parsimony Ratchet, a new method for rapid parsimony analysis. Cladistics 15 : 407-414. https://doi.org/10.1006/clad.1999.0121

Nixon K.C. 2002. WinClada ver. 1.00. 08. Published by the author, Ithaca, NY.

Onuferko T.M. 2017. Cleptoparasitic bees of the genus Epeolus Latreille (Hymenoptera: Apidae) in Canada. Canadian Journal of Arthropod Identification 30: 1-62. https://doi.org/10.3752/cjai.2017.30

Onuferko T.M. 2018. A revision of the cleptoparasitic bee genus Epeolus Latreille for Nearctic species, north of Mexico (Hymenoptera, Apidae). ZooKeys 755: 1-185.

https://doi.org/10.3897/zookeys.755.23939

Onuferko T.M. 2019. A review of the cleptoparasitic bee genus Epeolus Latreille, 1802 (Hymenoptera: Apidae) in the Caribbean, Central America and Mexico. European Journal of Taxonomy 563: 1-69. https://doi.org/10.5852/ejt.2019.563

Onuferko T.M., Bogusch P., Ferrari R.R. \& Packer L. 2019. Phylogeny and biogeography of the cleptoparasitic bee genus Epeolus (Hymenoptera: Apidae) and cophylogenetic analysis with its host bee genus Colletes (Hymenoptera: Colletidae). Molecular Phylogenetics and Evolution 141: 1-14. https://doi.org/10.1016/j.ympev.2019.106603

Orr M.C., Griswold T., Pitts J.P. \& Parker F.D. 2016. A new bee species that excavates sandstone nests. Current Biology 26: R792-R793. https://doi.org/10.1016/j.cub.2016.08.001 
Packer L., Litman J. \& Praz C.J. 2017. Phylogenetic position of a remarkable new fideliine bee from northern Chile (Hymenoptera: Megachilidae). Systematic Entomology 42: 473-488.

https://doi.org/10.1111/syen.12229

Packer L., Monckton S.K., Onuferko T.M. \& Ferrari R.R. 2018. Validating taxonomic identifications in entomological research. Insect Conservation and Diversity 11 (1): 1-12.

https://doi.org/10.1111/icad.12284

Prentice M.A. 1998. The Comparative Morphology and Phylogeny of Apoid Wasps (Hymenoptera: Apoidea). $\mathrm{PhD}$ thesis, University of California, Berkeley, CA.

R Core Team. 2018. R: A language and environment for statistical computing. R Foundation for Statistical Computing, Vienna, Austria. Available from https://www.R-project.org/ [accessed 24 May 2021].

Ratnasingham S. \& Hebert P.D.N. 2007. BOLD: The Barcode of Life Data System (www.barcodinglife. org). Molecular Ecology Notes 7 (3): 355-364. https://doi.org/10.1111/j.1471-8286.2007.01678.x

Ratnasingham S. \& Hebert P.D.N. 2013. A DNA-based registry for all animal species: the Barcode Index Number (BIN) system. PloS One 8 (7): e66213. https://doi.org/10.1371/journal.pone.0066213

Rightmyer M.G. 2008. A review of the cleptoparasitic bee genus Triepeolus (Hymenoptera: Apidae). Part I. Zootaxa 1710: 1-170. https://doi.org/10.11646/zootaxa.1710.1.1

Robertson C. 1926. Phenology of inquiline and nest-making bees. Psyche 33 (4-5): 116-120. https://doi.org/10.1155/1926/95452

Robertson C. 1929. Flowers and Insects. Lists of Visitors of Four Hundred and Fifty-three Flowers. The Science Press Printing Company, Lancaster, PA. https://doi.org/10.5962/bhl.title.11538

Rocha-Filho L.C. \& Packer L. 2015. Revision of the Neotropical subgenera Coelioxys (Platycoelioxys) Mitchell and C. (Rhinocoelioxys) Mitchell (Hymenoptera; Megachilidae) with the description of one new species. Zootaxa 3941: 151-203. https://doi.org/10.11646/zootaxa.3941.2.1

Roig-Alsina A. \& Michener C.D. 1993. Studies of the phylogeny and classification of long-tongued bees (Hymenoptera: Apoidea). The University of Kansas Science Bulletin 55: 124-162.

https://doi.org/10.5962/bhl.part.775

Rozen J.G. Jr. 1991. Evolution of cleptoparasitism in anthophorid bees as revealed by their mode of parasitism and first instars (Hymenoptera: Apoidea). American Museum Novitates 3029: 1-36.

Santos B.F., Payne A., Pickett K.M. \& Carpenter J.M. 2015. Phylogeny and historical biogeography of the paper wasp genus Polistes (Hymenoptera: Vespidae): implications for the overwintering hypothesis of social evolution. Cladistics 31: 535-549. https://doi.org/10.1111/cla.12103

Scarpulla E.J. 2018. Four submarginal cells on a forewing of Melitoma taurea (Say) (Hymenoptera: Apidae), and a summary of known records of atypical and variable numbers of submarginal cells. Insecta Mundi 0667: 1-28.

Statistics Canada. 2015.Provinces/Territories, 2011 Census (cartographic boundary file, gpr_000b11a_e). Available from

https://www12.statcan.gc.ca/census-recensement/2011/geo/bound-limit/bound-limit-2011-eng.cfm [accessed 24 May 2021].

Tamura K. \& Nei M. 1993. Estimation of the number of nucleotide substitutions in the control region of mitochondrial DNA in humans and chimpanzees. Molecular Biology and Evolution 10 (3): 512-526. https://doi.org/10.1093/oxfordjournals.molbev.a040023 
Torchio P.F. \& Trostle G.E. 1986. Biological notes on Anthophora urbana urbana and its parasite, Xeromelecta californica (Hymenoptera: Anthophoridae), including descriptions of late embryogenesis and hatching. Annals of the Entomological Society of America 79: 434-447. https://doi.org/10.1093/aesa/79.3.434

US Census Bureau. 2015. States, 2014 (cartographic boundary file, cb_2014_us_state_500k). Available from www.census.gov/geographies/mapping-files/time-series/geo/carto-boundary-file.2014.html [accessed 24 May 2021].

Viereck H.L. 1903. Classification of bees of the genus Bombomelecta. Transactions of the American Entomological Society 29: 179-182.

Viereck H.L. 1924. Descriptions of two Canadian bees of the genus Melecta. The Canadian Entomologist 56: 15. https://doi.org/10.4039/Ent5615-1

Wiens J.J. 2007. Species delimitation: new approaches for discovering diversity. Systematic Biology 56: 875-878. https://doi.org/10.1080/10635150701748506

Wiens J.J. \& Servedio M.R. 2000. Species delimitation in systematics: inferring diagnostic differences between species. Proceedings of the Royal Society of London Series B, Biological Sciences 267: 631636. https://doi.org/10.1098/rspb.2000.1049

Manuscript received: 22 September 2020

Manuscript accepted: 3 March 2021

Published on: 15 June 2021

Topic editors: Nesrine Akkari \& Gavin Broad

Desk editor: Jeroen Venderickx

Printed versions of all papers are also deposited in the libraries of the institutes that are members of the EJT consortium: Muséum national d'histoire naturelle, Paris, France; Meise Botanic Garden, Belgium; Royal Museum for Central Africa, Tervuren, Belgium; Royal Belgian Institute of Natural Sciences, Brussels, Belgium; Natural History Museum of Denmark, Copenhagen, Denmark; Naturalis Biodiversity Center, Leiden, the Netherlands; Museo Nacional de Ciencias Naturales-CSIC, Madrid, Spain; Real Jardín Botánico de Madrid CSIC, Spain; Zoological Research Museum Alexander Koenig, Bonn, Germany; National Museum, Prague, Czech Republic.

Supp. file 1. A database of records of Melectini Westwood, 1840. Records of Brachymelecta Linsley, 1939 include specimens personally examined by at least one of the authors as well as online and literature records. Included are geographic coordinates associated with collection/observation localities, which were used to construct range maps and in spatial analysis of vicariance in VIP. Records of studied specimens are also provided for species of other melectine genera that were included in phylogenetic analysis. https://doi.org/10.5852/ejt.2021.754.1393.4421

Supp. file 2. An annotated list of morphological characters scored for six ingroup taxa (species of Brachymelecta Linsley, 1939) and four outgroup taxa (species of other melectine genera) from which the matrix presented in Table 1 was constructed. https://doi.org/10.5852/ejt.2021.754.1393.4423

Supp. file 3. Supplementary figures. https://doi.org/10.5852/ejt.2021.754.1393.4427 